PNL--6584-Vol.1

DE89 004462

HANFORD ENVIRONMENTAL. DOSIMETRY UPGRADE PROJECT

GENII - THE HANFORD ENVIRONMENTAL RADIATION DOSIMETRY SOFTWARE SYSTEM

VOLUME 1: Conceptual Representation
B. A. Napier
R. A. Peloquin
D. L. Strenge
J. V. Ramsdell

December 1988

Prepared for

the U.S. Department of Energy

under Contract DE-ACO6-76RLO 1830

Pacific Northwest Laboratory

Richland, Washington 99352 



\section{ABSTRACT}

The Hanford Environmental Dosimetry Upgrade Project was undertaken to incorporate the internal dosimetry models recommended by the International Commission on Radiological Protection (ICRP) in updated versions of the environmental pathway analysis models used at Hanford. The resulting second generation of Hanford environmental dosimetry computer codes is compiled in the Hanford Environmental Dosimetry System (Generation II, or GENII). The purpose of this coupled system of computer codes is to analyze environmental contamination resulting from acute or chronic releases to, or initial contamination of, air, water, or soil. This is accomplished by calculating radiation doses to individuals or populations.

GENII is described in three volumes of documentation. The first volume describes the theoretical considerations of the system. The second volume is a Users' Manual, providing code structure, users' instructions, required system configurations, and QA-related topics. The third volume is a code Maintenance Manual for the user who requires knowledge of code detail. It includes code logic diagrams, global dictionary, worksheets, example hand calculations, and listings of the code and its associated data libraries. 


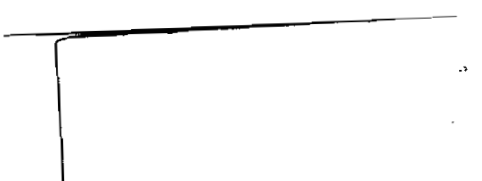




\section{ACKNOWLEDGMENTS}

This work was performed as part of the Dose Overview Project for the U.S. Department of Energy, Richland Operations Office. The authors would like to thank all those individuals who helped make possible the development of this software package. Assisting in developing the project as well as the code package were the successive managers; of the Dose Overview Project: Ron Kathren, the late Ed Watson, Jerry Martin, and R. Gene Schreckhise. Members of the Hanford Dose Overview Pane1, including Darrell Fisher, Paul Rittman, and Janet Davis, provided the initial code capability requirements. Valuable developmental assistance was provided by S. Keith Hargrove, Mike Madison, Jaime Lara, and Fhil Pohl, of the NORCUS REST and GEM programs. We especially thank Joe Soldat for extending his computer literacy as our chief test subject in code debugging. Finally, we would like to thank Peggy Upton, Marianna Cross, and their co-workers for suffering through the editing and production of this series. 

ABSTRACT ............................... $i$... ACKNOWLEDGMENTS ....................

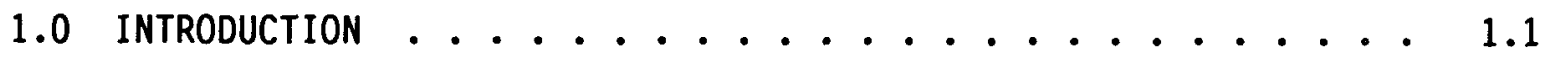

1.1 DEVELOPMENT OF THE GENII SYSTEM $\ldots \ldots \ldots$

1.2 QUALITY ASSURANCE ISSUES . . . . . . . . . . 1.5

2.0 CONCEPTS IN ENVIRONMENTAL DOSIMETRY . . . . . . . . 2.1

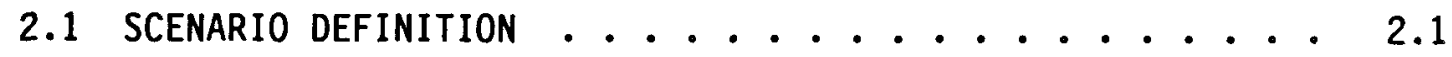

2.1.1 Far-field Scenarios ........... 2.1

2.1.2 Near-Field Scenarios . . . . . . . . 2.2

2.2 EXPOSURE PATHWAYS .................... 2.3

3.0 CODE FUNCTIONAL DESCRIPTIONS . . . . . . . . . 3.1

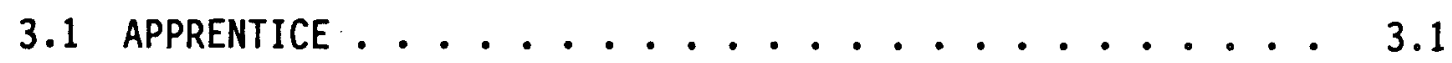

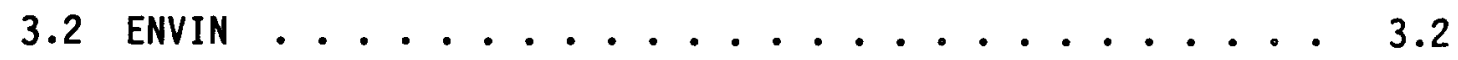

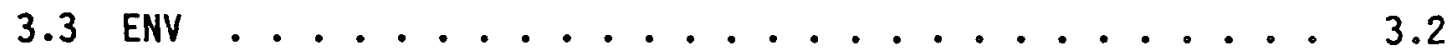

3.4 DOSE . . . . . . . . . . . . . 3.3

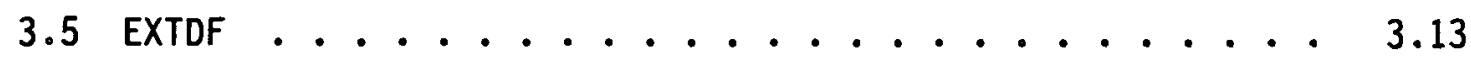

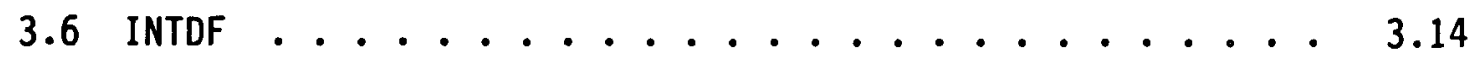

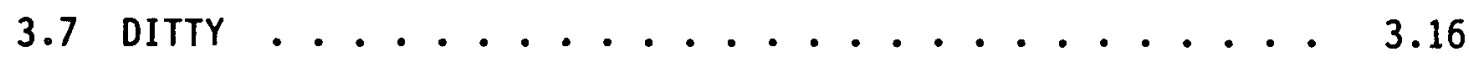

4.0 MATHEMATICAL REPRESENTATIONS ................... 4.1

4.1 INTERNAL DOSIMETRY . . . . . . . . . . 4.1

4.1.1 The General Model .............. 4. 4

4.1.2 The Alkaline Earth Model . . . . . . . 4 4.6

4.1.3 The Iodine Model ............ 4.9

4.1.4 The Tellurium/Iodine Model . . . . . . . 4.9

4.1.5 The Radium/Radon Model .......... 4.13 
4.1.6 The Tritium and Carbon Models ......... 4.13

4.1 .7 Decay Data . . . . . . . . . . . 4.15

4.1 .8 Metabolic Data . . . . . . . . . 4.16

4.1 .9 Dosimetric Data . . . . . . . . . 4.17

4.2 EXTERNAL DOSIMETRY . . . . . . . . . . 4.19

4.2.1 Point Kernel Integration .......... 4.19

4.2.2 Mixed Mass Attenuation Coefficients ....... 4.21

4.2 .3 The Build-Up Factor ............ . 4.21

4.2.4 Conversion of Surface Dose to Effective
Dose Equivalent . . . . . . . . . . 4.23

4.3 ATMOSPHERIC DISPERSION . . . . . . . . . 4.25

4.3.1 Gaussian Plume Models ........... . 4.26

4.3.2 Diffusion Coefficients ......... 4.30

4.3.3 Effective Release Height ......... 4.31

4.3.4 Building-Wake Diffusion Model ........ 4.34

4.3.5 Deposition and Depletion ......... 4.38

4.3.6 Wind Profiles............. 4.... 49

4.3.7 Atmospheric Dispersion Model Limitations .... . 4.40

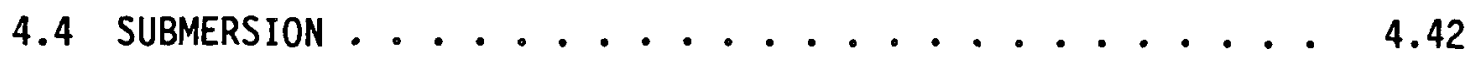

4.4.1 Semi-Infinite Plume Model .......... 4.42

4.4.2 Finite Plume Model ............ 4.43

4.5 SURFACE WATER TRANSPORT MODELS . . . . . . . 4.53

4.6 SOIL CONTAMINATION MODEL . . . . . . . . . 4.56

4.6.1 Deposition Rates .......... 4.57

4.6 .2 Removal Rates ............. 4.58

4.6.3 Biotic Transport ........... 4.59

4.6.4 Manual Redistribution ........... 4.60 
4.7 TERRESTRIAL EXPOSURE PATHWAYS . . . . . . . 4.60

4.7 .1 Inhalation ............... 4.62

4.7.2 Drinking Water Ingestion ........ 4.64

4.7 .3 Aquatic Food Ingestion .......... 4.66

4.7.4 Crop Ingestion - Chronic Exposures ...... 4.66

4.7.5 Animal Product Ingestion - Chronic Exposures ... 4.70

4.7.6 Terrestrial Food Pathways Models - Acute Releases - 4.72

4.7.7 External Exposure . . . . . . . . . 4.76

4.7.8 Population Dose Calculations . . . . . . 4.85

4.7.9 Special Models for $3 \mathrm{H}$ and $14 \mathrm{C} \ldots \ldots . . . . .4 .86$

4.8 LONG-TERM CALCULATIONS . . . . . . . . . 4.90

4.8.1 Incorporation of Long-Term Sources . . . . . 4.90

4.8.2 Long-Term Airborne Releases . . . . . . . 4.91

4.8.3 Long-Term Waterborne Releases . . . . . . 4.92

4.8.4 Long-Term Environmental Concentrations . . . . 4.93

4.8.5 Long-Term Pathway Dose Calculations ....... 4.99

5.0 SOLUTION TECHNIQUES ......................... 5.1

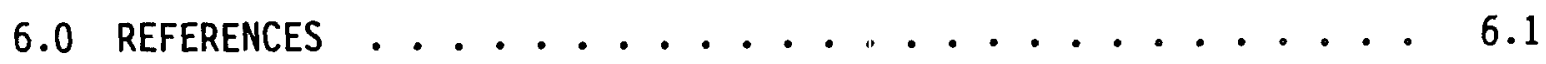

APPENDIX - HEDUP System Design Requirement:s Report ....... A.1 
ABSTRACT

ACKNOWLEDGMENTS

1.0 INTRODUCTION

2.0 CODE STRUCTURE

2.1 USER INTERACTION LEVELS

2.1.1 Level 0 User Interaction - Environmental Dosimetry

2.1.2 Level 1 User Interaction - Environmental Dosimetry

2.1.3 Leve1 1 User Interaction - External Dose Factors

2.1.4 Level 1 User Interaction - Internal Dose Factors

2.1.5 Level 1 User Interaction - DITTY Long-Term Calculations

\subsection{DATA TRANSFER FILE DESCRIPTIONS}

2.2.1 ENVIN/ENV/DOSE InPut File Buffer - GENII. IN

2.2.2 Joint Frequency Input File Buffer - JOINTRE. IN

2.2.3 Chi/Q Input/Report File Buffer - CHIQ.IN

2.2.4 Population Grid Input File Buffer - POP.IN

2.2.5 Food Production Grid Input File Buffer FOODPROD. IN

2.2.6 Parameter Default Value Input File Buffer DEFAULT. IN

2.2.7 ENV Input File Buffer - ENV.IN

2.2.8 INTDF Input File Buffer - INTDF.IN

2.2.9 DITTY Input File Buffer - DITTY.IN

2.2.10 DITTY Water Release Input File Buffer - WATREL. IN 
2.2.11 DITTY Air Release Input File Buffer - AIRREL. IN

2.2.12 EXTDF Input File Buffer - EXTDF.IN

2.2.13 ENV Report File Buffer .. GENII2.0UT

2.2.14 ENVIN Report File Buffer - GENII.OUT

2.2.15 DOSE Report File Buffer - DOSE.OUT

2.2.16 ENV Media Concentration Report File Buffer MEDIA.OUT

2.2.17 DOSE Summary Dose Report File Buffer DOSEQA.OUT

2.2.18 ENV Report File Buffer - ENV.OUT

2.2.19 INTDF Report File Buffer - INTDF.OUT

2.2.20 INTDF CDE Yearly Increment File Buffer CDEINC.OUT

2.2.21 INTDF CDE File Buffer - CDE.OUT

2.2.22 DITTY Report File Buffer - DITTY.OUT

2.2.23 DITTY Summary Dose Report File Buffer DITTYQA.OUT

2.2.24 EXTDF Report File Buffer - EXTDF.OUT

2.3 DATA LIBRARY DESCRIPTIONS

2.3.1 Radionuclide Master Library - RMDLIB.DAT

2.3.2 Metabolic Data Library - METADATA.dAT

2.3.3 Radionuclide List by Atonic Number - RMDBYELE.DAT

2.3.4 Food Transfer Library - FTRANS.DAT

2.3.5 Bioaccumulation Library - BIOAC1.DAT

2.3.6 External Dose Factor Library - GRDF.DAT

2.3.7 Radioactive Decay Data for RMDLIB ENERGY.DAT

2.3.8 DITTY Internal Dose Factor Library DSFCT30.DAT 
2.3.9 Attenuation Coefficients and Build-Up Factor ISOLIB.DAT

2.3.10 Committed Dose Equivalent Yearly Increment Library - DOSINC.DAT

2.3.11 Gamma Energy Library - GAMEN.DAT

2.3.12 Specific Effective Energy Library - SEEn.DAT

2.4 EXTERNAL FILE NAME ASSIGNMENTS - FILENAME.DAT

3.0 USER'S INSTRUCTIONS

3.1 APPRENTICE

3.2 ENVIRONMENTAL DOSIMETRY PACKAGE: ENVIN/ENV/DOSE

3.3 EXTDF

3.3.1 Title

3.3.2 NAMELIST

3.3.3 Shield Material Specification

3.4 INTDF

3.5 DITTY

3.5.1 Case Structure Input

3.5.2 NAMELIST INPUT Parameters

3.5.3 Other Input Parameters

4.0 SYSTEM REQUIREMENTS

5.0 QUALITY ASSURANCE (QA)-RELATED TOPICS

6.0 REFERENCES

APPENDIX A - SAMPLE INPUT AND OUTPUT

APPENDIX B - SELF-GENERATED DIAGNOSTICS 
ABSTRACT

ACKNOWLEDGMENTS

1.0 INTRODUCTION

2.0 CODE STRUCTURE AND LOGIC DIAGRAMS

2.1 SOFTWARE CONFIGURATION CONTROL;, TESTING, AND VERIFICATION

3.0 GLOBAL DICTIONARY AND COMMON BLOCK USAGE

4.0 HAND CALCULATION WORKSHEETS

5.0 LISTINGS

5.1 CODE LISTINGS

APPRENTICE

APPRENTICE

ENVIN

ENV

DOSE

DITTY

INTDF

EXTDF

UNFORMAT

UNSEE

GENERAL

COMMON BLOCKS

5.2 DATA FILE LISTINGS

RMDLIB.DAT

METADATA.DAT 
RMDBYELE.DAT

FTRANS.DAT

BIOAC1.DAT

GRDF.DAT

DOSSUM.DAT

DEFAULT . IN

ENERGY.DAT

DSFCT30.DAT

ISOLIB. DAT

DOSINC.OUT

GAMEN.DAT

SEE. IN

PARAMS.DAT

APPRENTICE.DAT 
FIGURES - VOL. 1

1.1 Current User/Computer Program Interaction in the

GENII Software Package ... . . . . . . . . . . 1.3

2.1 Available Exposure Pathways in the CiENII Package . . . . . 2.4

3.1 Situation Showing Constant Rate of Radionuclide Intake and Resulting One-Year Dose Rate to Tissue or Organ . . . . 3.5

3.2 Situation Showing Committed Dose from One-Year Internal and External Exposure Plus Extended Internal Dose . . . . 3.6

3.3 Situation Showing Cumulative Dose Resulting from Residual Environmental Contamination . . . . . . . 3.8

3.4 Situation Showing Radionuclide Release That Continues for More Than One Year .. . . . . . . . . . 3.9

3.5 Situation Showing Maximum Annual Dose from Continuing Exposure ............... 3.11

3.6 Conceptual Matrix Showing Calculation of Annual, Committed, and Cumulative Doses ............. 3.12

3.7 Calculational Scheme for Long-Term Integrated Population Doses..................... 3.19

4.1 The General Model ................. . . . 4.2

4.2 The Alkaline Earth Model . . . . . . . . . . 4.7

4.3 The Iodine Model ................. 4.10

4.4 The Tellurium/Iodine Model . . . . . . . . . . . 4.12

4.5 Point Source (So) With a Finite Cylindrical Volume ( $\left.S_{V}\right)$ at a Distance $p$ from Point $P$ Through Shields of Thickness $\mathrm{t} 1$ and $\mathrm{t} 2 \ldots$

4.6 Schematic of Soil Compartment Interactions . . . . . . 4.57 
TABLES - VOL. 1

3.1 Available EXTDF Geometries ................ 3.14

4.1 Equations for the General Model Using Parameters

Defined on Page $4.3 \ldots \ldots \ldots . \ldots . . \ldots 4$

4.2 Deposition Fractions and Clearance Half Times $\left(t_{1 / 2}\right)$ for the Lung Model .................. 4.5

4.3 Equations for the Alkaline Earth Model ......... 4.8

4.4 Equations for the Iodine Model ............ 4.11

4.5 Equations for the Tellurium/Iodine Model . . . . . . . 4.14

4.6 Equations for the Radium/Radon Model . . . . . . . . 4.14

4.7 Photon Energy Group Definitions Conversion Factors Used

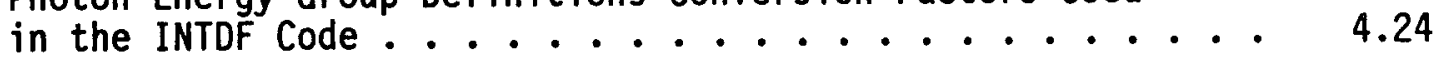

4.8 Constant Values for Diffusion Coefficient Evaluation . . . 4.31

4.9 Photon Energy Group Definitions and Conversion Factors Used in Finite-Plume Air Submersion Calculations ..... 4.44

4.10 Empirical Constants for the Ground-Level Correction Factor, $R_{j} \ldots \ldots \ldots . . . . . . . . . . .44 .45$

4.11 Empirical Constants for Effective Release Height, $H^{*} j$. . . 4.46

4.12 Empirical Constants for Ground-Level Sector Dose Rate Factors .................. 4.48

4.13 Values for "S" Parameters . . . . . . . . . . . 4.48

4.14 Parameter Ahj Values ............. . . 4.49

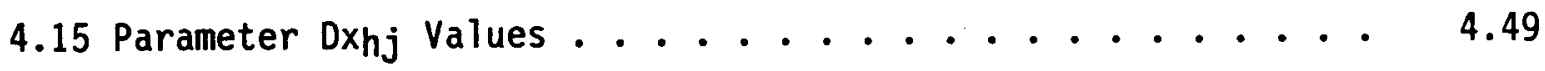

4.16 Parameter $x 1_{h j}$ Values .................... 4.49

4.17 Parameter $x 2 h j$ Values ............. 4.50

4.18 Parameter $x 3 h j$ Values ............. 4.50

4.19 Parameter $X 4 h j$ Values .................. 4.50

4.20 Parameter $x 5_{h j}$ Values ............... 4.51

4.21 Parameter $x 6 h j$ Values ..................... 4.51 
4.22 Parameter $x 7_{h j}$ values ................ 4.51

4.23 Parameter $x 8_{h j}$ values .................. 4.52

4.24 Parameter $x 9 h j$ values . . . . . . . . . . 4.52

4.25 Harvested Yield, Standing Biomass, and Ration of Dry to Wet Vegetation Used in Interception and Harvest

Removal Models ............... 4.71

4.26 Default Consumption Rates of Feed and Water by Farm Animals . 4.72

4.27 Acute Concentration Equations for Leafy Vegetables . . . 4.77

4.28 Acute Concentration Equations for 0ther Vegetables . . . 4.78

4.29 Acute Deposition Integrated Concentration Equations
for Milk and Beef ... 4.79

4.30 Acute Deposition Integrated Concentration Equations

for other Animals................ 4.81

4.31 Suggested Values for Shore-Width Factor . . . . . . 4.83

4.32 Soil Ingestion by Age .................. 4.84

4.33 Fractions of Hydrogen and Carbon in Environmental Media,

Vegetation, and Animal Products.......... 4.88 


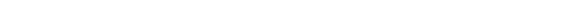




\subsection{INTRODUCTION}

At the direction of the U.S. Department of Energy (DOE), the Hanford Environmental Dosimetry Upgrade Project was undertaken by Pacific Northwest Laboratory (PNL) (a) to incorporate the internal dosimetry models recommended by the International Commission on Radiological Protection (ICRP) in updated versions of the environmental pathway analysis models used at Hanford. The resulting second generation of Hanford environmental dosimetry computer codes is compiled in the Hanford Environmental Dosimetry System (Generation II or GENII). The GENII system was developed by means of tasks designed to provide a state-of-the-art, technically peer-reviewed, documented set of programs for calculating radiation doses from radionuclides released to the environment. The initial task resulted in a system design requirements report, based on input from the community of potential Hanford users, providing general descriptions of the calculations that the final programs must perform. The recommendations of this report formed the basis for the remainder of the tasks, defining the elements that determined the equation formulation and parameter selection tasks. The complete report, Hanford Environmental Dosimetry Upgrade Project (HEDUP) Task 02 - System Design Requirements, is included here as the appendix.

The general requirements of the GENII system to be designed included the capabilities for calculating radiation doses for acute releases, with options for annual dose, committed dose, and accumulated dose; for calculating the same types of doses from chronic releases; for evaluating exposure pathways including direct exposure via water (swimming, boating, and fishing), soil (surface and buried sources), air (semi-infinite cloud and finite cloud geometries), inhalation pathways, and ingestion pathways. The release scenarios to be included were acute releases to air from ground level or elevated sources, or to water; chronic releases to air from ground level or elevated sources, or to water; and initial contamination of soil or surfaces. Source term variations to be accounted for included decay of radionuclides to the start of the exposure scenario, input of total radioactivity or specified

(a) Pacific Northwest Laboratory is operated by Battelle Memorial Institute for the U.S. Department of Energy under Contract DE-AC06-76RLO 1830. 
fractions, and input of measured concentrations in specified environmental media. Interfaces were to be provided for external calculations of atmospheric dispersion, geohydrology, biotic transport, and surface water transport. Target populations were to be identified by distance and direction for individuals, populations, and for intruders into contained sources.

As a result of the multitude of initial requirements on the design of the codes, the codes of the Hanford Environmental Dosimetry System can be used to determine radiation doses to individuals or populations from a wide variety of potential exposure scenarios. The core system may be used to calculate annual doses, dose commitments, or accumulated doses from acute or chronic releases of radioactive materials to air or water. This capability essentially replicates that provided in the past by the computer codes KRONIC (Strenge and Watson 1973), SUBDOSA (Strenge, Watson, and Houston 1975), DACRIN (Houston, Strenge, and Watson 1974; Strenge 1975), FOOD and ARRRG (Napier et a1. 1980), and PABLM (Napier, Kennedy, and Soldat 1980). Annual doses, dose commitments, and accumulated doses can also be calculated from initial contamination of soil or surfaces, thus incorporating capabilities from PABLM and ONSITE/MAXI (Napier et al. 1984; Kennedy et al. 1986; Kennedy et al. 1987). A limited biotic transport capability is included, simulating the results of BIOPORT/MAXI (McKenzie et al. 1986). One of the codes creates factors relating sources of various geometries to dose rate; this is essentially a modified version of the shielding code ISOSHLD (Engel, Greenborg, and Hendrickson 1966; Simmons et al. 1967). An additional capability, that of predicting very-long-term doses from waste management operations to the public over periods of up to 10,000 years, is incorporated into the system essentially unchanged from the previously documented version of DITTY (Dose Integrated over Ten Thousand Years) (Napier, Peloquin, and Strenge 1986).

The Hanford Environmental Dosimetry System (GENII) is composed of seven linked computer codes and their associated data libraries. These codes and their linkages are illustrated in Figure 1.1. The computer programs are of three types: user interfaces (i.e., interactive, menu-driven programs to assist the user with scenario generation and data input), internal and external dose factor generators, and the environmental dosimetry programs. For maximum flexibility, the portion of the code used for analys is of 


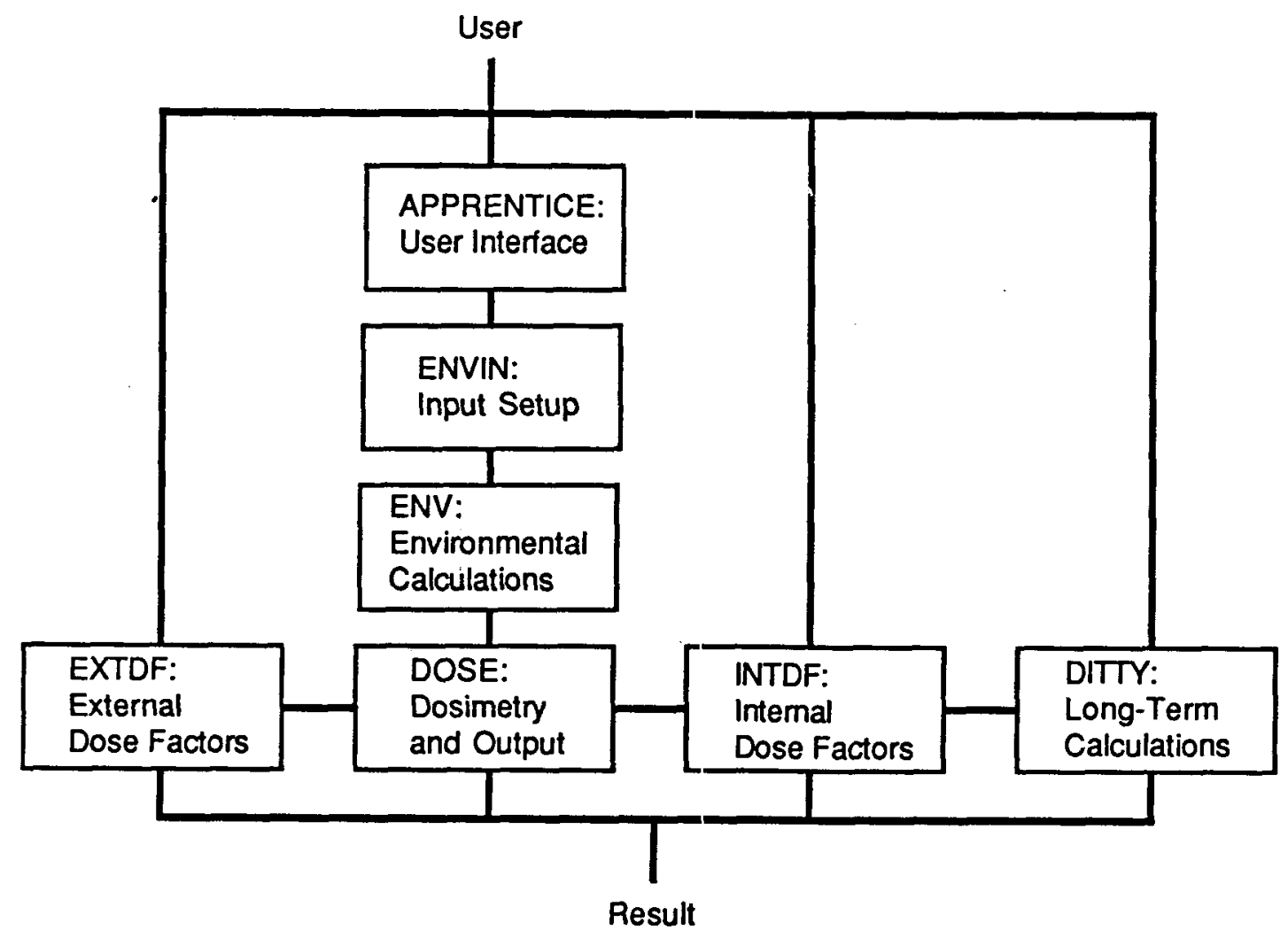

FIGURE 1.1. Current User/Computer Program Interaction in the GENII Software Package

short-term scenarios (as opposed to 10,000-year migration analyses) has been divided into three interrelated but separate programs that handle input organization and checking, environmental exposure, and dose calculations, respectively. The intent of each of these codes is described in Section 3.0 of this document.

GENII is described in three volumes of documentation. This volume describes the theoretical considerations of the system, including the conceptual diagrams, mathematical representations of the solutions, and descrip-. tions of solution techniques, where appropriate. The second volume is a Users' Manual, providing code structure, users' instructions, required system configurations, and topics related to quality assurance (QA). The third volume is a Code Maintenance Manual for the user who requires knowledge of code details, including code logic diagrans, global dictionary, worksheets, 
example hand calculations, and listings of the code and its associated data libraries.

\subsection{DEVELOPMENT OF THE GENII SYSTEM}

A comprehensive set of codes (i.e., environmental pathway models and associated computer programs) has been developed and documented by PNL for estimating potential radiation doses to humans as a result of radionuclides in the environment. The codes address both routine and accidental releases of radionuclides to air or water in addition to other situations $(\mathrm{e} . \mathrm{g}$. , residual contamination from spills or decommissioning operations). Internal radiation dose calculations are performed using the methods recommended by the ICRP as described in ICRP 26 and 30 (ICRP 1977; ICRP 1979-1982). The codes are based on existing Hanford models and codes, using updated formulations and transfer coefficients. Existing codes incorporated include DACRIN (Houston, Strenge, and Watson 1974; Strenge 1975), PABLM (Napier, Kennedy, and Soldat 1980), MAXI (Napier et al. 1984), DITTY (Napier, Peloquin, and Strenge 1986), SUBDOSA (Strenge, Watson, and Houston 1975), KRONIC (Strenge and Watson 1973), and ISOSHLD (Engel, Greenborg, and Hendrickson 1966). A user-friendly, interactive, driver program was developed to consolidate all code input and output requirements. The resultant codes are small enough to operate on a personal computer. All codes and data bases developed in the PNL project have been thoroughly peer-reviewed, tested, and documented.

The goal of this project was to develop a new generation of multipurpose mathematical models and computer programs. The resultant codes will be used for retrospective calculations of potential radiation doses resulting from routine Hanford emissions by groups within PNL, as well as by other Hanford contractors. The codes will also be used for prospective dose calculations for purposes such as siting facilities, environmental impact statements (EISs), and safety analysis reports (SARs). It was important that each user group provide input to the design of the proposed codes.

A task was initiated to define the system design requirements of the proposed codes. A committee of representatives from potential user groups was formed under the guidelines of the Hanford Dose Overview Program. 
Recommendations of this group were incorporated with those of prospective PNL users to help define the needed capabilities.

Based on the recommendations developed, the relationships between radionuclide release, flux, concentration, and uptake were formulated. The pathways included are internal exposure through inhalation, crop ingestion, animal product ingestion, water consumption, and aquatic food product ingestion; and external exposure through air submersion, water immersion (swimming), and contaminated ground surfaces and objects. The equations are used as the basis for subsequent computerized implementation.

Solving the equations depends on the values of the transfer parameters used. Existing libraries of radionuclide-specific factors existed but had not been reviewed/updated for nearly 10 years. A literature review was performed of the most recent pertinent information with emphasis on Hanfordspecific data. Parameters investigated included soil-to-plant concentration ratios (CRs), plant-to-animal product CRs, aquatic bioaccumulation factors, resuspension rates and resuspension factors (including mass loading rates), and selected specific-activity models (e.g., hydrogen-3 and carbon-14).

The most flexible structure for a new generation of environmental dosimetry models is similar to that shown as Figure 1.1. The major structural components are an environmental transport and human exposure module (incorporating most of the existing mode1s) coupled to an internal dosimetry module. For convenience and ease of use, these are controlled by an interactive "driver" program. Most data inputs are through calls to data files (not shown in Figure 1.1).

\subsection{QUALITY ASSURANCE ISSUES}

The GENII package of codes was developed under a stringent QA plan based on the American National Standards Institute (ANSI) standard NQA-1 (ASME 1986) as implemented in the PNL Quality Assurance Manual PNL-MA-70. (a) A11 steps of the code development have been documented and tested. Extensive

(a) Procedures for Quality Assurance Program, PNL-MA-70, is a controlled document used internally at PNL. Information regarding the manual may be obtained from the Pacific Northwest Laboratory, Richland, Washington. 
hand calculations have been performed and are available for review. An external peer review of the internal dosimetry portions of the code was held in Richland, Washington, the week of September 14, 1987. A second external review of the entire package was held in Richland the week of February 1, 1988. Copies of the reports issued by these reviewers are available. Recommendations of these review committees were then incorporated into the final product. 


\subsection{CONCEPTS IN ENVIRONMENTAL DOSIMETRY}

Some basic interrelated concepts were used to develop the GENII code system, some explicitly labeled and some implicitly used. These concepts of "Exposure Scenario Definition" and "Exposure Pathiways" are described in this section. Understanding the approaches taken to allow flexible scenario definition will aid the user in applying the codes. The general concepts of "scenarios" are described first, followed by description of the environmental pathways included in GENII.

\subsection{SCENARIO DEFINITION}

A "scenario" is a conceptual model that calculates patterns of human activity corresponding to actions, events, and processes that result in radiation exposure to individuals or groups of people. The GENII code package can evaluate doses resulting from two general scenario types: farfield and near-field. A far-field scenario is defined as one that determines the impact of a particular release of radioactive material into a wide environment, such as doses from releases from a stack to individuals or populations downwind. In a near-field scenario, the focus is on the doses an individual could receive at a particular location as a result of initial contamination or external sources, i.e., buried waste or contaminated soil. In a near-field scenario, contamination levels in specific environmental media may be known. A far-field scenario focuses outward from a source, and a near-field scenario focuses in toward a receptor. Of course, the two types are not mutually exclusive. Some doses to individuals from remote sources can be calculated as either far- or near-field with the same result. Specific examples of common types of far-field and near-field scenarios are given below.

\subsubsection{Far-Field Scenarios}

A number of commonly encountered scenarios may be classed as far-field. The following are types of releases of radioactive materials into the wider environment:

- chronic atmospheric releases - prospective or retrospective doses to individuals or populations at specified distances and directions 
from the radioactive source, via submersion, inhalation, deposition groundshine, and food pathways. This class of scenario is commonly used for demonstrating compliance to regulations that involve emission sources.

- acute atmospheric releases - prospective or retrospective doses to individuals or populations at specified distances and directions from an acute radioactive release, via submersion, inhalation, deposition groundshine, and food pathways. This class of scenario is often used in SARs or EISs.

- chronic surface water releases - prospective or retrospective doses to individuals and populations at specified distances downstream of a release point via swimming, boating, shoreline, drinking water, aquatic foods, irrigated terrestrial foods and animal products, soil contaminated via irrigation, and other pathways associated with liquid releases. This class of scenario is also often encountered during the demonstration of compliance with regulations.

- acute surface water releases - prospective or retrospective doses to individuals and populations at specified distances downstream of a release point via swimming, boating, shoreline, drinking water, aquatic foods, irrigated terrestrial foods and animal products, soil contaminated via irrigation, and other pathways associated with acute liquid releases. This class of scenario is also associated with SARs and EISs.

\subsubsection{Near-Field Scenarios}

Most other types of scenarios may be considered to be near-field. Examples are:

- initial surface contamination - individual doses resulting from contact with contaminated soil or surfaces via direct contact, resuspension, or crop uptake. Calculations of this nature can be used to analyze the impact of spills or remedial actions.

- initial subsurface contamination - individual doses resulting from contact with contaminated soil or surfaces via direct contact, 
resuspension, or crop uptake. The surface soil may be contaminated via manual redistribution of the material or through biotic transport. Calculations of this nature can be used to analyze the impacts of waste management options. Often a time delay may be included to account for radioactive decay of the source.

- ground water contamination - prospective or retrospective doses to individuals and populations from a given water concentration via drinking water, irrigated terrestrial foods and animal products, soil contaminated via irrigation, and other pathways associated with liquid releases. This class of scenario is also often used in demonstrating compliance with regulations.

- cumulative effects - prospective calculations combining initial soil contamination with additional contributions from an external atmospheric or liquid source. This type of calculation is representative of how near-field and far-field calculations can conceptually be combined.

As seen in the above examples, the near-field and far-field scenarios are not necessarily mutually exclusive, and in some instances either approach may be used in defining them. An interactive user interface is provided with the GENII package to aid the user in scenario definition.

\subsection{EXPOSURE PATHWAYS}

Potential routes through which people may be exposed to radionuclides or radiation are called "exposure pathways." Examples of general pathways are external exposure, inhalation, and ingestion. The pathways are defined depending on the ways people could be exposed for a given circumstance. In this way, an appropriate collection of defined pathways can also be considered as the definition of an "exposure scenario." Because a conceptualization of the types of exposure must be made before the pathways can be fully defined, "scenarios" are usually defined before the parameters are selected for the "pathways."

The various exposure pathways available to users of the GENII package are illustrated in Figure 2.1. This logic diagram shows how known or assumed 


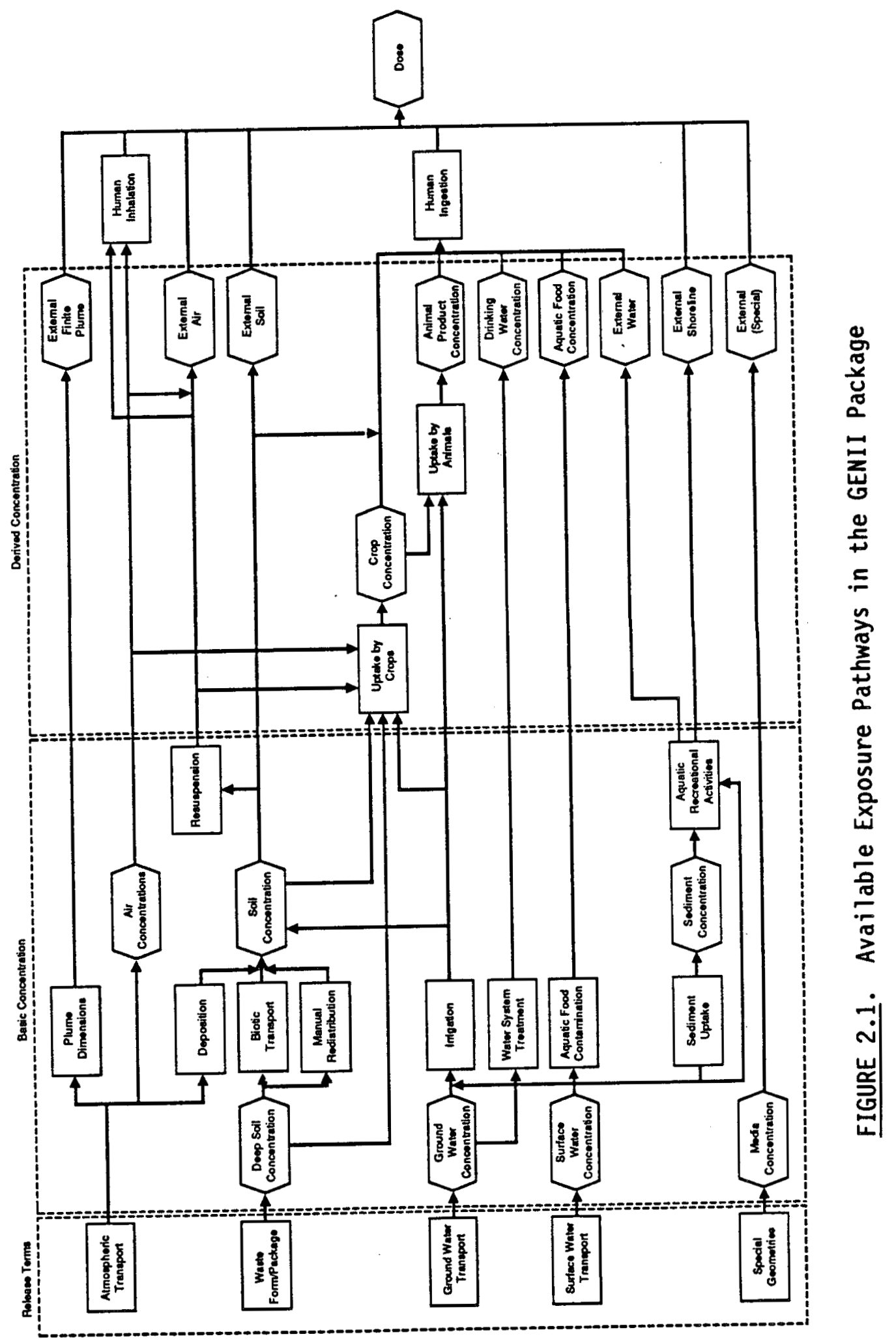


values may be propagated through defined steps to estimate radiation doses. In the GENII formulation, data regarding the radioactive release or radioactive contamination levels may be input to the calculations at different points in the calculation. These are represented on the diagram as hexagonal boxes.

In most calculations, the known quantity is generally the release quantity, designated the "Release Terms" in Figure 2.1. These terms may take the form of activity, or activity per unit time, released into the transport media of air, surface water, ground water, or soil. They are converted via appropriate models to concentrations in the corresponding media (if the basic media concentration is known, it may be input and the transport step omitted). The processes represented by square boxes in Figure 2.1 are simulated, resulting in the "Derived Concentrations." Again, if the Derived Concentrations are known, the earlier processes may be overridden. Finally, the human exposure parameters are factored in, and the dose is estimated.

To optimize flexibility, the capability to input precalculated values of atmospheric dispersion, ground water concentration, and surface water concentration has been included in the GEIII package. In this way, if the simple methods incorporated in the GENII package are insufficient for a particular calculation, more sophisticated results may be achieved with minimal effort. 



\subsection{CODE FUNCTIONAL DESCRIPTIONS}

The GENII system is composed of seven interrelated computer codes and their associated data libraries, as illustrated in Figure 1.1. The connection between the codes is through data transfer files. The output of one code is stored in a file that can be real by the next code in the system. Depending on the desired usage, the experienced user may also stop the process and edit the intermediate data transfer files to obtain specific information on the processes occurring or on intermediate results. Input and output details for each of the codes are provided in the Users' Manual, Volume 2 of the GENII documentation.

Section 3.0 describes the general functions and capabilities of each of the separate codes.

\subsection{APPRENTICE}

Detailed input to the GENII system consists of a multi-page data file containing the parameterized scenario description, radionuclide inventory data, and control parameters desired for a particular calculation. The APPRENTICE code is a not-quite-expert system that helps the user, through a series of interactive menus and questions, to prepare a text input file for the environmental dosimetry programs. In addition, APPRENTICE prepares a batch processing file to manage the file handling needed to control the operations of subsequent codes and prepare an output report.

Novice users will appreciate the pop-up help screens that are available in all sections of the program, the extensive logic that requests only pertinent input, default values available for all parameters, reasonable parameter value ranges, error checking for scenario incompatibilities, and checking for validity of file names. Experienced users will find that APPRENTICE has been constructed so that tutorial information does not get in the way of efficient scenario preparation. Pull-down menus allow the user substantial flexibility to modify a scenario under construction. The user can create multiple input files to execute under the control of a single batch-processing file that is generated by APPRENTICE in a relatively unobtrusive manner. File management is available within APPRENTICE; the 
user may view, copy, and rename files, as well as make subdirectories and change default paths.

\subsection{ENVIN}

The ENVIN module of the GENII package controls the reading of the input files optionally prepared by APPRENTICE and organizes the input for optimal use in the following environmental transport and exposure module, ENV. The ENVIN code interprets the basic input, reads the basic GENII data libraries and other optional input files, and organizes the input into sequential segments based on radionuclide decay chains.

Input to ENVIN occurs by means of a standardized file containing scenario, control, and inventory parameters. Radionuclide inventories can be put in as functions of release to air or water, concentration in basic environmental media (air, soil, or water), or concentration in foods. If certain atmospheric dispersion options have been selected, this module can generate tables of atmospheric dispersion parameters $(X / Q)$ that will be used in later calculations. If the finite plume air submersion option is requested in addition to the atmospheric dispersion calculations, preliminary energy-dependent finite plume dose factors are also prepared. ENVIN prepares the data transfer files that are used as input by the ENV module. It also puts out the first portion of the calculation documentation - the run input parameters report.

\subsection{ENV}

The ENV module is the portion of the GENII package that calculates the environmental transfer, uptake, and human exposure to radionuclides that result from the chosen scenario for the defined source term. The code reads the input files from ENVIN, and then for each radionuclide chain sequentially performs the precalculations to establish the conditions at the start of the exposure scenario. Environmental concentrations of radionuclides are established at the beginning of the scenario by assuming decay of pre-existing sources, considering biotic transport of existing subsurface contamination, and defining soil contamination from continuing atmospheric or irrigation depositions. Then, for each year of postulated exposure, the code estimates 
air, surface soil, deep soil, and ground and surface water concentrations of each radionuclide in the chain. Human exposures and intakes of each radionuclide are calculated for the pathways of external exposure from finite or infinite atmospheric plumes; inhalation; external exposure from contaminated soil, sediments, and water; external exposure from special geometries; and internal exposures from consumption of terrestrial foods, aquatic foods, drinking water, animal products, and inaclvertent intake of soil.

The intermediate information on annual media concentrations and intake rates are written to data transfer files. While these may be accessed directly, they are usually used as the input to the DOSE module of GENII.

\subsection{DOSE}

The DOSE module of the GENII package reads the annual intake and exposure rates defined by the ENV module and converts them to radiation dose. The calculation of external dose is done with precalculated factors from the EXTDF module, and the calculation of internal dose is done with precalculated factors from the INTDF module.

Radiation dose is proportional to the quantity of energy deposited per unit mass of irradiated tissue. The total "absorbed dose" is reported in units of "rad" (100 ergs/g) or, as in the International System of Units, commonly known as SI units, "gray," abbreviated "Gy" $(1 \mathrm{~J} / \mathrm{kg})$. This is normalized to relative biological effect, depending on the type of radiation, in "dose equivalent" units of "rem" or "sievert" (Sv) in SI units. The length of time and mode of exposure designates the type of dose calculated.

ICRP 26 states that special considerations must be made "when the exposure is extended in time." The report defines "dose-equivalent commitment" to be the time integral of the dose rate in a given organ or tissue. The report then further defines the "committed dose equivalent" to be the dose that results " from a single intake of radioactive material into the body. This quantity, which may be considered to be a special case of dose-equivalent commitment, is the dose equivalent that will be accumulated over 50 years ... following the intake" (i.e., a 50-year dose commitment). For convenience, a dose equivalent is usually simply called a "dose" (ICRP 1977, p. 6). 
The types of doses calculated in the DOSE module include:

1. One-Year Dose - the population or individual dose that results from one year of exposure (external plus internal). This is the dose currently used for comparison with occupational exposure standards and the one originally used for comparison with public standards.

The one-year dose is now used at Hanford as a measure of potential short-term impact from accidental releases during waste management operations. This situation is illustrated in Figure 3.1, where "Intake" shows a constant rate of radionuclide intake (through inhalation and/or ingestion) and "Dose Rate" shows the resulting dose rate to a tissue or organ. The integral of the dose rate over one year is the one-year dose (i.e., the area under the dose-rate curve).

2. Committed Dose - the population or individual dose that results from one year of external and internal exposure plus the extended internal dose that results from the one-year intake (ingestion plus inhalation). Normally, a 50- or 70-year dose-commitment period is used (i.e., the one-year intake period plus 49 or 69 years). This dose is the one currently being used by most of those who calculate public doses.

The committed dose is used as a measure of the potential, longerterm impact of accidents and routine releases. This situation is illustrated in Figure 3.2, where "Intake" is the same as in Figure 3.1 and shows a constant rate of radionuclide intake over a one-year period. "Dose Rate" in Figure 3.2 shows the resulting dose rate, and the initial portion of the curve is identical to that of Figure 3.1. However, the residual radioactive material left in the body continues to produce a dose after the initial one-year period ends. The integral of the dose rate over 50 or 70 years gives the committed dose that results from one year of intake. 
$\omega$

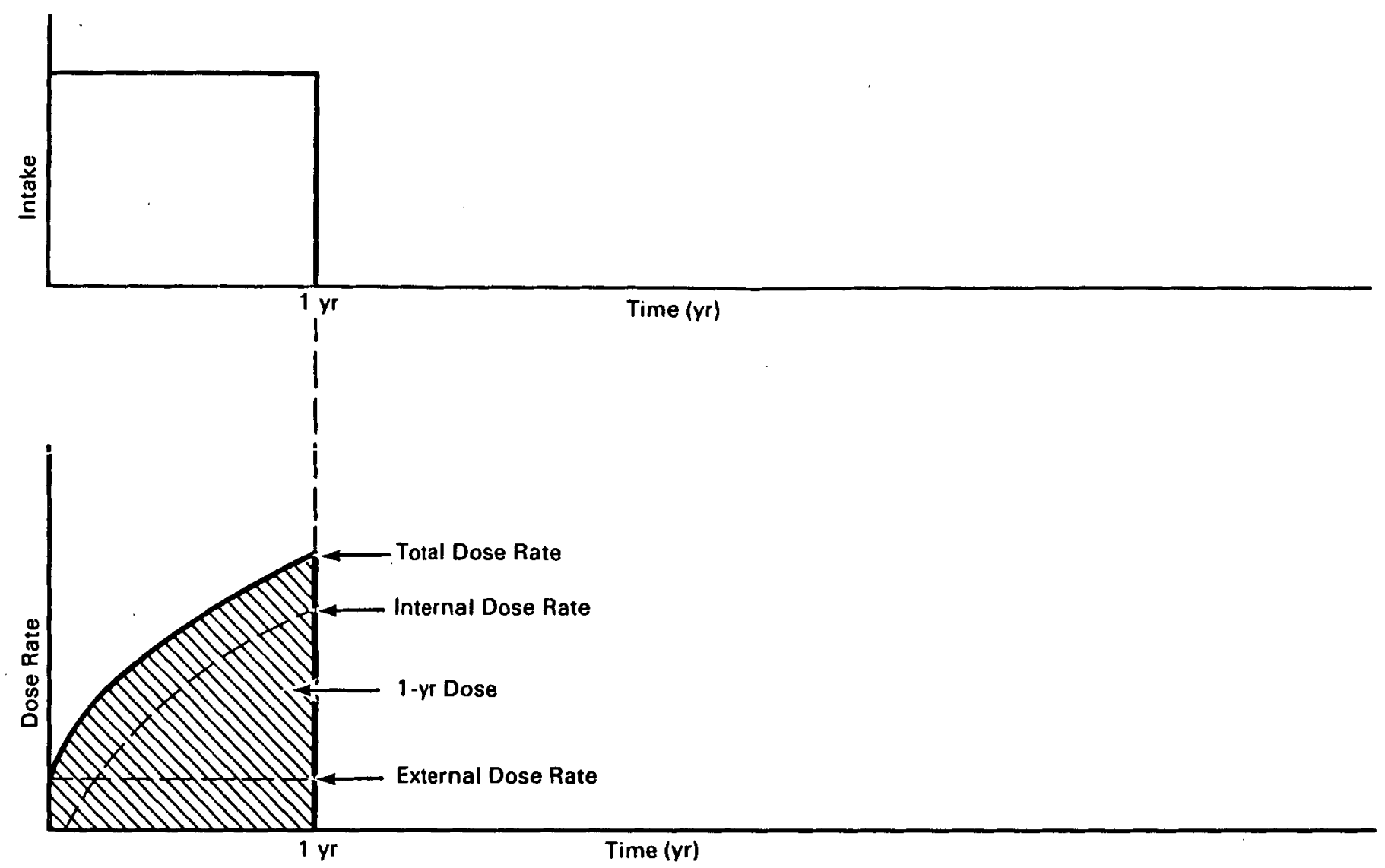

FIGURE 3.1. Situation Showing Constant Rate of Radionuclide Intake and Resulting One-Year Dose Rate to Tissue or Organ 
or

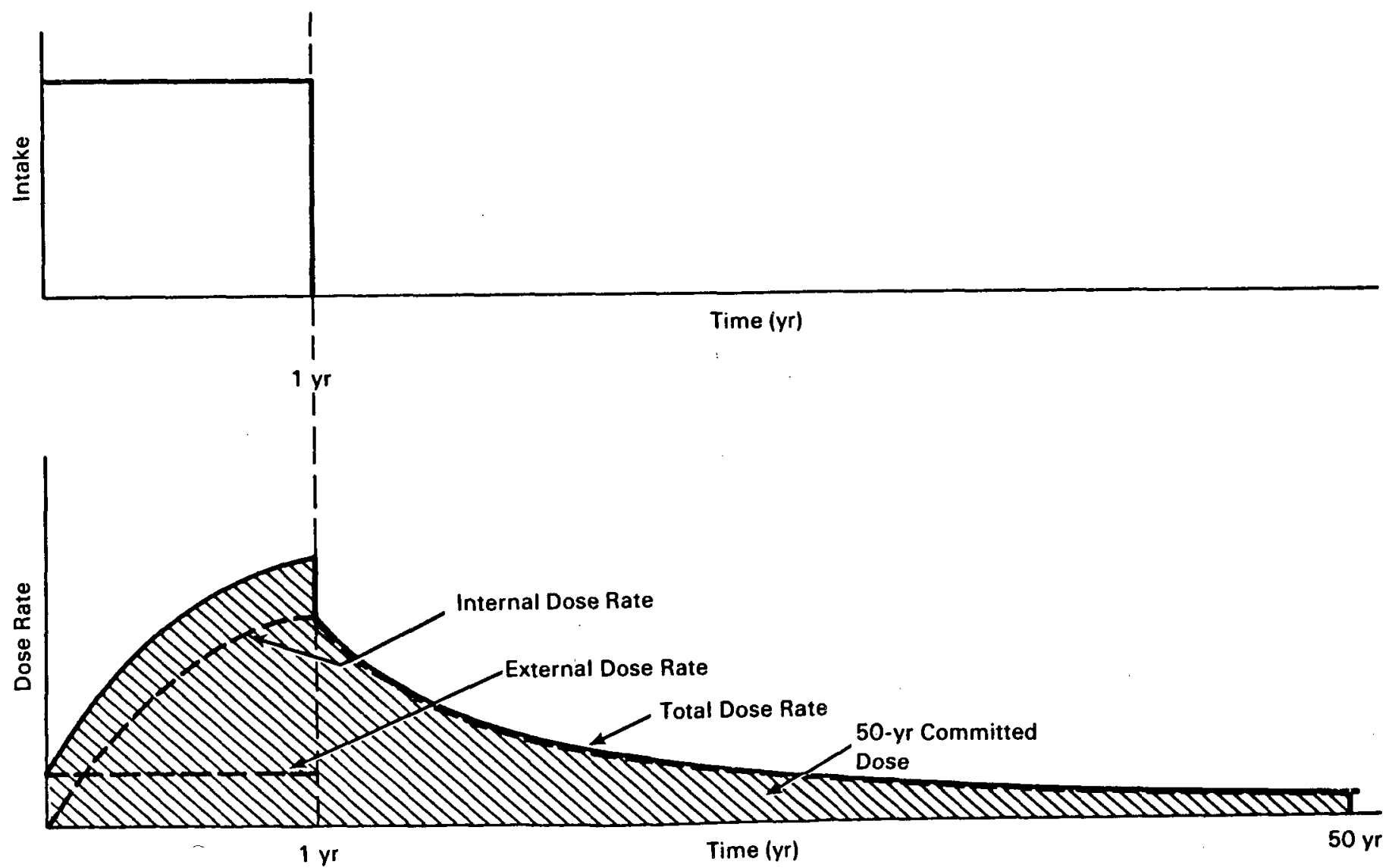

FIGURE 3.2. Situation Showing Committed Dose from One-Year Internal and External Exposure Plus Extended Internal Dose 
3. Cumulative Dose - the population or individual lifetime dose (50- or 70-year) that results from external exposure plus intake via ingestion and inhalation. This includes the dose from radionuclides that accumulate (and physically decay) in the environment during the exposure period. The curnulative dose is used to assess the total potential impacts from operations that have chronic releases over a period of several years or from significant deposition resulting from accidents.

Some parameters can be varied in calculating cumulative dose. One example used in the Hanford annual surveillance reports between CY 1982 and CY 1987 (Jaquish 1987) is a one-year release of radionuclides with resulting residual environmental contamination. The resulting rate of radionuclide intake is illustrated in Figure 3.3, "Intake." The residual environmentally deposited radionuclides decrease by physical decay. The resulting dose rate is illustrated in Figure 3.3, "Dose Rate."

A more complicated example, shown in Figure 3.4, is of a release that continues for more than one year. Scenarios of this sort are routinely used in prospective estimates of the total potential impact of a facility (e.g., environmental impact statements or EISs).

4. Maximum Annual Dose - the largest annual dose that could occur during a specific period (e.g., 50 or 70 years). This calculation accounts for each year's external exposure plus the internal dose from radionuclides taken in during the year of interest and all previous years. The maximum annual dose is identified by including all organ doses. This type of dose estimate corresponds most closely to the existing annual dose limits for occupational and public exposure.

The maximum annual dose is calculated for scenarios that consider human intrusion or long-term casual exposure to disposed wastes, for EISs, and for the allowable residual contamination level (ARCL) evaluations used for decommissioning operations. The maximum 


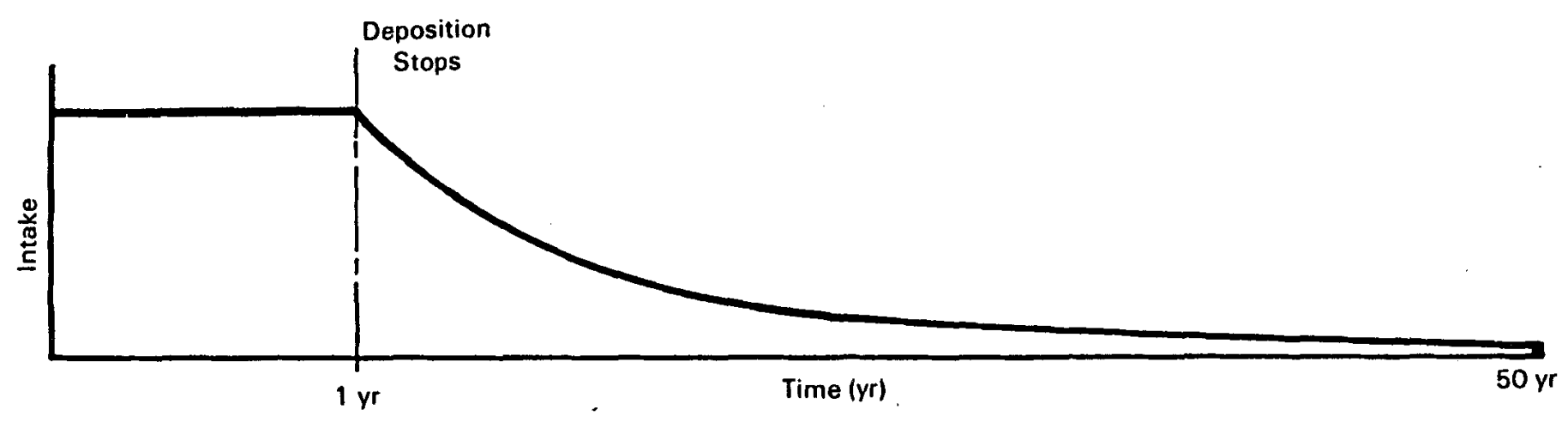

$\infty$

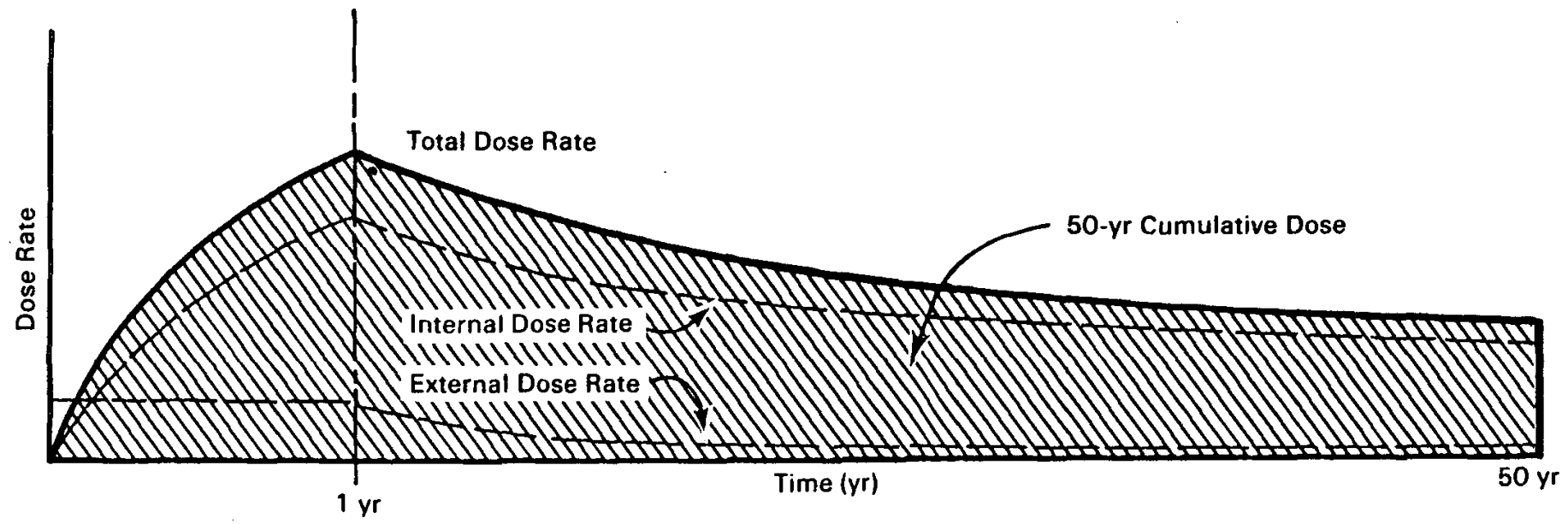

FIGURE 3.3. Situation Showing Cumulative Dose Resulting from Residual Environmental Contamination 

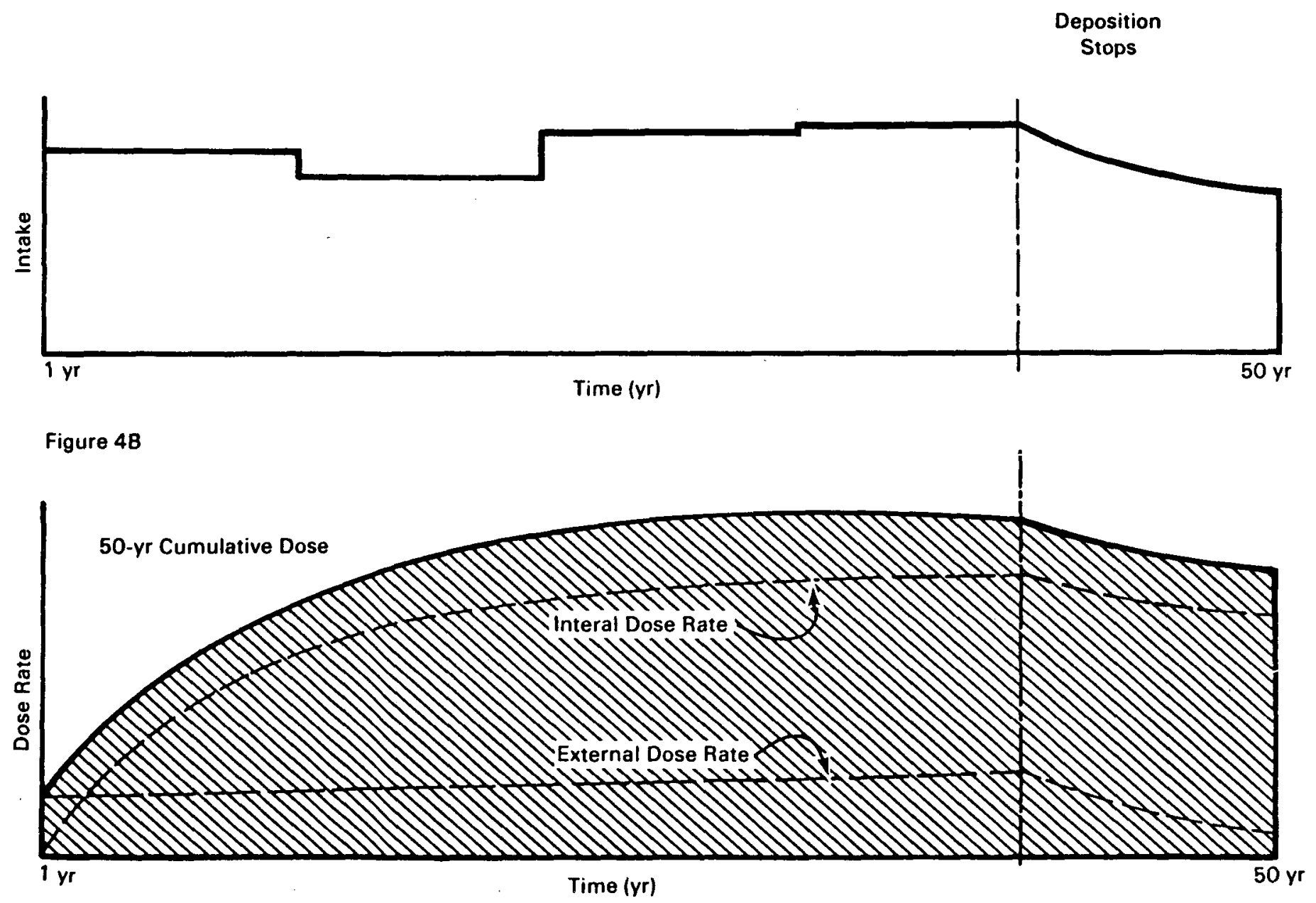

FIGURE 3.4. Situation Showing Radionuclide Release That Continues for More Than One Year 
annual dose is calculated by assuming an initial concentration of radionuclides in soil, concrete, piping, and other materials. These concentrations are reduced over time by physical decay. The annual dose rate to the exposed individual from internally deposited radionuclides increases until the body burden reaches equilibrium with the environmental levels of the physically decaying radionuclides. The year that equilibrium occurs usually corresponds to the maximum dose rate. The maximum-dose-rate year may not occur until many years after the initial exposure. This situation is illustrated in Figure 3.5.

Because annual dose rates are calculated, the dose conversion factors from ICRP 30 are not usable; they are only for 50-year committed doses following an intake. The 50-year dose conversion factors cannot simply be divided by 50 to produce an "annual dose." The ICRP 30 factors are integrals similar to those illustrated in Figure 3.2, depending on the effective half time of the radionuclides in the body (ICRP 1979-1982).

The computer code for calculating the internal dose factors, INTDF, does not itself provide these type of doses. Instead, it is used to calculate the incremental organ doses for each year following an initial intake. The output is the incremental dose to each organ, which is then used in DOSE to assemble annual doses, dose commitments, and accumulated doses. The method by which these doses are assembled is illustrated in Figure 3.6. This technique minimizes the number of calculations needed from the internal dosimetry module.

Effective dose equivalent (EDE) calculated in DOSE is calculated in the manner presented in ICRP 26 (1977). That is

$$
E D E=\sum_{t} w_{t} H_{t}
$$



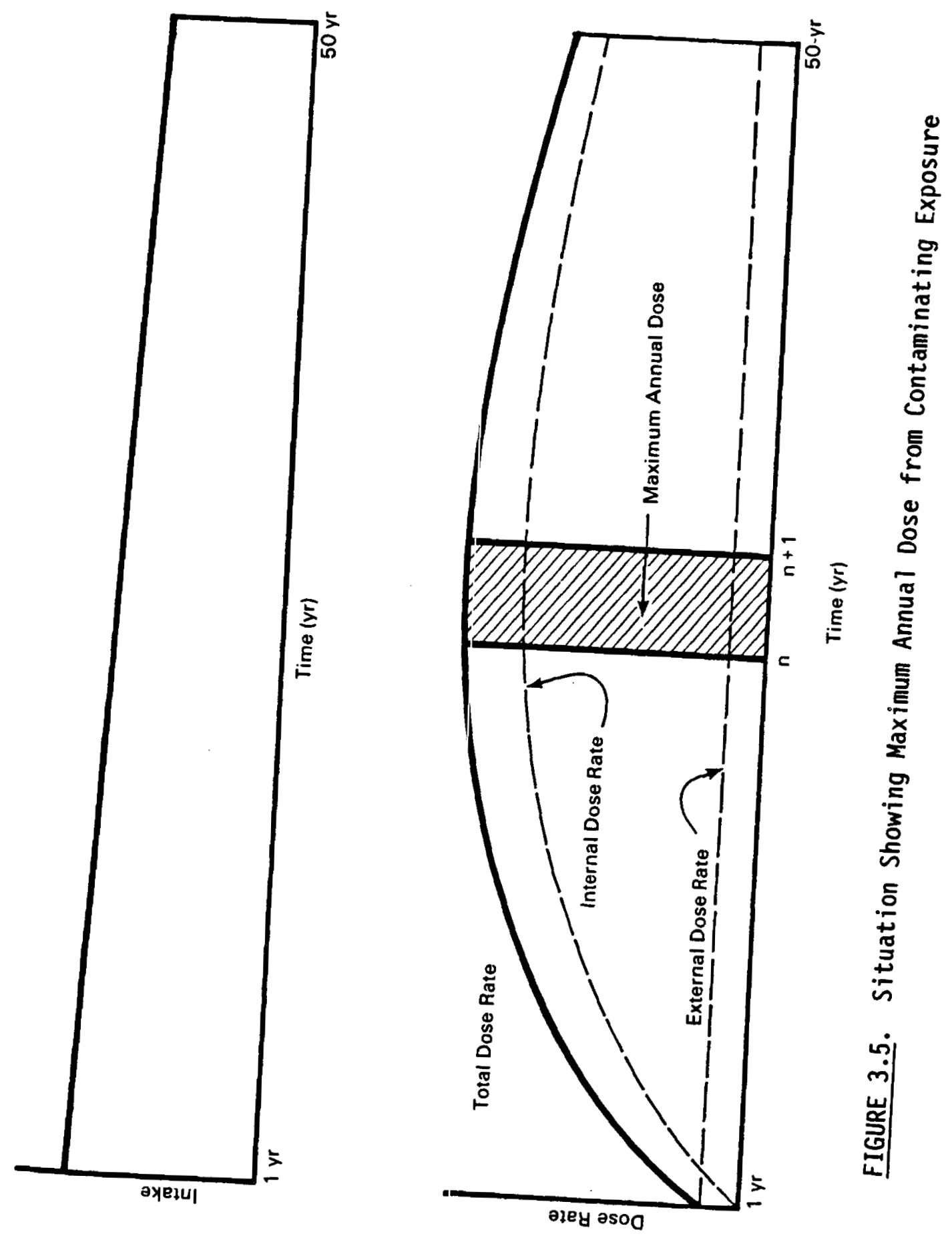


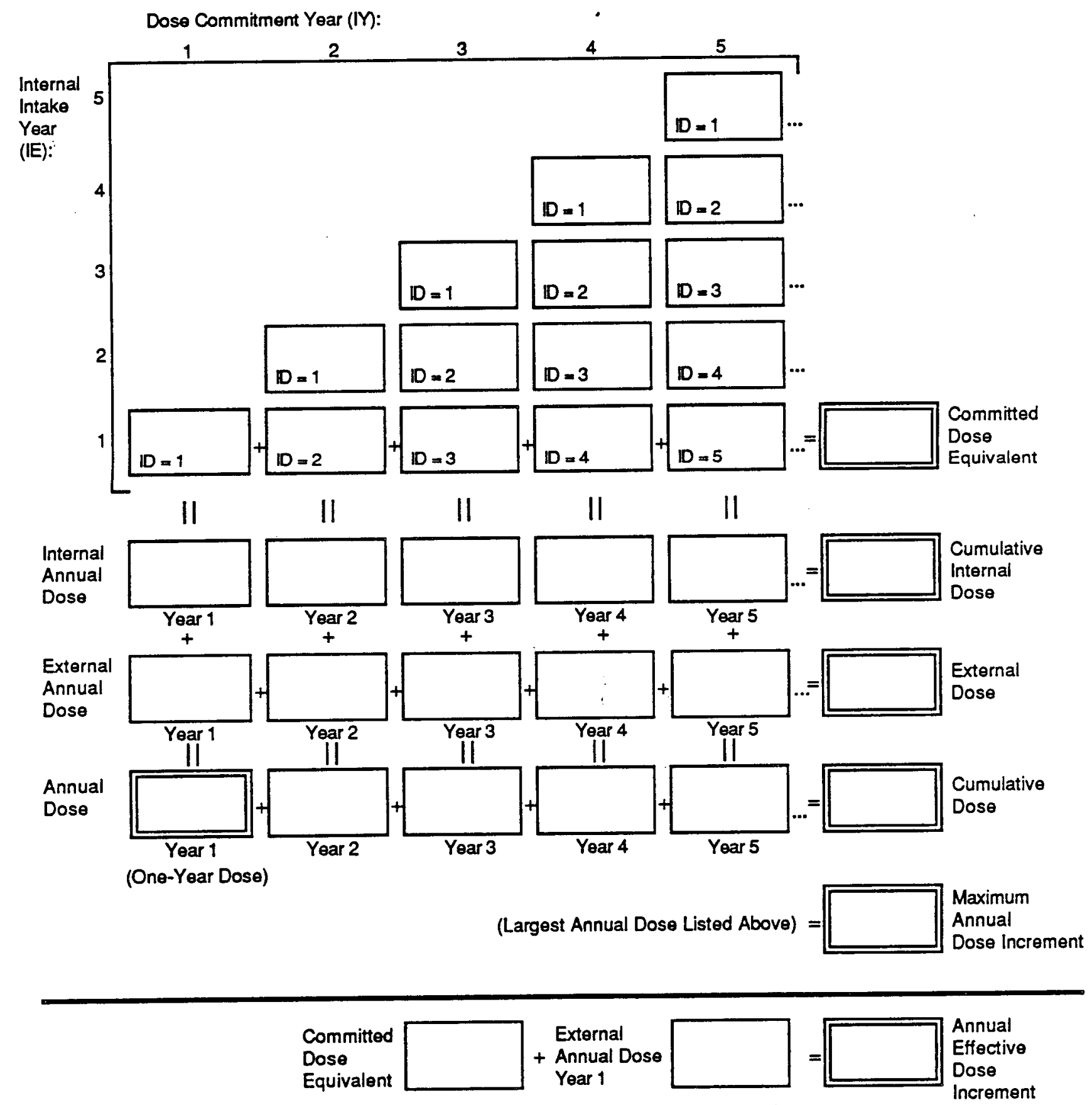

FIGURE 3.6. Conceptual Matrix Showing Calculation of Annual, Committed, and Cumulative Doses 
The weighting factors ( $w t)$ used are also those of the ICRP, given in ICRP 26 (1977) as:

$\begin{array}{ll}\text { Gonads } & 0.25 \\ \text { Breast } & 0.15 \\ \text { Red Bone Marrow } & 0.12 \\ \text { Lung } & 0.12 \\ \text { Thyroid } & 0.03 \\ \text { Bone Surfaces } & 0.03 \\ \text { Remainder } & 0.30\end{array}$

where the "remainder" is applied to the five other organs having the highest dose, with a weighting of 0.06 for each.

DOSE also prepares the remainder of the normal output report of doses, optional doses by pathway and by radionuclide. This output is combined with that from the ENVIN routine to comprise the total routine reports.

\subsection{EXTDF}

The EXTDF module is incorporated into the GENII package to allow easy and uniform calculation of external dose-rate factors for submersion in an infinite cloud of radioactive materials, immersion in contaminated water, and direct exposure to plane or slab sources of contamination. The EXTDF capability also allows for the creation of special dose-rate factors for sources of other geometries in the GENII system. The EXTDF code is a modification and enhancement of the well-known shielding code ISOSHLD (Engel, Greenborg, and Hendrickson 1966). The portion of ISOSHLD that can be used to calculate source strength from reactor fission fuel (the RIBD routines) has been eliminated. The data libraries of radionuclide decay information and gamma and beta yields have been updated from the DRALIST compilation of Kocher (1981b).

The ISOSHLD code uses a point kernel integration technique; i.e., the dose at the exposure point is the contribution from a large number of individual point sources. A numerical integration is carried out over the source volume to obtain the total dose. For a limited number of geometries, an analytical solution is used. The geometries available in the EXTDF are the standard 14 available in ISOSHLD, as listed in Table 3.1. 
TABLE 3.1. Available EXTDF Geometries

\begin{tabular}{|c|c|}
\hline Source Configuration & Shield Configuration \\
\hline Point & Slab \\
\hline Line & Slab \\
\hline Spherical & Spherical \\
\hline Spherical & Slab \\
\hline Truncated Cone & Slab \\
\hline Infinite $\mathrm{Pl}$ ane & Slab \\
\hline Infinite Slab & Slab \\
\hline Disc & Slab \\
\hline Cylindrical (side) & Cylindrical \\
\hline Cylindrical (side) & Slab \\
\hline Cylindrical (side) & Cylindrical and Slab \\
\hline Cylindrical (end) & Slab \\
\hline Rectangular & Slab \\
\hline Annular Cylinder & Cylindrical and Slab \\
\hline
\end{tabular}

EXTDF is 1 imited to calculating normalized dose rate factors. It is not available for routine shielding calculations because it automatically reads and computes factors for unit inventories of all radionuclides in the GENII master nuclide library.

\subsection{INTDF}

The INTDF code is used to make estimates of the dose equivalents in a number of target organs from the activity in a given source organ. For each type of radiation $i$, the dose equivalent $H_{i}$ in target organ $T$ from radionuclide $j$ in source organ $S$ is the product of two factors:

- the total number of nuclear transformations of radionuclide $j$ in organ $S$ over the period of interest following intake

- the energy absorbed per gram in target organ $T$, suitably modified for the quality factor, from radiation of type $i$ per transformation of radionuclide $j$ in source organ $S$. In other words, for each radiation of type $i$ from radionuclide $j$. 


$$
H(T+S)_{i}=U_{S} \cdot 1.6 \cdot 10^{-13} \mathrm{SEE}(T+S)_{i} \cdot 10^{3}
$$

where $\quad U_{S}=$ the number of transformations of radionuclide, $j$, in source organ, $S$, over the period of integration following intake of the radionuclide

$1.6 \cdot 10-13$ = the number of joules in $1 \mathrm{MeV}$

$\operatorname{SEE}(T+S)_{i}$ (in MeV $g^{-1}$ per transformation) $=$ the specific effective energy for radiation type $i$, suitably modified by quality factor, absorbed in $T$ from each transformation in $S$ $10^{3}=$ the conversion factor from $\mathrm{g}^{-1}$ to $\mathrm{kg}^{-1}$.

In general, for the intake of any mixture of radionuclides, i.e. parent with daughters, the dose in target organ $T$ from radioactivity in several different sources $S$ is given by

$$
H=1.6 \cdot 10^{-10} \sum_{S} \sum_{j}\left[U_{S} \sum_{i} \operatorname{SEE}(T+S)_{i}\right]_{j}
$$

Most of the effort expended in the INTDF code is in calculating the integrated retentions of parent and daughter radionuclides in the source organs. Specific Effective Energies (SEE $(T+S)$ ) for Reference Man (ICRP 1975) were obtained from Oak Ridge National Laboratory (ORNL) on magnetic tape.

The number of transformations of a radionuclide in any organ or tissue of the body during any period of time is the time integral of activity of the radionuclide within that organ or tissue over the stated period of time, referred to as $U_{S}$ in the above equations. The function describing uptake and retention of a radionuclide in a body tissue following its ingestion or inhalation may be very complex. With certain exceptions (e.g., alkaline earth radionuclides in bone or iodine in the thyroid), the models used for this effort are based on the assumption that the body consists of a number of separate compartments. Any organ or tissue may comprise one or a number of compartments. Loss of the radionuclide from any compartment is assumed to be governed by first order kinetics. Therefore, the retention of any element in any organ or tissue will usually be described by either a single exponential term or the sum of a number of exponential terms. 
The compartment model used for the respiratory system is that of the ICRP Task Group on Lung Dynamics (ICRP 1966). The Task Group Lung Model (TGLM) has been slightly revised by Johnson and Carver (1981) to simplify the differential equations describing the retention of material in the tracheobronchial compartment, which is the formulation currently used.

The compartment model of the gastrointestinal tract (GI tract) is based on the model developed by Eve (1966). This is the same model used by ICRP 30 (1979-1982) and Johnson and Carver (1981). The GI tract is assumed to consist of four sections, the stomach, small intestine, upper large intestine, and lower large intestine.

After a radionuclide has been inhaled or ingested it will be translocated to the body fluids at a rate determined by the rate constants for the different compartments in the respiratory and gastrointestinal systems and by the radioactive decay constant of the radionuclide. Its translocation thereafter to the compartments representing the various organs and tissues of the body is represented in the model by a transfer compartment, which is assumed to be cleared by first order kinetics with a half time of 6 hours, unless specifically given a different rate constant in the metabolic data. Transformations occurring in the transfer compartment are assumed to be uniformly distributed throughout the body.

\subsection{DITTY}

The codes described above are intended to consider the environmental consequences in the near term (approximately 0 to 100 years) following releases to the environment. Such programs are useful for most nuclear fuel cycle facilities, except nuclear waste disposal sites, which may have longterm impacts. The long transport times through waste barriers and ground water aquifers require that longer time periods be considered. The computer code DITTY was developed to calculate the total population exposure over long time periods (Napier, Peloquin, and Strenge 1986). This code was incorporated, with only minor revisions, into the GENII package to provide the necessary capability to evaluate long-term releases.

Total population exposure is dependent on many parameters that are subject to large variations over thousands of years. Therefore, the user must 
realize that the calculated results from use of DITTY are only estimates and are limited in accuracy by the validity of the input assumptions. The time variant input parameters include 1) air and water source terms, 2) atmospheric dispersion patterns, and 3) exposed population.

Source terms of DITTY may be defined for releases to the atmosphere or to water. Releases to water may be to wells or surface water via ground water. The actual release rates are specified in an input file as the curies per year released for selected years following time zero for the calculation. One such data set is defined for each radionuclide of interest. Sophisticated ground water transport programs are generally used to determine the time variant release rate following transport from a geologic repository to the environmental access point (well or surface water). A similar definition of release rate as a function of time is needed for atmospheric releases.

For releases to the atmosphere, estimates of dispersion patterns are needed to determine total exposure of the regional population. The purpose of the dispersion calculation is to provide estimates of ground-level air concentrations of released radioactivity as a function of distance and direction from the release location. The downiwind air concentrations are related back to the release rate by use of "dispersion factors," which may be either supplied as input to the program or calculated by the program. When the program calculates the dispersion factors, meteorological data must be provided in the form of joint frequency of occurrence of windspeed, wind direction, and atmospheric stability. The dispersion calculation may be performed external to the program with the results entered through input, or joint frequency meteorological data may be supplied and dispersion factors calculated as a function of distance and direction. A straight-line crosswindaveraged Gaussian plume model is used for the dispersion calculation.

The regional population is defined for airborne and waterborne pathways as a function of time. For airborne pathways, the population is defined as a function of distance and direction corresponding to the locations for which dispersion factors are given or calculated. A population-weighted dispersion factor is calculated as the sum of the product of population and dispersion factor for each location. Alternatively, the population-weighted factor may be calculated external to the program and supplied as input. 
The time frame for the calculation is any 10,000-year period. This period is broken into 143 periods of 70 years each. The average release in each of these periods is calculated from source-term data provided, and the total population dose to selected organs is determined for each period, as illustrated in Figure 3.7. The activity present during any period is the sum of material released during that period (uniformly released over 70 years) and residual material in the environment from releases in previous periods. The dose is calculated for all contributing pathways of exposure, including external exposure, inhalation, and ingestion of contaminated water and foods. 


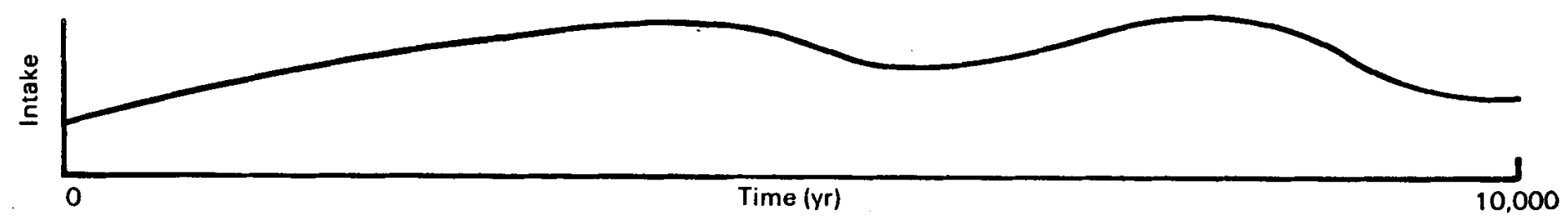

$\omega$
$\dot{v}$

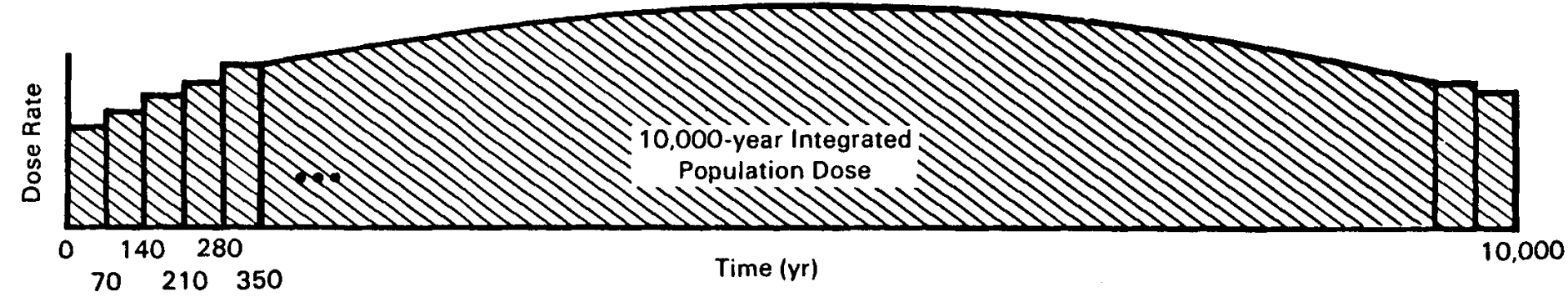

FIGURE 3.7. Calculational Scheme for Long-Term Integrated Population Doses 


\subsection{MATHEMATICAL REPRESENTATIONS}

Section 4.0 describes the mathematical models and algorithms used in the GENII package. It is divided into two broad sections: the equations used in the short-term analyses (i.e., those for the core GENII package) and the equations used in the long-term analyses (those for the DITTY code). The first six subsections deal with the core GENII modules of internal dosimetry, external dosimetry, atmospheric dispersion, air submersion, aquatic pathways, and terrestrial pathways. The seventh subsection is, to some extent, a restating of the previous subsections in the nomenclature of the long-term calculation.

\subsection{INTERNAL DOSIMETRY}

For this work, estimates are made of the dose equivalents in a number of target organs from the activity in a given source organ. For each type of radiation $i$, the dose equivalent $H_{i}$ in target organ $T$ from radionuclide $j$ in source organ $S$ is the product of two factiors:

- the total number of nuclear transformations of radionuclide $j$ in organ $S$ over the period of interest following intake

- the energy absorbed per gram in target organ $T$, suitably modified for the quality factor, from radiation of type $i$ per transformation of radionuclide $\mathrm{j}$ in source organ $\mathrm{S}$.

The number of nuclear transformations of the radionuclide in the body is a critical factor in the dose estimate. In order to implement the ICRP 30 type of calculations (ICRP 1979-1982), we have developed the INTDF code to solve this initial-value problem by means of a coupled set of differential equations.

\subsubsection{The General Model}

The complete compartment model or the general model used in most cases is illustrated in Figure 4.1. The TGLM lung model (ICRP 1966) and the Eve GI-tract model (Eve 1966) are connected to the transfer compartment. Each organ or tissue of deposition is assumed to consist of from one to four compartments, and from each of these compartments the radionuclide is 


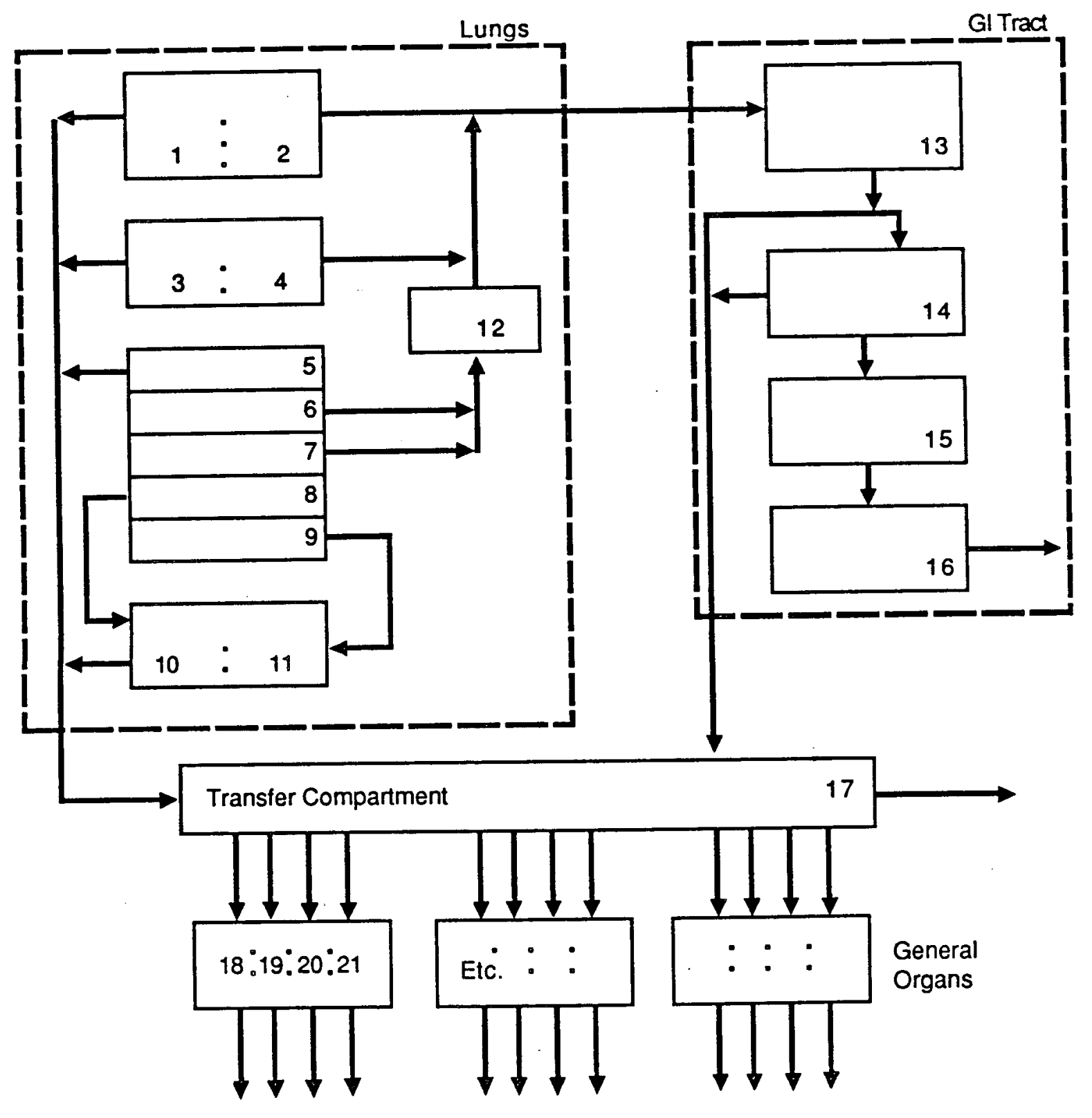

FIGURE 4.1. The General Model 
translocated at an appropriate rate to the excretion pathways. For simplicity, it is assumed in the general model that no feedback to the transfer compartment occurs either from the routes of excretion or from the organ compartments. Special models for which this is not the case are described as in the following subsections.

The differential equations that govern the transport of materials through the compartments illustrated in Figure 4.1 are listed in Table 4.1. The parameters used in Table 4.1 are defined as:

$Y_{i j}=$ the quantity of radionuclide $j$ in compartment $i$ as a function of time, Bq. For acute intakes, the model may be initialized with amounts of radionuclide $j$ in compartments 1 to 9 and/or 13 .

$I(t)=$ the intake rate of radionuclide $j$ into the body via inhalation (for equations for compartments 1 to 9 ) or ingestion (equation for compartment 13), Bq/day

$D_{j}=$ the deposition fraction of inhaled radionuclides into the major physiological portions of the lung, dependent on the particle size. The deposition fractions used correspond to a set of equations fit to the curves in Figure 5.1 of ICRP 30 , Part 1 (ICRP 1979a).

$F_{j}=$ the fraction of deposited material assumed to reside in each of the lung compartments, dependent on the translocation class of the inhaled material. Values of $F_{j}$ and $D_{j}$ are given in Table 4.2.

$\lambda_{j}=$ the rate constant describing the transfer of material out of compartment $i$, day -1

$\Delta_{i j}=$ the net rate of radiological decay (or ingrowth) for radionuclide $j$ in compartment $i, B q /$ day

$\delta_{f}=$ a Dirac delta function, where $\delta_{f}=1.0$ if $f_{1}$ is not equal to 1.0 , and where $\delta_{f}=0.0$ if $f_{1}=1.0$

$f_{1}=$ the fractional uptake from the GI tract to the body 
TABLE 4.1. Equations for the General Model Using Parameters Defined on Page 4.3

1. Lung Comparments

$$
\begin{aligned}
& \frac{d Y_{i j}}{d t}=I(t) D_{i} F_{i}-\lambda_{i} Y_{i j}-\Delta_{i j} ; i=1,9 \\
& \frac{d Y_{10 j}}{d t}=\lambda_{8} Y_{i j}-\lambda_{10} Y_{10 j}-\Delta_{10 j} \\
& \frac{d Y_{11 j}}{d t}=\lambda_{9} Y_{9 j}-\Delta_{10 j} \\
& \frac{d Y_{12 j}}{d t}=\lambda_{6} Y_{6 j}-\lambda_{7} Y_{7 j}-\lambda_{12} Y_{12 j}-\Delta_{12 j}
\end{aligned}
$$

2. GI-Tract Compartments

$$
\begin{aligned}
& \frac{d Y_{13 j}}{d t}=\left(\lambda_{2} Y_{2 j}+\lambda_{4}+\lambda_{4 j}+\lambda_{12} Y_{12 j}\right)+I(t)-\lambda_{13} Y_{13 j}-\Delta_{13 j} \\
& \frac{d Y_{14 j}}{d t}=\delta_{f} \lambda_{13} Y_{13 j}-\frac{\delta_{f} f_{1} \lambda_{14}}{1-f_{1}}+\lambda_{14} Y_{14 j}-\Delta_{14 j} \\
& \text { where } \quad \delta_{f}=1 \text { if } f_{1} \neq 1.0 \\
& \quad \delta_{f}=0 \text { if } f_{1}=1.0 \\
& \frac{d Y_{i j}}{d t}=\lambda_{i-1} Y_{i-1, j}-\lambda_{i} Y_{i j}-\Delta_{i j} i i=15,16
\end{aligned}
$$

3. Transfer Compartment

$$
\begin{aligned}
\frac{d Y_{17 j}}{d t}= & {\left[\lambda_{1} Y_{1 j}+\lambda_{3} Y_{3 j}+\lambda_{5} Y_{5 j}+\lambda_{10} Y_{10 j}\right] } \\
& +\left[\left(\frac{\delta_{f} f_{1} \lambda_{14}}{1-f}\right) Y_{14 j}+\left(1-\delta_{f}\right) \lambda_{13} Y_{13 j}\right]-\Delta_{17} Y_{17 j}-\Delta_{17 j}
\end{aligned}
$$

4. Organ Compartments

$$
\frac{d Y_{i j}}{d t}=f_{i} \lambda_{17} Y_{17 j}-\lambda_{i} Y_{i j}-\Delta_{i j} ; i=18,33
$$


IABLE 4.2. Deposition Fractions and Clearance Half Times $(a)\left(t_{1 / 2}\right)$ for the Lung Model

\begin{tabular}{|c|c|c|c|c|c|c|c|c|}
\hline \multirow[b]{2}{*}{ Region } & \multirow{2}{*}{$D_{i}{ }^{(b)}$} & \multirow{2}{*}{$\begin{array}{c}\text { Compart- } \\
\text { ment }\end{array}$} & \multicolumn{2}{|c|}{ Class D } & \multicolumn{2}{|c|}{ Class $\mathrm{W}$} & \multicolumn{2}{|c|}{ Class $Y$} \\
\hline & & & $F_{i}$ & $t_{1 / 2}$ & $F_{i}$ & $t_{1 / 2}$ & $F_{i}$ & $t_{1 / 2}$ \\
\hline $\begin{array}{l}\text { Naso- } \\
\text { Pharangeal }\end{array}$ & 0.3 & $\begin{array}{l}1 \\
2\end{array}$ & $\begin{array}{l}0.5 \\
0.5\end{array}$ & 0.01 & $\begin{array}{l}0.1 \\
0.9\end{array}$ & $\begin{array}{l}0.01 \\
0.4\end{array}$ & $\begin{array}{l}0.01 \\
0.99\end{array}$ & $\begin{array}{l}0.01 \\
0.4\end{array}$ \\
\hline $\begin{array}{l}\text { Tracheo- } \\
\text { Bronchial }\end{array}$ & 0.08 & $\begin{array}{l}3 \\
4\end{array}$ & $\begin{array}{l}0.95 \\
0.05\end{array}$ & $\begin{array}{l}0.01 \\
0 . ?\end{array}$ & $\begin{array}{l}0.5 \\
0.5\end{array}$ & $\begin{array}{l}0.01 \\
0.2\end{array}$ & $\begin{array}{l}0.01 \\
0.99\end{array}$ & $\begin{array}{l}0.01 \\
0.2\end{array}$ \\
\hline Pulmonary & 0.25 & $\begin{array}{l}5 \\
6 \\
7 \\
8 \\
9\end{array}$ & $\begin{array}{l}0.8 \\
0 \\
0 \\
0.2 \\
0\end{array}$ & $\begin{array}{l}0.15 \\
0 \\
0 \\
0.5 \\
0\end{array}$ & $\begin{array}{l}0.15 \\
0.4 \\
0.4 \\
0.05 \\
0\end{array}$ & $\begin{array}{r}50 \\
1 \\
50 \\
50 \\
0\end{array}$ & $\begin{array}{l}0.05 \\
0.4 \\
0.4 \\
0.135 \\
0.015\end{array}$ & $\begin{array}{r}500 \\
1 \\
500 \\
500 \\
500\end{array}$ \\
\hline Lymph & & $\begin{array}{l}10 \\
11\end{array}$ & $\begin{array}{l}0 \\
0\end{array}$ & $\begin{array}{l}0.5 \\
0\end{array}$ & $\begin{array}{l}0 \\
0\end{array}$ & $\begin{array}{r}50 \\
0\end{array}$ & $\begin{array}{l}0 \\
0\end{array}$ & $\begin{array}{c}1000 \\
\infty\end{array}$ \\
\hline
\end{tabular}

(a) Modified from ICRP 19 (1972) in days.

(b) For a 1- $\mu \mathrm{m}$-Activity Median Aerodynamic Diameter (AMAD) aerosol.

The term $\Delta_{i j}$ is developed using a recursive application of the Bateman equations (Bateman 1910), and the technique is further described in the following section on decay. However, it is used with the standard assumption that daughters produced in vivo can be described with the same metabolic parameters as the parent radionuclide. While this assumption is in general not valid, data in most cases do not exist from which the parameters for in vivo produced daughters can be estimated. Parameters can be estimated for the daughters not produced in vivo, but in general the daughters are transported to different organs than the parent. Even if the parent and daughter go to the same organ, the parameters describing the retention of the daughter taken up by the organ rather than produced in the organ are not necessarily the same.

Because the rates of excretion of the radionuclides from the body were not part of this investigation, the equations are not included that describe the elimination of material leaving the designated organs. 


\subsubsection{The Alkaline Earth Model}

The alkaline earth model implemented in ICRP 30 (1979-1982) calculations is based on the power function representation given by ICRP 20 (1973). The preferred model was the recycling model of Johnson (1981b). This model uses extra compartments in mineral bone to replace the power functions and includes recycling of materials through the transfer compartment. This model is illustrated schematically in Figure 4.2.

The differential equations describing the lung and GI-tract models used with the alkaline earth model are the same as those used for the general model. The differential equations describing the unique portions of the alkaline earth model are given in Table 4.3. This model replaces the general organ model of the general model with specific compartments for cancellous (trabecular) bone, cortical (compact) bone, bone surfaces, and other soft tissues. Because these compartments include inward as well as outward transfers, the notation of the equations in Table 4.3 includes $\lambda$ to be transfers into a compartment, and $\lambda^{\prime}$ to be transfer rates out of a compartment. 


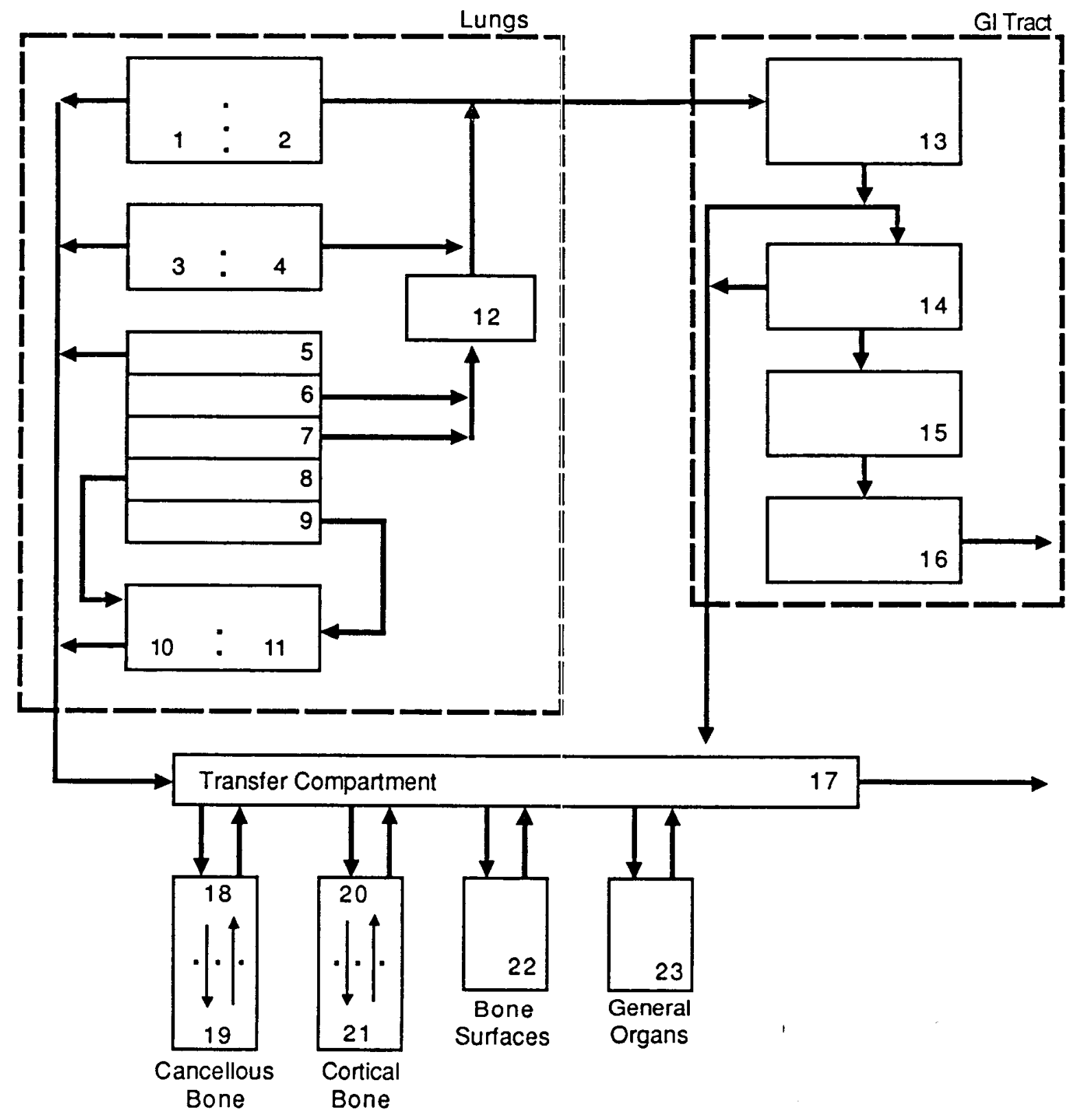

FIGURE 4.2. The Alkaline Earth Model 
TABLE 4.3. Equations for the Alkaline Earth Model

1. and 2. Include Lung and GI-Tract Compartments Identical to Those of General Model

3. Transfer Compartment

$$
\begin{aligned}
\frac{d Y_{17 j}}{d t}= & {\left[\lambda_{1} Y_{1 j}+\lambda_{3} Y_{3 j}+\lambda_{5} Y_{5 j}+\lambda_{10} Y_{10 j}\right] } \\
& +\frac{\delta_{f} f_{1} \lambda_{14}}{1-f_{1}} Y_{14 j}+\left(1-\delta_{f}\right) \lambda_{13} Y_{13 j} \\
& +\lambda_{18}^{\prime} Y_{18 j}+\lambda_{20}^{\prime} Y_{20 j}+\lambda_{22}^{\prime} Y_{22 j}+\lambda_{23}^{\prime} Y_{23 j} \\
& -\left(\lambda_{17}+\lambda_{18}+\lambda_{20}+\lambda_{22}+\lambda_{23}\right) Y_{17}-\Delta_{17 j}
\end{aligned}
$$

4. Cancellous Bone Compartments

$$
\begin{aligned}
& \frac{d Y_{18 j}}{d t}=\lambda_{18} Y_{17 j}+\lambda_{19} Y_{19 j}-\left(\lambda_{18}+\lambda_{19}\right) Y_{18 j}-\Delta_{18 j} \\
& \frac{d Y_{19 j}}{d t}=\lambda_{19} \lambda_{18 j}-\lambda_{19} Y_{19 j}-\Delta_{19 j}
\end{aligned}
$$

5. Cortical Bone Compartments

$$
\begin{aligned}
& \frac{d Y_{20 j}}{d t}=\lambda_{20} Y_{17 j}+\lambda_{21}^{\prime} Y_{21 j}-\left(\lambda_{20}^{\prime}+\lambda_{21}\right) Y_{20 j}-\Delta_{20 j} \\
& \frac{d Y_{21 j}}{d t}=\lambda_{21} Y_{20 j}-\lambda_{21}^{\prime} Y_{21 j}-\Delta_{21 j}
\end{aligned}
$$

6. Bone Surface Compartment

$$
\frac{d Y_{22 j}}{d t}=\lambda_{22} Y_{17 j}-\lambda_{22}^{\prime} Y_{22 j}-\Delta_{22 j}
$$

7. Other Soft Tissue Compartment

$$
\frac{d Y_{23 j}}{d t}=\lambda_{23} Y_{17 j}-\lambda_{23}^{\prime} Y_{23 j}-\Delta_{23 j}
$$




\subsubsection{The Iodine Model}

The iodine model implemented is that: of Johnson (1982), which includes the provisions for calculating doses to fetal thyroid from iodine ingestion by the mother. The lung and GI-tract moclels are the same as those of the general model, and the model for the maternal thyroid is essentially the same as that used for ICRP 30 (1979-1982). The model is illustrated in Figure 4.3. The differential equations that implement the model are listed in Table 4.4. All of the terms used are as defined above, with the addition of the fractional transfer terms $f_{f}$, for fraction to fetal thyroid from the maternal inorganic compartment, and $f_{m}$, for the fractional transfer to the maternal thyroid. The compartment for bladder is not used in the ICRP 30 model but is retained from Johnson in this implementation. The metabolic parameters for the thyroid are programmed into the subroutine, rather than read from the more general data library. These parameters are basically those of Johnson (1982), with the biological half time of iodine in the thyroid corrected to 80 days, as was actually used in ICRP 30 (see the errata to ICRP 30 Part 1 given at the end of ICRP 30 Part 3, 1981).

All decay products of the iodine as assumed to be stable or to escape the body before decaying (isotopes of the noble gas xenon).

The fetal thyroid model is included in the GENII system for future uses. The dose factors are not included in the routine tables of dose factor files normally accessed by the DOSE routine.

\subsubsection{The Tellurium/Lodine Model}

Some isotopes of tellurium decay to yield radioactive isotopes of iodine. It is assumed that all iodine produced in the body by the decay of tellurium is translocated instantaneously to the iodine inorganic transfer compartment. The metabolic behavior of iodine entering the inorganic transfer compartment this way is assumed to be governed by the model described above for iodine. The metabolic behavior of parent and daughter tellurium is governed by the general model.

The tellurium/iodine model is illustrated in Figure 4.4. The basic model is that of the general model, with the relevant portions of the iodine model included for iodine produced in the body. The equations implementing 


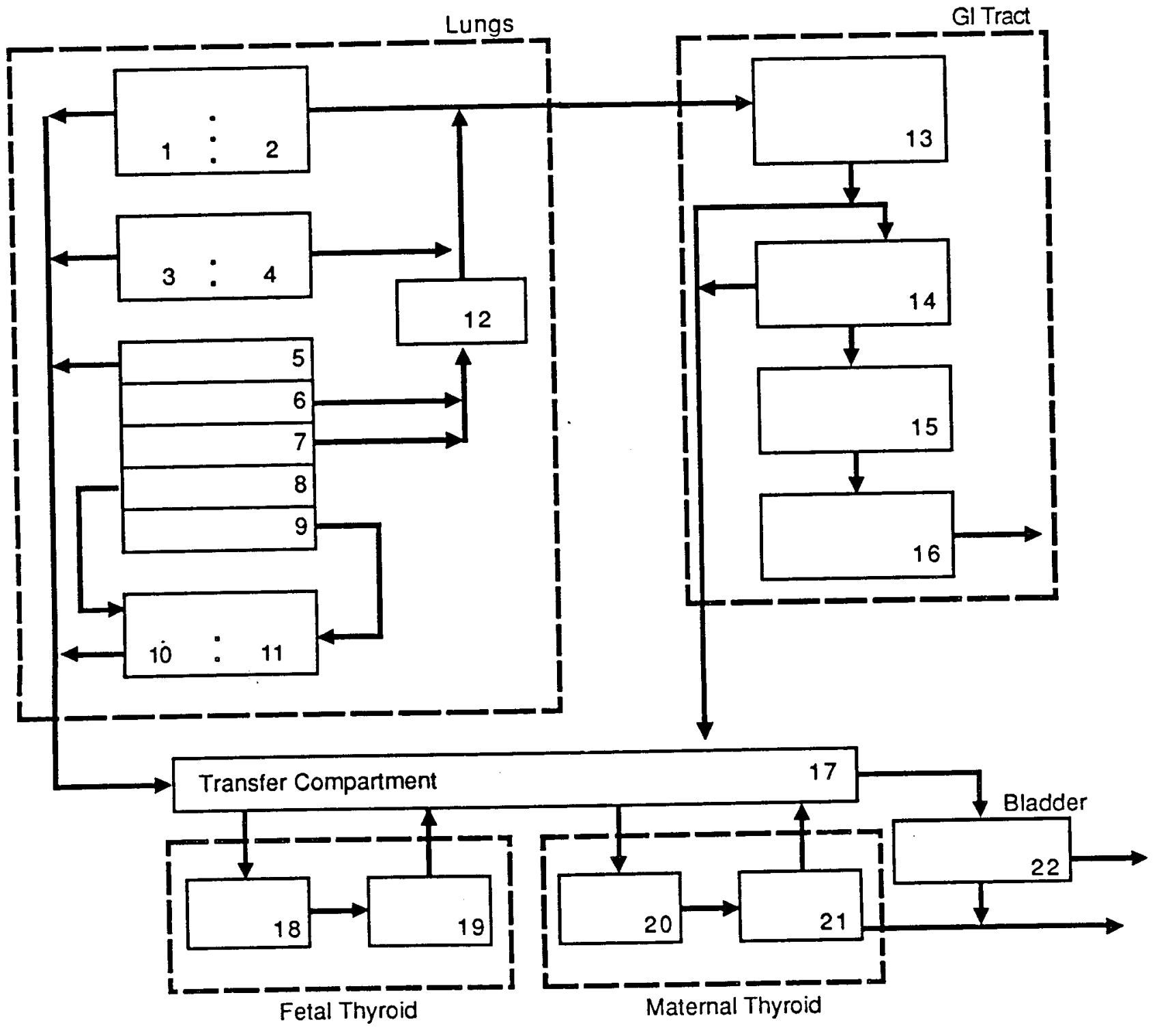

FIGURE 4.3. The Iodine Model 
IABLE 4.4. Equations for the Iodine Model

1. and 2. Include Lung and GI-Tract Comfiartments Identical to Those of General Model

3. Transfer Compartment (Maternal Inorganic)

$$
\begin{aligned}
\frac{d Y_{17}}{d t}= & {\left[\lambda_{1} \lambda_{1 j}+\lambda_{3} Y_{3 j}+\lambda_{5} Y_{5 j}+\lambda_{10} Y_{10 j}\right]+\lambda_{13} Y_{13 j} } \\
& +\lambda_{19} Y_{19 j}+0.9 \lambda_{21} Y_{21 j}-\lambda_{17} Y_{17 j}-\Delta_{17 j}
\end{aligned}
$$

4. Fetal Organs (Thyroid and Organic Compartment)

$$
\begin{aligned}
& \frac{d Y_{18 j}}{d t}=f_{f} \lambda_{17} Y_{17 j}-\lambda_{18} Y_{18 j}-\Delta_{18 j} \\
& \frac{d Y_{19 j}}{d t}=\lambda_{18} Y_{18 j}-\lambda_{19} Y_{19 j}-\Delta_{19 j}
\end{aligned}
$$

5. Maternal Organs (Thyroid and Organic Compartment)

$$
\begin{aligned}
& \frac{d Y_{20 j}}{d t}=f_{m} \lambda_{17} Y_{17 j}-\lambda_{20} Y_{20 j}-\Delta_{20 j} \\
& \frac{d Y_{21 j}}{d t}=\lambda_{20} Y_{20 j}-\lambda_{21} Y_{21 j}-\Delta_{21 j}
\end{aligned}
$$

6. Bladder

$$
\begin{aligned}
& \frac{d Y_{22 j}}{d t}=\left(1-f_{f}-f_{m}\right) \lambda_{17} Y_{17 j}-0.1 * 0.9 * \lambda_{21} Y_{21 j}-\lambda_{22} Y_{22 j}-\Delta_{22 j} \\
& \text { where } f_{f}=0.0, \text { if age } \leq 90 \text { days or age }>270 \text { days } \\
& f_{f}=0.03(\text { age } / 90-1) \text {, if } 90<\text { age } \leq 270 \text { days } \\
& f_{m}=0.3
\end{aligned}
$$

7. Note: All xenon decay products are assumed to escape the body before decaying. 


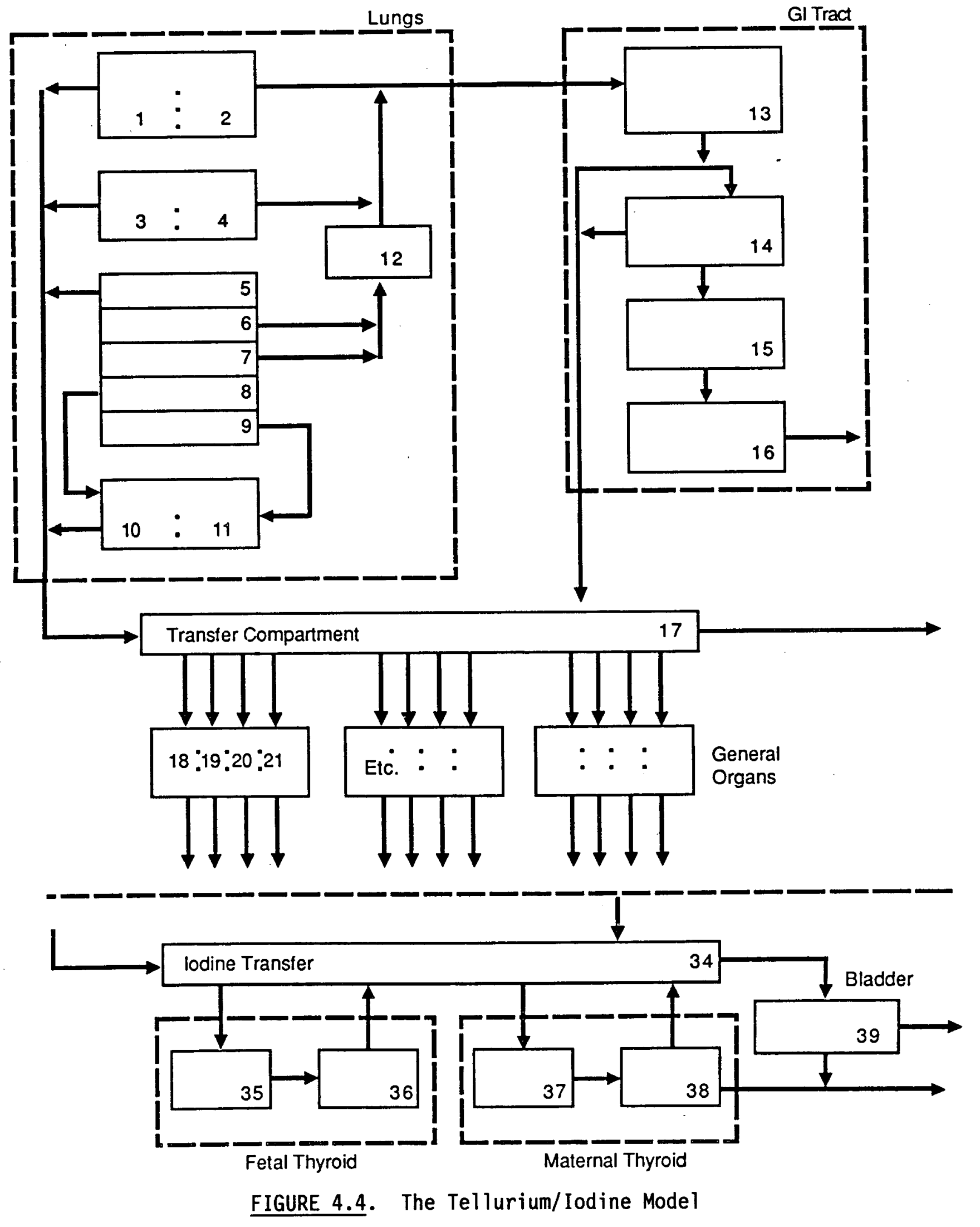

4.12 
this model are essentially identical to those for the other two models, with appropriate revision of the maternal inorganic compartment to indicate that in vivo produced iodine is all moved instantaneously to that compartment. This equation is given in Table 4.5. The combination of the tellurium/iodine model including the fetal thyroid dose calculation capability is unique in our experience.

As with the basic iodine model, all xenon decay products of the iodine are assumed to escape the body before decaying.

\subsubsection{The Radium/Radon Model}

A special case of the alkaline earth model is implemented for the decay of Ra-226 through Rn-222. For this decay chain, radon is assumed to diffuse entirely out of soft tissues of the body before decaying and to retain only $30 \%$ in mineral bone tissues (ICRP 1979a). Daughter products of the radon are allowed to continue to build into the bone compartments. The equations used to implement this option are provided in Table 4.6.

\subsubsection{The Tritium and Carbon Models}

Tritium and carbon-14 are radionuclides handled by the general model (Section 4.1.1) in a slightly modified marner. Both are elements that are integral to the human metabolism.

Tritium is assumed to be in the form of tritiated water. All tritiated water ingested or inhaled is assumed to be completely and instantaneously absorbed into the transfer compartment. Tritiated water is assumed to be uniformly distributed among the soft tissues at any time following intake, where it is retained with a biological half time of 10 days. In addition, exposure to an atmosphere containing tritium results in intake of that material by absorption through intact skin. The combined total rate of intake of tritium in air is assumed to be $150 \%$ of the inhalation intake rate alone.

In a similar fashion, all intakes of carbon-14 are assumed to be completely and instantaneously absorbed into the transfer compartment. This is equivalent to assuming that inhaled material is carbon dioxide gas and that ingested materials are readily absorbed carbohydrates. Carbon is assumed to be distributed throughout the organs and tissues of the body, where it is 


\section{TABLE 4.5. Equations for the Tellurium/Iodine Model}

1. All parent (tellurium) equations are identical to those of the general model.

2. Transfer Compartment (for iodine only) (Maternal Inorganic)

$$
\frac{d y_{34 j}}{d t}=\sum_{n} \Delta_{n j}+\lambda_{36} Y_{36 j}+0.9 \lambda_{38} Y_{38 j}-\lambda_{34} Y_{34 j}
$$

where $n=$ total of all organs and all parents yielding iodine $j=$ iodine index only.

3. All iodine transfers are governed as for iodine model (with the compartment indices as appropriate).

4. Note: A11 xenon decay products of iodine are assumed to escape the body before decaying.

\section{TABLE 4.6. Equations for the Radium/Radon Model}

1. All equations are identical to those of the alkaline earth model, with the exception of RN daughter handling.

2. Soft Tissue Compartments

$$
\Delta_{i j}=0.0 ; j=\text { RN222 index }
$$

3. Bone Compartments

$$
\lambda_{i j}^{\prime}=0.3 \Delta_{i j} ; j=\text { RN222 index }
$$

$\lambda_{i j}^{\prime}$ is then used throughout, replacing $\Delta_{i j}$ 
retained with a biological half time of 40 days. This assumption is considered by ICRP 30 (ICRP 1979-1982) to yield realistic estimates for carbon14-labeled metabolites and to overestimate doses from most other carbon-14labeled compounds.

\subsubsection{Decay Data}

Radioactive nuclei sometimes decay through a number of unstable daughters before reaching a stable (or very-long-lived) end state. These decay chains are important in that the decay energies of the daughter products contribute to the total dose received from intake of the parent. The data on radionuclide half-lives, decay chains, and various fractional branching ratios within chains are largely taken from the DRALIST data of Kocher (1981b) for consistency with gamma energy data used elsewhere in the GENII code package. These data are listed in a standard library accessed by the computer codes.

Radionuclides of half-life less than 10 minutes are of little importance in environmental calculations and in terms of internal dose, except where they are members of longer decay chains. All decay data in the standard library that is used represent radionuclides with half-lives greater than 10 minutes. For chains with daughters with very short half-lives, the decay energy associated with these short-lived daughters has been assigned to the parent. This assignment includes correction of the SEE factors in the dose calculations. The appropriate branching ratios have been considered.

The computer programs within the GENII package include a generalized chain decay processor. This processor can give the activity of any member of a decay chain as a function of time from any initializing condition. Variants of the processor provide the total activities of chain members for conditions of continual input of the parent to a system. The chain processor (ACHAIN, with variants BCHAIN, DGCHAIN, etc.) operates on a recursive application of the Bateman equations (Bateman 1910).

The Bateman equations provide a simple means of solving the differential equations describing chain decay. The basic form of the equation is

$$
A_{n}=N_{10}\left[c_{1} e^{-\lambda_{1} t}+c_{2} e^{-\lambda_{2} t}+\ldots+c_{n} e^{-\lambda_{n} t}\right]
$$




$$
c_{i}=\frac{\lambda_{1} \lambda_{2} \ldots \lambda_{i-1}}{\prod_{\substack{j=1 \\ j=i}}^{n}\left(\lambda_{j}-\lambda_{j}\right)}
$$

where $A_{n}=$ the activity of the nth daughter

$\mathrm{N}_{10}=$ the initial activity of the parent at time $t=0$

$\lambda_{j}=$ the decay constant for nuclide $i$ of the chain.

For initial conditions where initial concentrations of the daughter radionuclides exist, the equations can be applied assuming each nuclide is the start of a chain. The results are then summed.

Another variant in general decay schemes is branching decay. In this case, the Bateman solution is applied by using partial decay constants (that is, the decay constant times the branching fraction) in the numerator of the constants $c_{j}$. If the decay chain branches and subsequently the two branches are rejoined (as in the natural radioactive series), the two branches are treated by this method as separate chains. The production of a common member beyond the branch point is the sum of the two paths.

\subsubsection{Metabolic Data}

Values for the fractional uptake of materials, their transfer coefficients from compartment to compartment, and their elimination rates are all contained in a standard computer library. The basic source of this information is the computer code GENMOD. Most of the data in this library is adapted directly from ICRP 30 (1979-1982). It also contains the specialized parameters for the alkal ine earth model ( $\mathrm{Ca}, \mathrm{Sr}, \mathrm{Ba}, \mathrm{Ra})$ of Johnson and Myers (1981).

The parameters for the iodine model have been deleted from the library and hard-wired into the computer code. This simplifies both the iodine model and especially the tellurium/iodine model.

In some instances, the parameters obtained from GENMOD have reverted to those of the ICRP because no documentation of the values that came in the original file was available. It could not be determined if those values were 
intentionally used. (A specific example is metabolic data for technetium in thyroid.)

The actinide metabolic data have been updated to account for the recommendations of ICRP 48 (1986). This includes new $f_{1}$ factors, as well as the half-time data and fractional uptake data for specific organs.

The data adopted from GENMOD includes metabolic data for infants (up to one year old). This portion of the data has not as yet been extensively explored. The methods of deriving this information are given briefly in Johnson and Carver (1981) and seem reasonable, but the information has not yet been verified. The data may eventually be used with some minor variations on the standard transfer rates for the lung and GI-tract model.

\subsubsection{Dosimetric Data}

As described above, once the integrated retentions of the radionuclides in the source organs have been calculated, the dose to each target organ is calculated for each radiation of type $i$ from radionuclide $j$

$$
H(T+S)_{i}=U_{S} \cdot 1.6 \cdot 10-13 \mathrm{SEE}(T+S)_{j} \cdot 10^{3}
$$

where $\quad U_{s}=$ the number of transformations of radionuclide $j$ in source organ $S$ over the period of integration following intake of the radionuclide

$1.6 \cdot 10-13$ = the number of joules in $1 \mathrm{MeV}$

$\operatorname{SEE}(T+S)_{i}$ (in MeV $g^{-1}$ per transformation) $=$ the specific effective energy for radiation type $i$, suitaljy modified by quality factor, absorbed in $T$ from each transformation in $S$

$10^{3}=$ the conversion factor from $\mathrm{g}^{-1}$ to $\mathrm{kg}^{-1}$.

In general, for the intake of any mixture of radionuclides, i.e., parent with daughters, the dose in target organ $T$ from radioactivity in several different sources $S$ is given by

$$
H=1.6 \cdot 10^{-10} \sum_{S} \sum_{j}\left[U_{S} \sum_{i} \operatorname{SEE}(T+S)_{i}\right]_{j}
$$


The factors for specific effective energy are from a tape of SEE factors from ORNL.(a) For purposes of tabulating SEE values, the body is divided into a set of identified source and target regions with the remaining tissue assigned as "other". This region is largely muscle. The breast dose is based on the SEE values for "other" as a target.

The metabolic models used in the calculations partition the activity in the body among a number of organs and assign the residual to be uniformly distributed among the remaining tissues, generally referred to as the "other" tissue. This "other" tissue group does not necessarily correspond to that noted in the previous paragraph. Calculations of the number of nuclear transformations are performed for the lung, GI tract, transfer compartment, organs identified in the metabolic model, and the "other" tissue group (if it is identified in the model). The transformations in the transfer compartment are distributed among the "identified" organs and the "other" by mass fraction. The SEE value for "other" as a source is computed as

$$
\operatorname{SEE}(T+T B)=\frac{M_{T B} S E E(T+T B)-\sum_{S} M_{S} \operatorname{SEE}(T+S)}{M_{\text {OTHER }}}
$$

where MTB and MS are the masses of the total body and source organs. The summation extends over all explicitly identified organs (source regions) in the metabolic model. The mass of the "other" tissue is

$$
M_{\text {OTHER }}=M_{T B}-\sum_{S} M_{S}
$$

The computer code for calculating internal dose, INTDF, does not itself provide effective dose equivalent (whole body dose). Instead, it calculates the incremental organ doses for each year following an initial intake. The output is the incremental dose to each organ, which is then used in other portions of the code to assemble annual doses, dose commitments, and

(a) Personal communication from Keith Ackerman of Oak Ridge National Laboratory, Oak Ridge, Tennessee. 
accumulated doses. The method by which these doses are assembled was described in Section 3.4.

\subsection{EXTERNAL DOSIMETRY}

The ISOSHLD code that is the basis of the EXTDF module of GENII has been documented and verified numerous times (Engel, Greenborg, and Hendrickson 1966; Simmons et a1. 1967; Mansius 1969). It is a basic shielding code using the commonly accepted techniques of Rockwell (1956) or other standard references. Only the basic theory is reproduced here.

\subsubsection{Point Kernel Integration}

Figure 4.5 illustrates a point source of gamma-ray photons, So (in this example within a large cylindrical volume, SV), that emits with equal intensity in all directions.

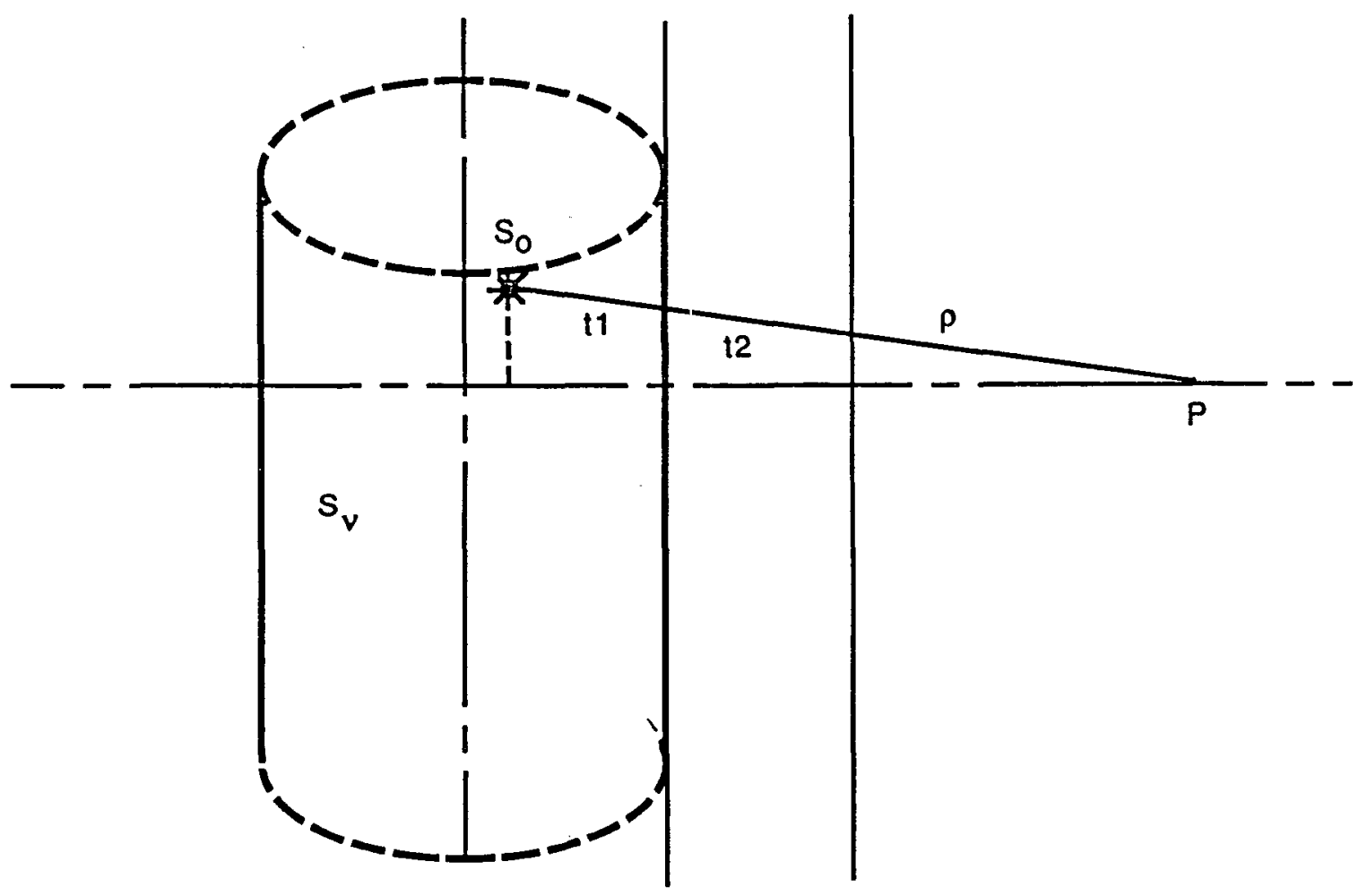

FIGURE 4.5. Point Source (So) Within a Finite Cylindrical Volume (Sv) at a Distance $p$ from Point $P$ Through Shields of Thickness t1 and t2 
The dose rate at point $P$ from photons originating at $S_{0}$ is given by

$$
D=\frac{S_{0} B e^{-b_{1}}}{K 4 \pi \rho^{2}}
$$

where $b_{1}=\sum_{i=1}^{N} \mu_{i} t_{i}$

$$
D=\text { dose rate, } R / h
$$

$S_{0}=$ emission rate of gamma rays, photons $/ \mathrm{sec}$

$B=$ build-up factor, dimensionless

$\mu_{i}=$ linear absorption coefficient of the $i$ th shield, $\mathrm{cm}^{-1}$

$t_{i}=$ slant distance through the $i$ th shield, $\mathrm{cm}$

$K=$ conversion of gamma-ray flux to dose rate

$\rho=$ distance from source to dose point, $\mathrm{cm}$

$\mathrm{N}=$ number of shields.

The dose rate at point $P$ from photons of all initial energies originating at all points within the source volume, SV, is given by

$$
D_{\text {tot }}=\int_{E} \int_{V} \frac{S_{0}(E, V) B\left(E, b_{1}\right) e^{-b_{1}}}{K(E) 4 \pi \rho^{2}(V)} d V d E
$$

Equation (4.2.2) may be analytically integrated for a few simple geometries, and this technique is used to obtain some of the extended source dose formulas given in the shielding handbooks (Rockwell 1956; Etherington 1958; Blizard 1962). This equation may be numerically integrated for virtually any source geometry; however, only regular geometric shapes are normally considered suitable for computer integration. Complex geometric shapes are generally constructed by synthesis of simple shapes.

This numerical integration technique (commonly referred to as point kernel integration) divides the source volume into a number of differential volumes. The source energy is divided into a number of energy groups narrow enough to consider the build-up factors, attenuation coefficients, and dose conversion factors consistent over the energy range of the group. Each monoenergetic differential volume source is then treated as a point source, and 
the dose from each of these point sources is calculated. For each point source, new values of $B, \mu_{i}, t_{i}, \rho, S_{0}, b_{l}$, and $k$ are needed and are calculated using trigonometric relationships and basic data appropriate to the system geometry, source photon energies, and materials. Integration over source volume and source energy is then obtained by summing the dose contributions from all differential source volumes over all source energies.

\subsubsection{Mixed Mass Attenuation Coefficients}

Linked to EXTDF is a library of mass attenuation coefficients in 16 energy groups for 20 common materials. These materials are water, tissue, air, hydrogen, lithium, carbon, aluminum, titanium, iron, nickel, zirconium, tin, tungsten, lead, uranium, ordinary and magnetite concretes, strontium, promethium, and cerium. The attenuation coefficients are listed for materials of unit density. For each source ancl shield region, the code input requires the library material number and the material density. Up to five materials per region are allowed. Thus, EXTDF calculates region mixed mass attenuation coefficients, $\mu_{i}$, for each recion, $i$, by

$$
\mu_{i}=\sum_{j} d_{j} \mu_{j}
$$

where $j$ is the index of materials specified to be in region $i$. If a combination of the previous 10 materials will not adequately describe a desired shield material, the code user may add additional materials to this library.

\subsubsection{The Build-Up Factor}

Linked to EXTDF is a build-up factor library that contains, for 16 photon energies, the coefficients $A, a_{1}$, and $a_{2}$ of Taylor's equation (Rockwel1 1986)

$$
B=A e^{-a_{1} b_{1}}+(1-A) e^{-a_{2} b_{1}}
$$

for dose build-up from a point isotropic source in seven materials: water, aluminum, iron, tin, tungsten, lead, and uranium. This material range covers the effective atomic numbers (EANs) from 4 to 92 . The data in this library were taken from Goldstein (1959, p. 376). 
In the process of kernel integration, the build-up factor for each differential source volume is calculated for all of the materials between source point and dose point. Values of $t_{i}$, the source point to dose point line of sight distance through the source and shield regions, are calculated for each new source point by trigonometric relationships. Values of $\mu_{1}$ for each shield region are obtained from Equation (4.2.3). EXTDF then calculates $b_{1}$ for the line of sight from the source point to dose point, and $b_{1}$ is used in Taylor's equation to obtain the correct build-up, B.

Several considerations are discussed here in regard to how $A_{1} \alpha_{1}$, and $\alpha_{2}$ (from the build-up factor library) are used. The method of selection is by effective atomic number. The EAN has been defined in a number of ways (discussed in Murty 1965); however, within EXTDF it is defined as

$$
E A N=\frac{\sum_{j} \frac{d_{j} Z_{j}}{A_{j}}}{\sum_{j} \frac{d_{j}}{A_{j}}}
$$

where $Z_{j}=$ atomic number of species $j$ in the shield

$A_{j}=$ atomic weight of species $j$ in the shield

$d_{j}=$ density of species $j$ in the shield.

A particular shield region should be chosen whose characteristics will be used in calculating build-up through all shields in the system. This region is usually the last shield region, provided the region is thick compared to the sum thickness of the other regions. If no build-up region choice is made, the code will use the last defined region. The EAN is then calculated for the chosen region. EXTDF brackets this effective atomic number in the build-up factor table, calculates the build-up factors for both effective atomic numbers available in the library, and then interpolates to obtain the final build-up factor for use in the numerical integration.

Because the atomic weight for each material in the library is required for use in evaluating EAN, an effective atomic weight (EAW) was defined for the mixed elemental materials given in the library. These were calculated by 


$$
E A W=\frac{\sum_{k} N_{k} A_{k}}{\sum_{k} N_{k}}
$$

where $N_{k}$ is atomic density of species $K$ in the mix, and $A_{k}$ is the atomic weight of species $k$ in the mix.

In summary, the build-up treatment in EXTDF includes all mean free paths in material between the source point and dose point and has the build-up characteristics of the last (or a particular specified shield region).

\subsubsection{Conversion of Surface Dose to Effective Dose Equivalent}

External dose rate calculations traditionally end at an exposure rate in air, in $\mathrm{R} / \mathrm{hr}$ or equivalent units. The EXTDF results are used in conjunction with internal dose factors expressed in terms of effective dose equivalent, i.e., the weighted sum of organ doses. Therefore, all results are converted to effective dose equivalent using energy-dependent surface-dose to organdose conversion factors derived from information in Kocher (1981a). These values were derived from dose-rate factors for monoenergetic sources at the body surface given by $0^{\prime} B r i e n$ and Sanna (1976) and from scattered spectra for monoenergetic sources in an infinite atmospheric cloud given by Dillman (1974); the calculations were performed by Eckerman and Kerr in Kocher (1981a). The results are comparable to, but slightly larger than, those given by Kocher (1979) and Till and Meyer (1983). For GENII, these were weighted with the ICRP 26 (1977) organ weighting factors and summed to give the surface-dose to effective dose equivalent conversion factors listed in Table 4.7 . 
TABLE 4.7. Photon Energy Group Definitions Conversion Factors Used in the EXTDF Code

\begin{tabular}{ccccc} 
Group & $\begin{array}{c}\text { Photon Energy } \\
\text { Range, MeV }\end{array}$ & $\begin{array}{c}\text { Average Photon } \\
\text { Energy, MeV }\end{array}$ & $\begin{array}{c}\text { Surface Dose to } \\
\text { EDE Factor (rem/rad) }\end{array}$ \\
\cline { 2 - 2 } 1 & $0.01-0.02$ & 0.015 & 0.0424 \\
2 & $0.02-0.03$ & 0.025 & 0.131 \\
3 & $0.03-0.04$ & 0.035 & 0.267 \\
4 & $0.04-0.05$ & 0.045 & 0.410 \\
5 & $0.05-0.06$ & 0.055 & 0.507 \\
6 & $0.06-0.07$ & 0.065 & 0.550 \\
7 & $0.07-0.08$ & 0.075 & 0.59 \\
8 & $0.08-0.09$ & 0.085 & 0.62 \\
9 & $0.09-0.1$ & 0.095 & 0.65 \\
10 & $0.1-0.2$ & 0.15 & 0.67 \\
11 & $0.2-0.3$ & 0.25 & 0.66 \\
12 & $0.3-0.4$ & 0.35 & 0.65 \\
13 & $0.4-0.55$ & 0.475 & 0.65 \\
14 & $0.55-0.75$ & 0.65 & 0.66 \\
15 & $0.75-0.9$ & 0.825 & 0.67 \\
16 & $0.9-1.1$ & 1.0 & 0.68 \\
17 & $1.1-1.35$ & 1.225 & 0.7 \\
18 & $1.35-1.6$ & 1.475 & 0.72 \\
19 & $1.6-1.8$ & 1.7 & 0.74 \\
20 & $1.8-2.0$ & 1.9 & 0.76 \\
21 & $2.0-2.2$ & 2.1 & 0.765 \\
22 & $2.2-2.4$ & 2.3 & 0.77 \\
23 & $2.4-2.6$ & 2.5 & 0.775 \\
24 & $2.6-2.8$ & 2.7 & 0.777 \\
25 & $2.8-3.2$ & 3.0 & 0.78 \\
& & &
\end{tabular}




\subsection{ATMOSPHERIC DISPERSION}

The dose to a receptor from an atmospheric release depends on the spatial and temporal distribution of radionuclides in the environment. This distribution is a function of the way in which the material is released and of the environment between the release point and the receptor. Atmospheric dispersion models describe the transport and diffusion of material released to the atmosphere. They may also describe the depletion of material as a result of deposition on surfaces, washout by precipitation, chemical transformations, and radioactive decay. Kao's report (1984) contains mathematical derivations of several general diffusion models.

Selection of a dispersion model for a specific application should be based on intended model use, source characteristics, and available meteorological data. Simple atmospheric models are appropriate for making concentration estimates to be used in evaluating potential doses for environmental impact statements and preliminary safety analyses because the releases are hypothetical, the receptors are not in the immediate vicinity of the release point, and the evaluation is based on assumed meteorological conditions. The simple models may also be appropriate for assessing the consequences of a routine release if the release is constant for prolonged periods and if no systematic spatial variations of the metecorological conditions occur. More complex models are required where topography has a significant effect on atmospheric transport and diffusion. In addition, complex models should be used for evaluating the consequences of accidental releases where actual meteorological data are available. Specialized models are required if concentration estimates are needed in the immediate vicinity of a release point. These specialized models require specific information about the geometry of the release because they must treat initial plume conditions. Briggs (1984) and Hosker (1984) discuss problems associated with modeling transport and diffusion near the source.

Simple atmospheric dispersion models are appropriate for estimating radiological doses from potential routine and accidental radionuclide releases at Hanford. For cases where simple models are not appropriate, the GENII package allows input or realistic data calculated independently of the 
GENII models. GENII also includes an empirical building wake diffusion model that may be used to make concentration estimates near the source.

The GENII package implements a selection of straight-line Gaussian-plume models for use when appropriate. These models are used to estimate air concentrations on a grid for 16 directions and up to 10 distances. The GENII models make use of this information, along with information on individual location or population distribution, to project exposures from atmospheric releases.

\subsubsection{Gaussian Plume Models}

The straight-line Gaussian plume model is the basis of a set of dispersion models that are widely accepted for routine dose assessment applications. In a strict sense, these models are appropriate if:

1) the release is from a source that has dimensions that are small compared to the distances at which concentrations are to be estimated

2) the terrain is relatively flat and has uniform surface conditions in all directions

3) the atmospheric conditions at the time and location of the release completely control the transport and diffusion of material in the atmosphere.

It is not appropriate to apply these models to releases in valleys, near lake shores, or along coasts because they may produce unrealistic dose estimates. The basic Gaussian plume model for a continuous release is

$$
\bar{\chi}=\frac{Q^{\prime}}{2 \pi \sigma_{y} \sigma_{z} U} F(y) G(z)
$$

where $\quad \bar{\chi}=$ the concentration at the receptor $\left(\mathrm{Ci} / \mathrm{m}^{3}\right)$

$Q^{\prime}=$ the release rate $(\mathrm{Ci} / \mathrm{sec})$

$\sigma_{y}=$ the horizontal diffusion coefficient (m)

$\sigma_{z}=$ the vertical diffusion coefficient (m)

$U=$ the average wind speed $(\mathrm{m} / \mathrm{sec})$

$F(y)=$ a horizontal off-axis correction term 
$G(z)=$ a vertical off-axis correction term.

In this form, the $x$ axis is assumed to be directed in the downwind (transport) direction, the $y$ axis is directed to the left, and the $z$ axis is directed vertically upward. The diffusion coefficients describe the spread of the plume and are functions of the atmospheric conditions and the time that it takes material to be transported from the release point to the receptor. In addition, they are frequently given as functions of atmospheric stability and distance from the source. Farameterization of diffusion coefficients is discussed in Section 4.3.2. Initial dilution of material in the plume is determined by the wind speed at the height of the release. If the release takes place at or near ground level, it is common practice to calculate the wind speed at $10 \mathrm{~m}$ above ground in computations.

The horizontal and vertical off-axis correction terms are dimensionless quantities that describe the decrease in concentration with increasing distance from the plume axis. The horizontal correction term has the form

$$
F(y)=\exp \left[-0.5\left(y / \sigma_{y}{ }^{2}\right]\right.
$$

where $y$ is the horizontal distance to the plume axis in meters. At the plume axis, $y=0$ and $F(y)=1$. As the distance from the axis increases, $F(y)$ decreases in the familiar bell-shaped form that is characteristic of Gaussian curves. The initial form of the vertical off-axis correction term is similar to that given for $F(y)$. It is

$$
G(z)=\exp \left\{-0.5\left[\left(z-h_{e}\right) / \sigma_{z}\right]^{2}\right\}
$$

where $z$ is the height of the receptor above ground in meters, and $h_{e}$ is the effective release height in meters. The effective release height is the actual release height corrected as appropriate for initial plume rise or downwash. Estimating the effective release height is discussed in Section 4.3.3. Vertical diffusion is limited by the ground at the bottom of the atmosphere. Upward diffusion may also be limited. In this situation, the limit is called the top of the mixing layer. 
The ground and the top of the mixing layer are generally assumed to reflect material. These characteristics are incorporated into $G(z)$ by use of virtual sources below the ground and above the top of the mixing layer (e.g., see Csanady 1973). The resulting form of $G(z)$ is

$$
\begin{aligned}
G(z)= & \sum_{n=-\infty}^{\infty}\left\{\exp \left\{-0.5\left[\left(2 n H-h_{e}+z\right) / \sigma_{z}\right]^{2}\right\}\right. \\
& \left.+\exp \left\{-0.5\left[\left(2 n H-h_{e}-z\right) / \sigma_{z}\right]^{2}\right\}\right\}
\end{aligned}
$$

where $H$ is the mixing layer thickness (depth) in meters.

Several special cases of interest are included in this relationship. If source and receptor are at ground level and the mixing layer thickness is large compared to the vertical diffusion coefficient,

$$
G(z)=2.0
$$

For an elevated release and ground-level receptor, if the mixing layer thickness is large compared to the release height and the vertical diffusion coefficient, then

$$
G(z)=2.0 \exp \left[-0.5\left(h_{e} / \sigma_{z}\right)^{2}\right]
$$

If the receptor is elevated but the remaining conditions remain true, then

$$
G(z)=\exp \left\{-0.5\left[\left(h_{e}-z\right) / \sigma_{z}\right]^{2}\right\}+\exp \left\{-0.5\left[\left(h_{e}+z\right) / \sigma_{z}\right]^{2}\right\}
$$

Finally, if the receptor is at ground level, the release is elevated, and the thickness is not large, then

$$
G(z)=2 \sum_{n=-\infty}^{\infty} \exp \left\{-0.5\left[\left(2 n H-h_{e}\right) / \sigma_{z}\right]^{2}\right\}
$$


As the plume grows vertically, it gradually becomes uniformly mixed throughout the mixing layer. Uniform mixing is reached when the vertical diffusion coefficient reaches approximately $0.8 \mathrm{H}$. Beyond the distance at which this occurs, the average concentration in the plume is given by

$$
\bar{\chi}=\frac{Q^{\prime}}{(2 \pi)^{1 / 2} \sigma_{y} H U} F(y)
$$

The Gaussian models, as defined by Equations (4.3.1) and (4.3.9), are most appropriate for estimating average concentrations for periods that are longer than the time of travel from the source to receptor and not so long that atmospheric conditions are likely to change. This restricts limits application of Equations (4.3.1) and $(4.3 .9)$ to periods of less than 12 hours and probably 6 hours or less. As the duration of a release increases, it becomes less likely that the wind direction has remained constant since the beginning of the release. To compensate for meandering of the wind, it is assumed that the material in a plume is uniformly distributed across a sector $22.5^{\circ}$ in width ( $\pi / 8$ radians). With this assumption, the average concentration in a plume in which the vertical diffusion has not been limited becomes

$$
\bar{\chi}=\frac{4 \sqrt{2} Q^{\prime}}{\pi^{3 / 2} \times \sigma_{z} U} G(z)
$$

where $x$ is the distance between the source and receptor. In plumes with a uniform vertical concentration profile, the concentration becomes

$$
\bar{\chi}=\frac{8 Q^{\prime}}{\pi \times H U}
$$

If the release continues, it becomes necessary to consider the effects of changes in atmospheric stability, wind speed, and mixing layer thickness, as well as larger changes in wind direction. Equations (4.3.10) and (4.3.11) can estimate the concentration in the plume under each combination of 
conditions, but it is no longer appropriate to assume that a receptor is always within the plume. The concentration at a receptor must be estimated by averaging the concentrations during periods when individual plume segments affect the receptor and as well as during the periods when the plume is elsewhere. This long-term average is given by

$$
\bar{\chi}=\frac{4 \sqrt{2} Q^{\prime}}{\pi x} \sum_{j} \sum_{k} \sum_{T} \frac{G(z)}{\sigma_{z} U} P_{i j k 1}
$$

where $P_{i j k l}$ is the joint probability that the wind direction falls within class $i$ and speed falls within class $j$, that the stability falls within class $k$, and that the mixing layer thickness falls within class 1 . It should be recalled that $G(z)$ is a function of distance, stability, mixing layer thickness, and release height. The derivation of Equations (4.3.9) and (4.3.12) is discussed by Gifford (1968).

The Gaussian Plume based models embodied in Equations (4.3.1), (4.3.9), $(4.3 .10),(4.3 .11)$, and (4.3.12) estimate average concentrations for continuous releases with constant release rates. These concentrations may be used to evaluate dose rates. Before doses may be estimated, it is necessary to convert the average concentration to exposure. This is done by multiplying the average concentration by the time that the receptor is within the plume. In the case of a release of finite duration, the release duration may be used in computing the exposure.

\subsubsection{Diffusion Coefficients}

A large number of experiments have been conducted to evaluate atmospheric diffusion coefficients. The results of these experiments have been used to relate diffusion coefficients to atmospheric stability and distance from the source. The most familiar set of relationships are contained in the Pasquill-Gifford-Turner (PGT) curves (Gifford 1961 and 1976).

Martin and Tikvart (1968) and Tadmor and Gur (1969) present mathematical approximations, respectively, to the PGT vertical and horizontal diffusion coefficient curves. These parameterizations have the general form 


$$
\sigma=A x^{B}+C
$$

where $A, B$, and $C$ are assigned different values for computation of the horizontal and vertical diffusion coefficients. In computation of the horizontal diffusion coefficient, $A$ has been assigned the values for seven atmospheric stability classes, $B$ has a constant value of 0.9031 , and $C$ has a value of 0.0 . For computation of vertical diffusion coefficients, $A, B$, and $C$ are assigned values for seven stability classes and three distance ranges. The distance ranges are: 1) less than $100 \mathrm{~m}, 2$ ) 100 to $1000 \mathrm{~m}$, and 3) greater than $1000 \mathrm{~m}$. Values for $A, B$, and $C$ are given in Table 4.8. It should be noted that $A$ and $C$ are dimensional constants with units of $m^{1-B}$ and meters, respectively.

\subsubsection{Effective Release Height}

Computating ground-level concentrations downwind of elevated releases requires that the effective release height: be estimated. For free standing stacks, the effective release height is defined as

$$
h_{e}=h_{s}+h_{r}-h_{d}
$$

where $h_{\mathrm{S}}=$ the actual release height $(\mathrm{m})$

$h_{r}=$ the plume rise due to momentum and buoyancy (m)

$h_{d}=$ the plume downwash due to aerodynamic forces around the stack (m).

\begin{tabular}{|c|c|c|c|c|c|c|c|c|c|c|}
\hline \multirow{3}{*}{$\begin{array}{l}\text { Sta- } \\
\text { bility } \\
\text { Class } \\
\end{array}$} & \multirow[b]{3}{*}{ Ay } & \multicolumn{3}{|c|}{$A z$} & \multicolumn{3}{|c|}{$B z$} & \multicolumn{3}{|c|}{$\mathrm{Cz}$} \\
\hline & & & istance & ange & Dis & tance 1 & ange & & $\operatorname{tance}$ & Range \\
\hline & & 1 & 2 & 3 & 1 & 2 & 3 & & 2 & 3 \\
\hline A & 0.3658 & 0.192 & 0.00066 & 0.00024 & 0.936 & 1.941 & 2.094 & 0 & 9.27 & -9.6 \\
\hline B & 0.2751 & 0.156 & 0.0382 & 0.055 & 0.922 & 1.149 & 1.098 & 0 & 3.3 & 2 \\
\hline C & 0.2089 & 0.116 & 0.113 & 0.113 & 0.905 & 0.911 & 0.911 & 0 & 0 & 0 \\
\hline D & 0.1471 & 0.079 & 0.222 & 1.26 & 0.881 & 0.725 & 0.516 & 0 & -1.7 & -13 \\
\hline$E$ & 0.1046 & 0.063 & 0.211 & 6.73 & 0.871 & 0.678 & 0.305 & 0 & -1.3 & -34 \\
\hline$F$ & 0.0722 & 0.053 & 0.086 & 18.05 & 0.814 & 0.74 & 0.18 & 0 & -0.35 & -48.6 \\
\hline G & 0.0481 & 0.032 & 0.052 & 10.83 & 0.814 & 0.74 & 0.18 & 0 & -0.21 & -29.2 \\
\hline
\end{tabular}

TABLE 4.8. Constant Values for Diffusion Coefficient Evaluation 
Equation (4.3.14) may also be used to define the effective release height for stacks in the vicinity of buildings, provided that the stack height is sufficient such that plumes emitted avoid entering the building wakes. As a general rule, a stack 2.5 times the height of nearby buildings is assumed to be equivalent to a freestanding stack.

Briggs (1984) discusses both plume rise and downwash. Plume rise is directly related to the momentum and buoyancy of the effluent and is inversely related to the wind speed. In addition, the extent of plume rise is affected by atmospheric stability and may be limited by the top of the mixing layer. Evaluation of plume rise requires information on stack dimensions and flow, effluent density and temperature, and ambient air temperature. The following relationships from Briggs (1984) provide a consistent set of plume rise estimates for all wind and stability conditions.

Two parameters, buoyancy flux and momentum flux, are used in computing plume rise. The buoyancy flux parameter, $F_{b}$, is defined as

$$
F_{b}=g\left(\frac{\rho_{a}-\rho_{0}}{\rho_{a}}\right) w_{0} r_{0}^{2}
$$

where $g=$ the gravitational constant $\left(9.8 \mathrm{~m} / \mathrm{sec}^{2}\right)$

$$
\rho_{0}=\text { the density of the plume }\left(\mathrm{Kg} / \mathrm{m}^{3}\right)
$$

$\rho_{a}=$ the density of air $\left(\mathrm{Kg} / \mathrm{m}^{3}\right)$

$w_{0}=$ the stack exit velocity $(\mathrm{m} / \mathrm{sec})$

$r_{0}=$ the inside radius of the stack $(m)$.

The buoyancy flux parameter has dimensions of $\mathrm{m}^{4} / \mathrm{sec}^{3}$. The momentum flux parameter, $F_{m}$ is defined as

$$
F_{m}=\left(\rho_{0} / r_{a}\right) w_{0}^{2} r_{0}^{2}
$$

It has dimensions of $\mathrm{m}^{4} / \mathrm{sec}^{2}$.

The momentum component of plume rise, $h_{m}$, is assumed to be limited by atmospheric turbulence. It is given by 


$$
h_{m}=\frac{0.9\left[F_{m} /\left(U u^{\star}\right)\right]^{1 / 2}}{0.4+1.2\left(U / w_{0}\right)}
$$

where $U$ is the stack-height wind speed and $u *$ is a characteristic wind speed associated with turbulence. The relationship between the characteristic wind speed and the measured speed is discussed in Section 4.3.6, Wind Profiles.

The buoyancy component of plume rise, $h_{b}$, is estimated using three equations. During unstable and neutral atmospheric conditions, the buoyancy rise component is given by

$$
h_{b}=1.54\left[F_{b} /\left(U u^{*}\right)\right]^{2 / 3} h_{s}^{1 / 3}
$$

During stable atmospheric conditions with a release height wind speed of $1 \mathrm{~m} / \mathrm{sec}$ or more, the buoyancy rise component is given by

$$
h_{b}=2.6\left[F_{b} /\left(U_{s}\right]^{1 / 3}\right.
$$

where $s$ is a stability parameter. In stable atmospheric conditions, if the wind is less than $1 \mathrm{~m} / \mathrm{sec}$, the buoyant plume rise component is estimated as

$$
h_{b}=5.3\left[F_{b} / s^{3 / 2}\right]^{1 / 4}-6 r_{0}
$$

The stability parameter in Equations (4.3.19) and (4.3.20) is defined as

$$
s=\frac{g}{\theta} \frac{\partial \theta}{\partial z}
$$

where $g=$ the gravitational constant $(9.8 \mathrm{~m} / \mathrm{sec})$

$$
\begin{aligned}
\theta & =\text { the potential temperature of the air }\left({ }^{\circ} \mathrm{K}\right) \\
\partial \theta / \partial z & =\text { the potential temperature lapse rate })\left({ }^{\circ} \mathrm{K} / \mathrm{m}\right) .
\end{aligned}
$$

Near sea level, the stability parameter may be approximated as 


$$
s=c / T
$$

where $c$ is a constant that is defined by stability class, and $T$ is the ambient air temperature $\left({ }^{\circ} \mathrm{K}\right)$. The values of $c$ for $E, F$, and $G$ stability classes are $0.049,0.27$, and $0.49^{\circ} \mathrm{K} / \mathrm{sec}^{2}$, respectively.

When Equation (4.3.19) or $(4.3 .20)$ is used to compute the buoyant component of plume rise, the estimated rise is compared with the rise computed using Equation (4.3.18). The smaller of the plume rise estimates is then used in computing the final plume rise.

Final plume rise, which is the sum of the momentum and buoyant components, is subject to three constraints. If the release height is greater than the mixing layer thickness or if the stack exit velocity is equal to zero, final plume rise is set to zero. Otherwise, the rise is limited to one half of the distance between the top of the stack and the top of the mixing layer.

The plume rise component Equations (4.3.17), (4.3.18), (4.3.19), and (4.3.20), and the effective release height estimate in Equation (4.3.14) give the height of the plume after it has become essentially horizontal. These equations should not be used to estimate concentrations near the release point because small changes in effective release height result in large changes in estimated ground-level concentrations near the release point. If concentration estimates are needed for locations within a few hundred meters of a stack, the estimates should be made using more appropriate relationships. In most cases, concentration estimates near an elevated release should be viewed with caution, particularly if the release parameters or atmospheric conditions are not well known.

As the distance from the release point increases, the sensitivity of concentration as a function of release height decreases. In most atmospheric conditions, at distances greater than one or two kilometers, the concentration estimates for elevated release should not be sensitive to moderate changes in release height.

\subsubsection{Building-Wake Diffusion Model}

Atmospheric diffusion in building wakes is usually treated by modifying the Gaussian plume model to account for increased diffusion caused by 
buildings. However, analys is of building-wake diffusion data (Ramsdell 1988) shows that the usual methods of estimating the concentrations in wakes do not adequately describe the variations of the concentrations that are caused by changes in atmospheric conditions. An alternative model for centerline concentrations in building wakes, which was proposed by Ramsdell, is included in GENII. That model is based on a statistical analysis of diffusion data obtained at seven locations.

The basic form of the empirical building-wake diffusion model developed by Ramsdell (1988) is

$$
\bar{X} / Q^{\prime}=k x^{a} A^{b} U^{c} s^{d}
$$

where $\quad x=$ distance from the release point (m)

$A=$ projected building area $\left(\mathrm{In}^{2}\right)$

$S=$ atmospheric stability class; $1=A, 2=B, \ldots$

$k, a, b, c, d=$ parameters to be determined.

This model includes the same variables that have been included in previous models. However, it does not specify the way in which each of the variables enters the model. Multiple linear regression techniques were applied to building wake diffusion data for ground-level releases for a wide range of atmospheric conditions and distances to estimate parameter values for the model. Parameter values for the model are $k=150, a=-1.2, b=-1.2$, $c=0.68$, and $d=0.5$.

Although the basic wake model provides a "best-fit" to the data in a least squares sense, it is not expected to model the concentrations near the source unless the size of the opening through which the release occurs is small and the flow through the opening is negligible. Similarly, the model does not asymptotically approach the Gaussian plume model as the distance from the source becomes large. To correct these deficiencies, the basic model is incorporated in the following composite model:

$$
\bar{\chi} / Q^{\prime}=\frac{1}{\left(F_{0}+F_{p}+F_{w}\right)}
$$


where $F_{0}=$ the flow rate associated with the release

$F_{p}=$ the denominator of the Gaussian plume model defined in

Equation (4.3.1) multiplied by a factor of 2

$F_{W}=$ the reciprocal of the wake model defined in Equation (4.3.23).

In this model, the initial flow, $F_{0}$, is constant and does not contribute significantly at large distances. Both $F_{p}$ and $F_{w}$ increase as $x$ increases. To get the model to asymptotically approach the Gaussian plume model at large distances, the wake-induced diffusion is limited by placing an upper limit on the distance used in the wake model. Further, the limit should be related to building size. In GENII, a characteristic building dimension equal to the square root of the building area is used to limit the wake diffusion contribution in Equation (4.3.24). $F_{W}$ is allowed to increase until the distance increases to 20 times the characteristic building dimension. At larger distances, the wake diffusion contribution to Equation (4.3.24) is held constant at its maximum value.

The conservative approach to estimating the concentrations downwind of short stacks and roof-top vents is to assume that a release takes place at ground level unless the release point is 2.5 times the building height. However, a significant body of literature exists to support a less conservative assumption in which the behavior of plumes released from short stacks and roof-top vents depends on the ratio between the vertical velocity of the effluent and the wind speed at release height. When the ratio is large, plumes escape the wake; when it is small, they remain in the wake; and when the ratio has an intermediate value, they escape the wake part of the time and are entrained in the wake the remainder of the time.

Following the wake diffusion experiments at the Millstone Nuclear Power Station, Johnson et al. (1975) suggested a model, which they called a Split-H model, to account for this behavior of elevated releases. A modified version of the Split-H model is included in the U.S. Nuclear Regulatory Commission (NRC) Regulatory Guide 1.111 (NRC 1977c) and is implemented in the XOQDOQ model (Sagendorf, Goll, and Sandusky 1982), which is used in evaluating the consequences of routine releases from nuclear power plants. The NRC implementation of the Split-H model is included in the GENII building-wake model. 
The Split-H model is applied to releases in which the actual release height is equal to or greater than the height of the tallest building in the area. Assuming this condition, a release is considered to be elevated when the ratio of vertical velocity to wind speed is equal to or greater than 5. If this ratio is between 1 and 5 , the concentration is computed assuming that a portion of the release is elevated and the remainder is at ground level. otherwise, the ratio is less than 1 , and the release is considered to be a ground-level release.

The exact form of the NRC implementation of the model is

$$
\bar{\chi} / Q^{\prime}=M\left(\bar{\chi} / Q^{\prime}\right)_{\text {entr }}+(1-M)\left(\bar{\chi} / Q^{\prime}\right)_{\text {elev }}
$$

where

$$
\begin{aligned}
\bar{X} / Q^{\prime}= & \text { the normalized concentration predicted by the Split-H } \\
& \text { model } \\
M= & \text { the fraction of time that the plume is entrained in the } \\
& \text { building wake } \\
\left(\bar{X} / Q^{\prime}\right) \text { entr }= & \text { the normalized concent:ration in the building wake } \\
& \text { predicted for a ground-level release } \\
\left(\bar{X} / Q^{\prime}\right) \text { elev }= & \text { the normalized concentration at ground level predicted } \\
& \text { for an elevated release. }
\end{aligned}
$$

5 The fraction of the time that the plume is entrained in the wake is estimated from the ratio of the effluent vertical velocity $\left(w_{0}\right)$ to the release height wind speed $\left(U_{r}\right)$ according to:

$$
M= \begin{cases}1 & w_{0} / U_{r}<1.0 \\ 2.58-1.58\left(w_{0} / U_{r}\right) & 1.0<=w_{0} / U_{r}<1.5 \\ 0.3-0.06\left(w_{0} / U_{r}\right) & 1.5<=w_{0} / U_{r}<5.0 \\ 0 & w_{0} / U_{r}>=5.0 .\end{cases}
$$

The 10-m wind speed is used in the GENII wake model except when the Split-H procedure is used. Then the release-height wind will be used to determine the fraction of time that the release is in the wake and will be used to estimate diffusion when the plume escapes the wake. 


\subsubsection{Deposition and Depletion}

To estimate doses to individuals from contaminated soil and the farm product ingestion pathway, it is necessary to first estimate the amount of each radionuclide deposited on the ground. Two deposition mechanisms are frequently included in atmospheric models. The first mechanism, dry deposition, involves the settling of material on a surface as a result of gravitation and turbulent transport. In the second mechanism, called wet deposition, precipitation such as rain forms with particulate material, collides with particles, or absorbs gases and then carries the material to the surface. Wet deposition occurs during precipitation and results in a higher rate of surface contamination than dry deposition. However, dry deposition occurs whenever the concentration of material near the surface is greater than zero. As a result, in the vicinity of a source of routine release of radioactive nuclides, dry deposition is likely to be the primary process by which contamination occurs. Because of the infrequent precipitation in the Hanford environment, wet deposition is not considered further.

Although the deposition rate may be of some interest, the dose rate due to deposited material is a function of the total deposited material. The total surface contamination is given by

$$
c_{s}=\bar{\chi} v_{d} t
$$

where $C_{s}=$ the surface contamination $\left(\mathrm{Ci} / \mathrm{m}^{2}\right)$

$v_{\mathrm{d}}=$ the deposition velocity $(\mathrm{m} / \mathrm{sec})$

$\bar{\chi}=$ the average air concentration $\left(\mathrm{Ci} / \mathrm{m}^{3}\right)$

$t=$ the time period for which the average concentration was computed (sec).

The deposition velocity is a constant of proportionality between surface contamination rate and the concentration of contaminants in air. Deposition velocity depends on the substance and the surface, and the values of deposition velocity cover a wide range. Sehmel $(1980,1984)$ summarizes reported deposition velocities for many substances including iodine. For noble gases, a deposition velocity of zero is used; for most particulates, a deposition 
velocity of $0.001 \mathrm{~m} / \mathrm{sec}$ is used; and for iodines, a deposition velocity of $0.01 \mathrm{~m} / \mathrm{sec}$ is used.

Deposition on surfaces results in depletion of material from the plume. Although several methods exist to account for this depletion, they are generally not incorporated in simple atmospheric models even when deposition is included. As a result, air concentration estimated by simple models are biased to the high side.

\subsubsection{Wind Profiles}

The algorithms within the GENII code are based on wind speeds at specific heights. The height at which meteorological data have been obtained may or may not correspond to heights appropriate for the use in GENII. Consequently, a set of algorithms is provided within GENII to adjust wind speeds from the height of measurement to the height required. The algorithms are based on the diabatic wind profile model described by Panofsky and Dutton (1984). In this model, wind speed is a function of height above ground, surface roughness, the characteristic turbulence velocity, and a characteristic length associated with the atmospheric boundary layer.

The general form of the wind profile model is

$$
U(z)=\frac{u^{*}}{0.4} \ln \left(\frac{z}{z_{0}}-\Psi\right)
$$

where $U(z)=$ the wind speed at height $z(\mathrm{~m} / \mathrm{sec})$

$u^{*}=$ the characteristic velocity $(\mathrm{m} / \mathrm{sec})$

$z=$ the height above ground ( $m$ )

$z_{0}=$ the surface roughness length $(\mathrm{cm})$

$\Psi=$ a dimensionless empirical function of the ratio between the height above ground and the characteristic length, L, associated with the atmospheric boundary layer called the MoninObukov length (Golder 1972).

The Monin-Obukov length is positive for stable atmospheric conditions, negative for unstable conditions, and infinite for neutral conditions. Estimates of $L^{-1}$ used in GENII are based on Golder (1972) which relates the 
inverse of the Monin-Obukov length to stability class and surface roughness length.

Wind profile data have been used to evaluate $\Psi$, which is a function of stability. For neutral stability, $\Psi$ is equal to zero, and Equation (4.3.28) simplifies to the familiar logarithmic profile. For stable atmospheric conditions, is defined by

$$
\Psi=-5 \mathrm{z} / \mathrm{L}
$$

where $L$ is the Monin-Obukov length in meters. The definition of $\Psi$ for unstable conditions is considerably more complex. It is

$$
\Psi=\ln \left[\left(0.5+\frac{\phi^{2}}{2}\right)\left(0.5+\frac{\phi}{2}\right)^{2}-2 \operatorname{Atan} \phi+\frac{1}{2}\right]
$$

where $\phi$ is defined as

$$
\phi=(1-16 z / L)^{1 / 4}
$$

\subsubsection{Atmospheric Dispersion Model Limitations}

The atmospheric dispersion models discussed are all simple models. They yield reasonable yet conservative concentration estimates, provided that they are not misapplied.

As a general rule, the use of Equations (4.3.1) and (4.3.9) should be limited to potential releases of. 30 minutes to 4 hours in duration. Equations (4.3.10) and (4.3.11) can be used to assess the consequences of potential releases 2 to 8 hours in duration, and Equation (4.3.12) can be used for potential releases with duration of 4 hours and longer. The fact that these ranges overlap should not imply that the models will yield identical results for release durations that fall within the overlap; they won't. When a release duration falls within an overlap, the choice between alternative equations should be based on an evaluation of the physical situation being modeled, and this evaluation should consider the source characteristics and meteorological situation. 
The derivation of the dispersion models explicitly includes the assumptions that the release is from a point source and that the effluent travels in a straight line once it is released. Considering the point source assumption, Equations (4.3.1) and (4.3.9) through (4.3.11) should not be used to estimate concentrations too near the source. For relatively small sources, the models may be applied to estimate concentrations as close to the source as $800 \mathrm{~m}$. However, if the source is in or near large buildings, these models should not be used to estimate concentrations closer to the source than $1600 \mathrm{~m}$. Considering the straight-line assumption, the equations should not be used to estimate concentrations further than $80 \mathrm{~km}$ from the source under ideal conditions of flat terrain and no systematic spatial variations of the wind field. If topographic features may alter the wind field, the models should not be used to estimate concentrations on the opposite side of the features from the source.

The release duration and travel time to the receptor should also be considered when determining a maximum range for estimating concentrations. If the release is short compared to the travel time, assuming that the effluent travels at the wind speed, the equations may not provide realistic estimates of the concentration. Similarly, if the time required for the effluent to travel from the source to the receptor is more than a few hours, atmospheric conditions will likely change sufficiently during the transit period to make the concentration estimates unrealistic. Specifically, concentrations from releases made near suririse will be systematically overestimated, and concentrations for releases near sunset will be underestimated. These considerations are particularly important when using Equations $(4.3 .1),(4.3 .9),(4.3 .10)$, and $(4.3 .11)$.

Other meteorological conditions of concern in applying Equations (4.3.1), (4.3.9), (4.3.10), and (4.3.11) include drainage winds, passages of fronts and other storms, and periods of low wind speeds. Each of these conditions can result in a significant departure from the modeling assumption that the release takes place in meteorological conditions that are not a function of time. Low wind speeds pose a particular problem because

1) the wind direction becomes poorly defined as the speed decreases and 2) the equations become undefined mathematically. 


\subsection{AIR SUBMERSION}

Persons submersed in a plume of airborne material will receive radiation exposure from gamma and $x$-rays emitted external to the body. This exposure is generally referred to as external dose from air submersion and is dependent upon the type of radiation, the energy of the radiation, and the spatial distribution of the airborne radionuclides surrounding the receptor. Two methods are available in GENII to estimate the dose: 1) the semi-infinite plume model and 2) the finite plume model.

The semi-infinite plume model is based on the assumption that the plume is semi-infinite in size (bounded by the ground plane) and that the energy deposition per unit volume of air is equal to the rate of energy emission per unit volume. In contrast, the finite plume model considers the size and shape of the plume to estimate the actual dose rate at the center of the plume at ground level. Both methods may be applied to acute and chronic release situations.

\subsubsection{Semi-Infinite Plume Model}

For acute releases of radionuclides, the semi-infinite plume model for the air submersion dose is represented as follows:

$$
D 1_{i}=Q_{i}(E / Q) D F_{s i}
$$

where $\quad D 1_{i}=$ individual dose from external exposure to the plume for radionuclide $i$ (rem)

$$
\begin{aligned}
Q_{i}= & \text { total activity of radionuclide } i \text { released during the acute } \\
& \text { release period (curies) } \\
E / Q= & \text { acute release atmospheric dispersion factor at the exposure } \\
& \text { location }\left(\mathrm{sec} / \mathrm{m}^{3}\right) \\
D F_{s i}= & \text { submersion dose factor precalculated from EXTDF program } \\
& \left(\text { rem per } C i-\mathrm{sec} / \mathrm{m}^{3}\right) .
\end{aligned}
$$

For chronic releases, the air submersion dose is calculated similarly:

$$
D I_{i}=Q_{i}^{\prime}\left(\bar{\chi} / Q^{\prime}\right) D F_{s i}
$$


where $Q_{i}^{\prime}$ is the annual average release rate of radionuclide $i$ ( $C i /$ year), $\bar{\chi} / Q^{\prime}$ is chronic release atmospheric dispersion factor at the exposure location $\left(\mathrm{sec} / \mathrm{m}^{3}\right)$, and other terms are as defined above for acute releases.

\subsubsection{Finite Plume Model}

When the finite plume model is used, the geometry must be considered in evaluating the exposure. The evaluation involves use of pre-calculated factors representing volume integrals over the plume dimensions. Parameters important to this evaluation are plume size, release height, wind speed, and photon energy. Two empirical models are available, one for acute releases based on the bivariate Gaussian plume mode1, and one for chronic releases based on the crosswind averaged (sector width) Gaussian dispersion model. The exposure may be represented as follows for acute releases:

$$
D 1_{i} \equiv Q_{i} \sum_{j} D R A_{j} E_{i j} F c_{j}
$$

where $\mathrm{DRA}_{j}=$ acute release finite plume dose rate factor for photons in energy group $\mathrm{j}$ at the exposure location ( $\mathrm{rad} \mathrm{dis)/(MeV} \mathrm{Ci)}$

$E_{i j}=$ effective photon energy released by radionuclide $i$ in energy group $\mathrm{j}$ (MeV/dis)

$F c_{j}=$ surface-dose-to-effective-dose equivalent conversion factor for photon energy group $j$ (rem/rad).

The surface-dose-to-effective-dose-equivalent conversion factor converts from air dose to effective dose equivalent for photons in energy group $j$. The photon energy emission data are read from a data library which contains photon data grouped into the six energy groups indicated in Table 4.9. The table also gives the surface-dose-to-effective-dose-equivalent conversion factors for each energy group. Photon data for each radionuclide are contained in the data library. 
TABLE 4.9. Photon Energy Group Definitions and Conversion Factors Used in Finite-Plume Air Submersion Calculations

\begin{tabular}{ccccc} 
Group & $\begin{array}{c}\text { Photon Energy } \\
\text { Range, MeV }\end{array}$ & $\begin{array}{c}\text { Average Photon } \\
\text { Energy, MeV }\end{array}$ & $\begin{array}{c}\text { Surface Do } \\
\text { EDE Factor, }\end{array}$ \\
\cline { 2 - 2 } 1 & $<0.3$ & & 0.15 & 0.67 \\
2 & $0.3-0.5$ & & 0.40 & 0.66 \\
3 & $0.5-1.0$ & 0.75 & 0.67 \\
4 & $1.0-1.5$ & 1.25 & 0.71 \\
5 & $1.5-2.0$ & 1.75 & 0.75 \\
6 & $>2.0$ & 2.25 & 0.78
\end{tabular}

For chronic releases, the air submersion dose is evaluated as follows:

$$
D 1_{i}=Q^{\prime} \sum_{j} D R C_{j} E_{i j} F C_{j}
$$

where $D R C_{j}$ is the chronic release finite plume dose rate factor for photons in energy group $\mathrm{j}$ at the exposure location ( $\mathrm{rad} \mathrm{dis}) /(\mathrm{MeV} \mathrm{Ci})$.

The acute release model uses an empirical function to evaluate the finite plume dose factor $\left(D R A_{j}\right)$, depending on plume size $\left(\sigma_{y}\right.$ and $\left.\sigma_{z}\right)$, plume release height $\left(h_{e}\right)$, and photon energy (subscript $j$ ). The empirical function was developed from finite plume dose rate factors calculated using the computer program SUBDOSA (Strenge, Watson, and Houston 1975). The analysis used the curve fitting program SSQMIN (Brown and Lin 1973; Brown 1973) to evaluate the constants of the empirical function. For ground-level releases, the dose rate factor is evaluated as follows:

$$
\mathrm{DRAo}_{j}=\mathrm{K}_{\mathrm{j}} \mathrm{Ro}_{\mathrm{j}} /\left(2 \pi \sigma_{\mathrm{y}} \sigma_{\mathrm{z}} \mathrm{u}\right)
$$

where $\quad K_{j}=$ units conversion factor ( $\left.\mathrm{rad} \mathrm{m^{3 }} \mathrm{dis}\right) /(\mathrm{MeV} \sec \mathrm{C} i$ )

$\mathrm{Ro}_{j}=$ ground-level release finite-plume correction factor for energy group $j$ (dimensionless)

$\sigma_{y}=$ crosswind horizontal standard deviation of plume size at the downwind exposure location (m)

$\sigma_{z}=$ crosswind vertical standard deviation of plume size at the 
downwind exposure location ( $\mathrm{ml}$

$u=$ average wind speed $(\mathrm{m} / \mathrm{sec})$.

The factor $R_{j}$ is evaluated empirically as follows:

$$
\begin{gathered}
R_{j}=a_{1 j}\left(\sigma_{g}\right) b_{1 j} \text { for } \sigma_{g} \leq 100 \text { meters } \\
R o_{j}=a_{2 j}\left(\sigma_{g}\right) b_{2 j} \text { for } 100<\sigma_{g}<600 \text { meters } \\
R o_{j} \leq 1 \text { for all } \sigma_{g}
\end{gathered}
$$

where $\quad \sigma_{g}=$ geometric mean of plume dimensions (m)

$$
\begin{aligned}
\sigma_{g} & =\left(\sigma_{y} \sigma_{z}\right)^{1 / 2} \\
a_{1 j}, a_{2 j}, b_{1 j}, b_{2 j} & =\text { empirical constants for energy group } j .
\end{aligned}
$$

Values for the empirical coefficients are given in Table 4.10.

For elevated releases, the dose rate factor is evaluated using the ground-level correction factor plus an effective release height as follows:

$$
\mathrm{DRA}_{j}=\mathrm{DRAo}_{j} \exp \left[-\left(H_{j}^{*}\right)^{2} /\left(2 \sigma_{z}\right)\right]
$$

\begin{tabular}{|c|c|c|c|c|}
\hline $\begin{array}{l}\text { Energy } \\
\text { Group } \\
\end{array}$ & $a_{1 j}$ & $\mathrm{a} 2 \mathrm{j}$ & $b_{1 j}$ & $b_{2 j}$ \\
\hline 1 & 0.007062 & 0.9623 & 0.1651 & 0.2779 \\
\hline 2 & 0.007915 & 0.9222 & 0.1514 & 0.2813 \\
\hline 3 & 0.008279 & 0.8839 & 0.08769 & 0.3713 \\
\hline 4 & 0.007696 & 0.8854 & 0.07532 & 0.3900 \\
\hline 5 & 0.006964 & 0.8941 & 0.06136 & 0.4215 \\
\hline 6 & 0.005852 & $0: 9038$ & 0.04238 & 0.4740 \\
\hline
\end{tabular}

$$
\text { where } \begin{aligned}
H_{j}^{*} & =\text { effective plume height for energy group } j(m) \\
H_{j}^{*} & =H_{j} h\left(\sigma_{y} / \sigma_{z}\right) P_{j}
\end{aligned}
$$

where $h=$ effective release height $(m)$

TABLE 4.10 Empirical Constants for the Ground-Level Correction Factor, Roj 


$$
\begin{aligned}
H O_{j} & = \begin{cases}1-d_{j}\left(h / \sigma_{z}\right) e_{j} & \text { for } h / \sigma_{z} \leq 10 \\
f_{j} /\left(h / \sigma_{z}\right) & \text { for } h / \sigma_{z}>10\end{cases} \\
P_{j} & =c_{j} \exp \left(-0.015 \sigma_{z}\right)
\end{aligned}
$$

where $c_{j}, d_{j}, e_{j}, f_{j}$ are empirical constants for energy group $j$. Values for the empirical constants are given in Table 4.11.

\begin{tabular}{|c|c|c|c|c|}
\hline $\begin{array}{l}\text { Energy } \\
\text { Group } \\
\end{array}$ & $c_{j}$ & ${ }^{d} j$ & $e_{j}$ & $\mathbf{f}_{j}$ \\
\hline 1 & -0.19644 & 0.31637 & 0.38578 & 2.8173 \\
\hline 2 & -0.18854 & 0.29041 & 0.41921 & 2.8136 \\
\hline 3 & -0.18610 & 0.28409 & 0.42522 & 2.8053 \\
\hline 4 & -0.18601 & 0.28408 & 0.42532 & 2.8049 \\
\hline 5 & -0.18660 & 0.28414 & 0.42572 & 2.8039 \\
\hline 6 & -0.18710 & 0.28418 & 0.42610 & 2.8031 \\
\hline
\end{tabular}

TABLE 4.11. Empirical Constants for Effective Release Height, $\mathrm{HJ}_{\mathrm{J}}^{*}$

A similar empirical model is used to evaluate the finite plume dose rate factors for chronic releases $\left(D_{R} C_{j}\right)$. However, the release height dependence is handled differently, in that the empirical representation is defined for specific release height values. Preliminary evaluation of dose rate factors is made for the specific release heights, and these factors are then interpolated to provide dose rate factors for the desired release height. The empirical function development for chronic release dose rate factors is based on calculations performed using the KRONIC subprogram FDOSE (Strenge and watson 1973) which performs the dose rate integration over the sectoraveraged plume volume.

For ground-level releases, the dose rate factors are evaluated as follows for values of $\sigma_{\mathrm{Z}} \leq 200$ meters:

$$
\mathrm{DRCO}_{j}=\mathrm{K}_{\mathrm{j}}\left[\mathrm{A}_{\mathrm{j}}+\mathrm{x}_{3} \ln \sigma_{\mathrm{z}}\right]
$$


where $A_{j}= \begin{cases}x_{2} & \text { when } x>901 \text { meters } \\ x_{4}+x_{1} \ln x & \text { when } x \leq 901 \text { meters }\end{cases}$

$$
\left[A_{j}+\ln \sigma_{z}\right] \leq 1.0
$$

and as follows for values of $\sigma_{z}>200$ meters:

$$
\mathrm{DRCo}_{j}=x_{5} /\left(\sigma_{z} W\right)
$$

where

$$
\begin{aligned}
\mathrm{DRCO}_{\mathrm{j}}= & \text { ground-level dose rate factor for photons in energy } \\
& \text { group } \mathrm{j} \text { (rem dis } / /(\mathrm{MeV} \mathrm{C}) \\
\mathrm{x}= & \text { downwind distance (meters) } \\
\mathrm{W}= & \text { sector width at clownwind distance } \mathrm{x} \text { (meters) }
\end{aligned}
$$

$\mathrm{K}_{j}, x_{1}, x_{2}, x_{3}, x_{4}, x_{5}=$ empirical constarits.

Value $s$ for the empirical constants are given in Table 4.12.

The user must perform the following steps in evaluating chronic dose rate factors for elevated releases:

1. If $\sigma z<S 1_{h}$, set $\sigma_{z}=S 1_{h}$.

2. If $\sigma_{z}<S 2_{h}$, set $x x=$ maximum of $x$ and 2000 meters.

and if $\sigma_{z}<S 3_{h}, E F=x 1_{h j}+x 2_{h j} \ln (x x)$.

or if $\sigma_{z} \geq S 3_{h}, E F=E F\left(X 4_{h j}+x 5_{h j} x x\right)$

Using these values,

$$
\mathrm{DRC}_{h j}=0.398942 \mathrm{FK}_{\mathrm{j}} \sigma_{\mathrm{z}} \times 3 \mathrm{hj} \mathrm{EF/(W \sigma _{z } )}
$$

This completes the evaluation when $\sigma_{\mathrm{z}}<\mathrm{S}_{\mathrm{h}}$.

3. If $\sigma_{z} \geq S 2_{h}$ and $\sigma_{z}<S 4_{h}$ '

and if $x>D x_{h j}$ '

evaluate $E G=\exp \left[X \sigma_{h j}+X_{h j}\right.$ In $\left.\sigma_{z}\right]$ if $x>D x_{h j}$.

Otherwise, $E G=X 8_{h j}\left(x{ }_{h j}\right) \exp \left(X \sigma_{h j}+X 7_{h j} \ln \sigma_{z}\right)$

and then evaluate

$$
\mathrm{DRC}_{j h}=0.398942 \mathrm{FK}_{\mathrm{j}} \sigma_{\mathrm{z}} \mathrm{EG} /\left(\mathrm{W} \sigma_{\mathrm{z}}\right)
$$

This completes the evaluation when $S 2_{h} \leq \sigma_{z}<S 4_{h}$.

4. If $\sigma_{z} \geq S 4_{h}$,

$$
\mathrm{DRC}_{h j}=A_{h j} /\left(W \sigma_{z}\right)
$$


TABLE 4.12. Empirical Constants for Ground-Level Sector Dose Rate Factors

\begin{tabular}{|c|c|c|c|c|c|c|}
\hline $\begin{array}{l}\text { Energy } \\
\text { Group }\end{array}$ & $A_{k}$ & $x_{1}$ & $\times 2$ & $\times 3$ & $x_{4}$ & $\times 5$ \\
\hline 1 & 0.0040 & 0.2154 & 1.232 & -0.1732 & -0.1374 & 0.191 \\
\hline 2 & 0.0038 & 0.2056 & 1.328 & -0.2005 & 0.0005606 & 0.199 \\
\hline 3 & 0.0038 & 0.1763 & 1.246 & -0.1961 & 0.1066 & 0.182 \\
\hline 4 & 0.0036 & 0.1763 & 1.246 & -0.1961 & 0.1066 & 0.182 \\
\hline 5 & 0.00342 & 0.1745 & 1.207 & -0.1888 & 0.07537 & 0.182 \\
\hline 6 & 0.00342 & 0.1500 & 1.0385 & -0.1609 & 0.05711 & 0.182 \\
\hline
\end{tabular}

Parameters in the above calculational steps are previously defined in addition to the empirical constants $A_{h j}, D x h j, S 1_{h}$ through $S 4 h$, and $X 1_{h j}$ through $X 9_{\mathrm{hj}}$. Values for the "S" constants (dependent only on release height) are given in Table 4.13. Values for $A_{h j}, D \times h j$, and $X 1_{h j}$ through $X 9_{h j}$ are given in Tables 4.14 through 4.24 , respectively.

\section{TABLE 4.13. Values for "S" Parameters}

\begin{tabular}{|c|c|c|c|c|c|}
\hline$\frac{\text { Release }}{\text { Meters }}$ & $\frac{\text { leight }}{\text { Index }}$ & $\sin$ & $\mathrm{S} 2 \mathrm{~h}$ & $S 3 h$ & $S 4 h$ \\
\hline 50 & 1 & 5 & 80 & 20 & 400 \\
\hline 100 & 2 & 15 & 100 & 30 & 300 \\
\hline 150 & 3 & 30 & 150 & 30 & 400 \\
\hline 200 & 4 & 53 & 150 & 40 & 500 \\
\hline 250 & 5 & 60 & 150 & 40 & 500 \\
\hline
\end{tabular}


IABLE 4.14. Parameter Ahj Values

Release Height Meters Index

\begin{tabular}{|c|c|c|c|c|c|c|c|}
\hline \multirow{2}{*}{\multicolumn{2}{|c|}{$\begin{array}{l}\text { Release Height } \\
\text { leters Index }\end{array}$}} & \multicolumn{6}{|c|}{ Energy Group Index } \\
\hline & & 1 & 2 & 3 & 4 & 5 & 6 \\
\hline 50 & 1 & 0.1909 & 0.1995 & 0.1930 & 0.1897 & 0.1888 & 0.1916 \\
\hline 100 & 2 & 0.1904 & 0.1947 & 0.1947 & 0.1908 & 0.1908 & 0.1927 \\
\hline 150 & 3 & 0.1909 & 0.1974 & 0.1905 & 0.1875 & 0.1897 & 0.1914 \\
\hline 200 & 4 & 0.1900 & 0.1971 & 0.1902 & 0.1873 & 0.1893 & 0.1914 \\
\hline 250 & 5 & 0.1897 & 0.1968 & 0.1899 & 0.1870 & 0.1890 & 0.1911 \\
\hline
\end{tabular}

TABLE 4.15. Parameter Dxhj Values

Release Height Meters Index

$\begin{array}{rr}50 & 1 \\ 100 & 2 \\ 150 & 3 \\ 200 & 4 \\ 250 & 5\end{array}$

\begin{tabular}{rrrrrr}
\hline 1 & 2 & $-\frac{3}{3}$ & $\frac{4}{1200}$ & $\frac{5}{1150}$ & $\frac{6}{1500}$ \\
900 & 1000 & 950 & 1200 & 0 & 0 \\
0 & 0 & 0 & 0 & 0 & 0 \\
250 & 0 & 0 & 0 & 0 & 0 \\
0 & 0 & 0 & 0 & 0 & 0 \\
0 & 0 & 0 & 0 & 0 & 0
\end{tabular}

TABLE 4.16. Parameter $X 1_{h j}$ Values

\begin{tabular}{|c|c|c|c|c|c|c|c|}
\hline \multicolumn{2}{|c|}{ Release Height } & \multicolumn{6}{|c|}{ Energy Group Index } \\
\hline Meters & Index & 1 & 2 & 3 & 4 & 5 & 6 \\
\hline 50 & 1 & -0.0079836 & -0.0068464 & -0.0052439 & -0.0051147 & -0.0048566 & -0.0043104 \\
\hline 100 & 2 & -0.002566 & -0.002611 & $-0.00261 ?$ & -0.002612 & -0.0025281 & -0.001543 \\
\hline 150 & 3 & -0.0003225 & -0.0004380 & -0.00037150 & -0.0003687 & -0.0003970 & -0.0004192 \\
\hline 200 & 4 & -0.0000072393 & -0.0000218405 & -0.00002137361 & -0.0000315728 & -0.000055851 & -0.000055851 \\
\hline 250 & 5 & $-2.78157 \mathrm{E}-7$ & $-2.1792 E-6$ & $-5.795351:-6$ & $-7.382326 E-6$ & $-1.12916 E-5$ & $-1.99375 E-5$ \\
\hline
\end{tabular}


TABLE 4.17. Parameter $X 2 \mathrm{hj}$ Values

Release Height

\begin{tabular}{rrr}
\hline 50 & & 1 \\
100 & 2 \\
150 & 3 \\
200 & 4 \\
250 & 5
\end{tabular}

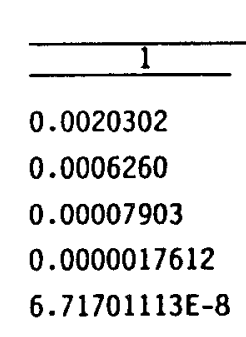

$\frac{2}{0.0017336}$
0.0006320
0.0001071
0.000005309
$5.25722 \mathrm{E}-7$

$\frac{1}{3}$
0.0013365
0.0006176
0.000091789
0.0000069799
$1.39556 \mathrm{E}-6$

$\frac{4}{4}$
0.0012986
0.0006642
0.0000901
0.0000076741
$1.78079 \mathrm{E}-6$

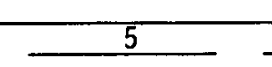

0.0012306

0.00060655

0.00009696

0.0000135817

$2.72453 \mathrm{E}-6$

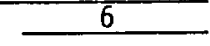

0.0010886

0.0003799

0.00010234

0.0000135817

4.815094E-6

TABLE 4.18. Parameter $\times 3 \mathrm{hj}$ Values

Release Height Meters Index

50

100

150

200

250

1
2
3
4
5

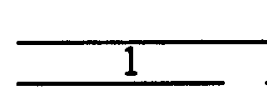

Energy Group Index

1.10853

1.12874

1.2196

1.200

1.51233

1.43132

2.193499

1.95538

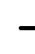
3

1.16066

4

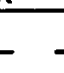

5

1.200

1.15024

1.14787

1.200

1.1124

1.14342

1.42964

1.41711

1.3893

1.167

2.76218724

2.337204

1.86565

1.832157

1.6790251 .679025

2.109784

2.045505

1.950043

1.811961

TABLE 4.19. Parameter $X 4 h j$ Values

Release Height Meters Index

\begin{tabular}{|c|c|c|c|c|c|}
\hline \multicolumn{6}{|c|}{ Energy Group Index } \\
\hline 1 & 2 & $\frac{3}{3}$ & 4 & 5 & 6 \\
\hline 1.06496 & 1.05508 & 1.03420 & 1.0384 & 1.03637 & 1.036195 \\
\hline 1.243 & 1.244 & 1.244 & 1.244 & 1.3268 & 1.3207 \\
\hline 1.36939 & 1.37817 & 1.3972 & 1.3934 & 1.38817 & 1.37466 \\
\hline 1.48126 & 1.45935 & 1.48287 & 1.457285 & 1.416737 & 1.416737 \\
\hline 1.50715482 & 1.462071 & 1.4591397 & 1.430768 & 1.410792 & 1.376726 \\
\hline
\end{tabular}


TABLE 4.20. Parameter $\times 5 h j$ Values

\begin{tabular}{|c|c|}
\hline$\frac{\text { Release }}{\text { Meters }}$ & $\frac{\text { Height }}{\text { Index }}$ \\
\hline 50 & 1 \\
\hline 100 & 2 \\
\hline 150 & 3 \\
\hline 200 & 4 \\
\hline 250 & 5 \\
\hline
\end{tabular}

\begin{tabular}{|c|c|c|c|c|c|}
\hline \multicolumn{6}{|c|}{ Energy Group Index } \\
\hline 1 & 2 & 3 & 4 & 5 & 6 \\
\hline-0.00014497 & -0.000120429 & -0.000107396 & -0.000102495 & -0.000089844 & -0.0000729814 \\
\hline-0.0001404 & -0.00009759 & -0.00009817 & $-1 . E-8$ & -0.0000285 & -0.00008758 \\
\hline-0.000060141 & 0.000016914 & 0.00006756 & 0.000113459 & 0.00015535 & 0.00023030 \\
\hline-0.0000100728 & 0.000094251 & 0.000178964 & 0.000241717 & 0.000401386 & 0.000401386 \\
\hline $2.31697304 E-5$ & $1.41925 \mathrm{E}-4$ & $2.52501 E-4$ & $3.34745 E-4$ & $4.020886 \mathrm{E}-4$ & $5.244587 E-4$ \\
\hline
\end{tabular}

IABLE 4.21. Parameter $\times 6 h j$ Values

Release Height Meters Index

$50 \quad 1$

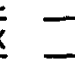

$100 \quad 2$

$$
-2.2101
$$

$\frac{2}{2}-\frac{3}{3}$

1503

$-2.51$

$-2.2101$

$$
-2.2827
$$

$-2.51$

$-2.622$

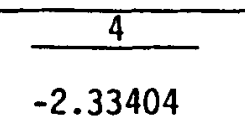

$\begin{array}{ll}\frac{5}{-2.411} & \frac{6}{-2.411} \\ -2.622 & -2.6173\end{array}$

$200 \quad 4$

$-2.83872$

$-2.83001$

$-2.86357$

$-2.622$

$-2.622$

$-2.6173$

250

$$
-3.20158
$$

$-3.19662$

$-3.17113$

$-2.87222$

$-2.88118$

$-2.90774$

$-4.11710167$

$-4.002866$

$-3.882893$

$-3.12888$

$-2.819186 \quad-2.819186$

$-3.809661$

\begin{tabular}{|c|c|c|c|c|c|c|c|}
\hline Release & Height & & & Eriergy Gr & up Index & & \\
\hline Meters & Index & 1 & 2 & -3 & 4 & 5 & 6 \\
\hline 50 & 1 & -0.6205 & -0.6205 & $-C 1.6205$ & -0.6205 & -0.6205 & -0.6205 \\
\hline 100 & 2 & -0.568 & -0.568 & -0.568 & -0.568 & -0.568 & -0.5843 \\
\hline 150 & 3 & -0.53759 & -0.53616 & -0.54130 & -0.54504 & -0.54449 & -0.54523 \\
\hline 200 & 4 & -0.49928 & -0.49664 & -0.51172 & -0.52408 & -0.58410 & -0.58410 \\
\hline 250 & 5 & -0.3576202 & -0.37317 & -0.40363 & -0.42112 & -0.44262 & -0.49152 \\
\hline
\end{tabular}

$-3.688744$

$-3.434422$

TABLE 4.22. Parameter $X 7_{\text {hj }}$ Values 
TABLE 4.23. Parameter $X 8_{\mathrm{hj}}$ Values

\begin{tabular}{|c|c|c|c|c|c|c|c|}
\hline \multicolumn{2}{|c|}{ Release Height } & \multicolumn{6}{|c|}{ Energy Group Index } \\
\hline Meters & Index & 1 & 2 & 3 & 4 & 5 & 6 \\
\hline 50 & 1 & 0.1525 & 0.10602 & 0.10084 & 0.08425 & 0.07891 & 0.0587 \\
\hline 100 & 2 & 0 & 0 & 0 & 0 & 0 & 0 \\
\hline 150 & 3 & 0.950 & 0 & 0 & 0 & 0 & 0 \\
\hline 200 & 4 & 0 & 0 & 0 & 0 & 0 & 0 \\
\hline 250 & 5 & 0 & 0 & 0 & 0 & 0 & 0 \\
\hline
\end{tabular}

TABLE 4.24. Parameter $X 9_{h j}$ Values

\begin{tabular}{|c|c|c|c|c|c|c|c|}
\hline Release & Height & & & Energy $\mathrm{G}$ & up Ind & & \\
\hline Meters & Index & 1 & 2 & 3 & 4 & 5 & 6 \\
\hline 50 & 1 & 0.2761 & 0.3231 & 0.32880 & 0.3470 & 0.3607 & 0.3877 \\
\hline 100 & 2 & 0 & 0 & 0 & 0 & 0 & 0 \\
\hline 150 & 3 & 0 & 0 & 0 & 0 & 0 & 0 \\
\hline 200 & 4 & 0 & 0 & 0 & 0 & 0 & 0 \\
\hline 250 & 5 & 0 & 0 & 0 & 0 & 0 & 0 \\
\hline
\end{tabular}




\subsection{SURFACE WATER TRANSPORT MODELS}

Optional surface water transport models are available in the GENII computer package for estimating, at selected water usage locations, the water concentration resulting from releases of radionuclides to nontidal rivers and near-shore lake environments. The mathematical models are the same as those employed in the LADTAP II computer progran (Strenge, Peloquin, and Whelan 1986), which are based on work of Whelan as described a report by Codell, Key, and Whelan (1982). Nontidal rivers are freshwater bodies with unidirectional flow in definable channels. Near-shore lake environments are surface water areas that are near the shores of large open bodies of water and that have a straight shoreline, constant depth, and steady-state, unidirectional flow parallel to the shore.

The results of the surface water transport calculations are given in the form of a mixing ratio. The mixing ratio is the ratio of concentration at the usage location divided by the initial concentration. The ratio includes the effect of dispersion and transport. Effects of radiological decay are not considered in the surface water models but may be included separately by applying decay corrections based on transfort time.

The surface water program solves for radionuclide concentrations in a river or large lake under the following limiting assumptions (Codel1, Key, and Whelan 1982):

- constant flow depth

- constant downstream or longshore velocity

- straight river channel

- constant lateral dispersion coefficient

- continuous point discharge release of effluents

- constant river width.

Code11, Key, and Whelan (1982) note that applications of models similar to those described here should be restricted to those portions of the nearshore zone of a river or lake that are removed from the influence of the discharge. Initial dilution near the point of discharge is usually controlled by the momentum effects of jets. 
For nontidal rivers, the assumed release point is on the near shore of the river with the water plant intake point located at a downstream distance $x$ and a laterally offshore distance $y$. The water concentration (steadystate) at the downstream location is given by the following equation (NRC 1977b; Codell, Key, and Whelan 1982):

$$
C=\frac{Q_{b} C_{0}}{u d B}\left\{1+2 \sum_{i=1}^{\infty}\left[\exp -\left(\frac{i \pi}{u d B}\right)^{2} E_{y} u d^{2} x\right] \cos \left(\frac{i \pi y}{B}\right)\right\}
$$

where $\quad C=$ water concentration at the downstream location (defined by $x$ and $y)$, after continuous release for a given time period $(\mathrm{C} i / \mathrm{L})$

$B=$ constant river width $(\mathrm{m})$

$C_{0}=$ initial concentration of effluent entering the water body $(\mathrm{Ci} / \mathrm{L})$

$\mathrm{d}=$ constant flow depth (m)

$E_{y}=$ constant lateral turbulent dispersion coefficient $\left(\mathrm{m}^{2} / \mathrm{sec}\right)$

$Q_{b}=$ effluent discharge rate to the receiving water body $(\mathrm{m} 3 / \mathrm{sec})$

$u=$ average flow velocity $(\mathrm{m} / \mathrm{sec})$

$x=$ longitudinal (downstream) distance to location of interest $(\mathrm{m})$

$y=$ lateral distance to water intake point from the near shore (m).

The mixing ratio is calculated as the ratio of $C$ to $C_{0}$.

When the dilution volume is used, the water concentration is calculated as follows:

$$
C w_{j}=\frac{Q^{\prime} w_{i}}{R V}
$$

where $Q^{\prime} W_{i}$ is the release rate of radionuclide $i$ to the surface water body $(\mathrm{C} i / \mathrm{sec})$, and $V$ is the dilution volume for the receiving water body representative of the usage location $(\mathrm{L} / \mathrm{sec})$.

The dilution volume is equivalent to a river flow rate. This representative is available for use with all exposure pathways associated with contaminated water usage. 
Acute surface water releases are considered by allowing the calculation of a time-integrated water concentration, $E_{W}$, (equivalent to the $E / Q$ used in atmospheric transport) as

$$
E_{w_{i}}=\frac{Q^{\prime} w_{i}}{V} d t=\frac{Q w_{i}}{V}
$$

Near-shore lake environments are described by a model similar to the river model. The NRC (1977b) has suggested use of a quasi-steady-state model valid for simulating contaminant transport: in near-shore lake environments for distances up to 16 miles. The model is represented by the following equation:

$$
C=\left(\frac{c_{0} Q_{b}}{\left(\pi \times E_{y} E_{z}\right) \frac{1}{2}}\right) \exp \left[-\frac{y^{2} u}{4 \times E_{y}}\right] \sum_{i=-\infty}^{+\infty} \exp \left[-\frac{(2 i d-z)^{2} u}{4 \times E_{z}}\right]
$$

where $E_{z}$ is the vertical turbulent dispersion coefficient $\left(\mathrm{m}^{2} / \mathrm{sec}\right), z$ is the depth of discharge $(\mathrm{m})$, and other terms are as previously defined.

To use Equations (4.5.1) and/or (4.5.4) in any analysis, the lateral (i.e., transverse) and vertical dispersion coefficients must be defined. Dispersion coefficients should be obtained by site-specific tracer studies whenever possible. Accurately defining dispersion coefficients for all riverain or lake environments under all conditions is difficult, if not impossible at this time. However, rough estimates can be defined. The coefficients are defined such that representative properties of the water body are considered when estimating.

If the absence of site-specific estimates of dispersion coefficients (Strenge, Peloquin, and Whelan 1986) has suggested use of the following expressions:

$$
E_{y}=0.06 d u
$$

and

$$
E_{z}=0.0059 d u
$$


Even though Equations (4.5.5) and (4.5.6) were developed for rivers, they are also suggested for use with near-shore lake environments.

\subsection{SOIL CONTAMINATION MODEL}

The GENII code simultaneously considers soils in three separate areas: residential soils, non-agricultural soils, and agricultural soils. The nonagricultural soils are used only in near-field scenarios for defining parameters for arid and humid climate biotic transport. Upon the initiation of human use of the soils, the soil reverts to either residential, if the person lives there, or agricultural, if he grows crops there. Each food pathway has its own associated soil, so a large number of soil zones can be active in a single simulation. A single soil zone is made up of from one to three compartments, as illustrated in Figure 4.6 .

The surface soil is the portion on which deposition from the atmosphere and irrigation occurs, and that from which resuspension occurs. For most far-field scenarios, it is the only portion of the soil model that is used. Radionuclides may be lost from the surface soil through harvest removal, radiological decay, and leaching to deeper soil layers. In situations where subsurface contamination exists, radionuclides may be contained in waste packages or simply distributed in the soil. If the waste is in a package, it may be released to the soil using the waste package decomposition model. Radionuclides in the available subsurface soil (those not in packages) may be transported to the surface soils by root uptake by plants, by physical transport by native animals, or by human interactions through intrusion into the waste which result in redistribution of waste from deep to surface soil.

Although the model explicitly considers three compartments, the fourth is conceptually an uncontaminated layer in between the surface and deep soils. Although neglected in the model, the existence of such a layer may be easily simulated by appropriate application of rooting depth fractions (described in Section 4.7). A fraction of plant roots may be described as located in the surface soil layer, and another fraction as located in the deep soil. These two fractions may add to less than one if a clean soil layer intervenes. 


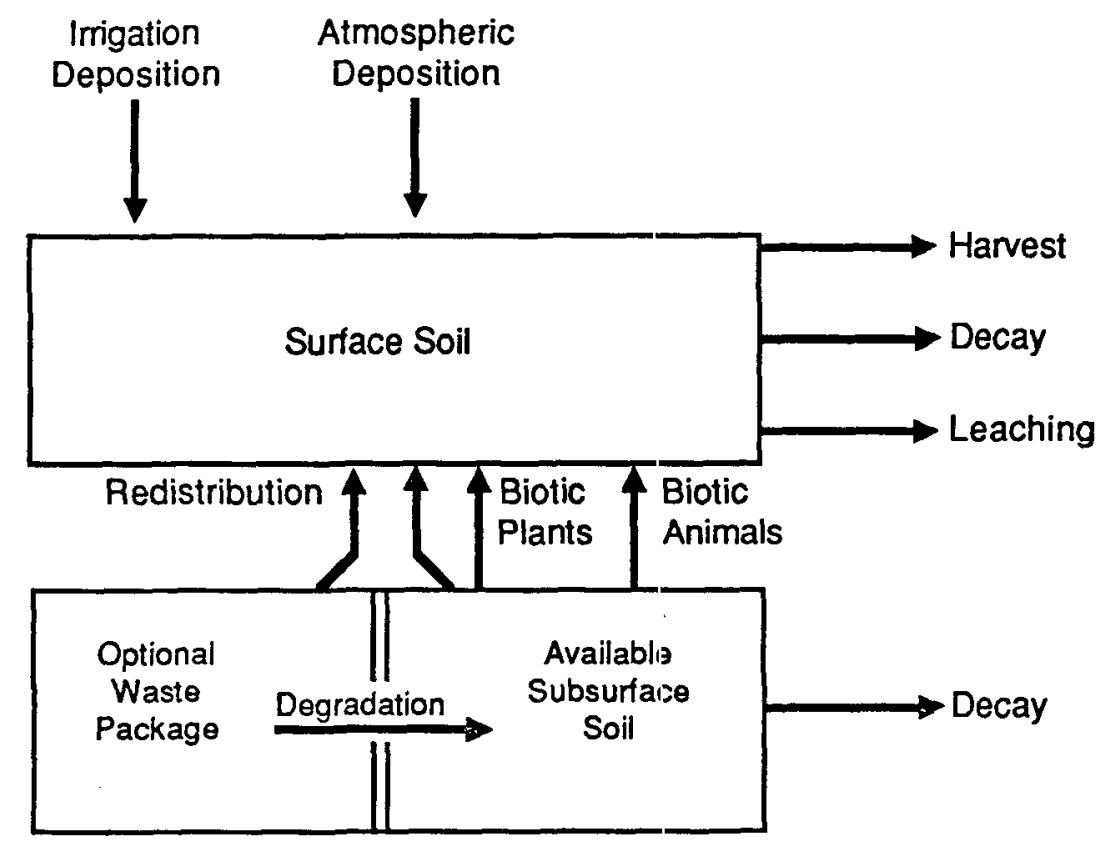

FIGURE 4.6. Schematic of Soil Compartment Interactions

\subsubsection{Deposition Rates}

For the air deposition pathway, the deposition rate, Duj, is calculated from the air concentration and an average deposition velocity as

$$
D u_{i}=C a_{i} V_{i}
$$

where $V_{d} j$ is the deposition velocity for radionuclide $(\mathrm{m} / \mathrm{sec})$.

For the water pathways, the deposition rate is calculated from the irrigation rate and water concentration as

$$
\mathrm{Du}_{i}=25.4 \mathrm{Cw} \mathrm{I} /(2.592 \cdot 106 \mathrm{MY})
$$

where

$$
I \text { = irrigation water application rate (in./yr) }
$$

$2.592 \cdot 10^{6}=$ units conversion factor $(\mathrm{sec} / \mathrm{mo})$

$25.4=$ number of liters in 1 in. applied over $1 \mathrm{~m}^{2}$

$M Y=$ number of mo/yr that irrigation occurs. 


\subsubsection{Removal Rates}

Radiological chain decay from each of the soil compartments is handled with the GENII chain decay processor. The soil compartment for each food type, animal type, and residential exposure is treated separately.

Removal from the surface soils through leaching into deeper soils is treated by means of a soil removal rate constant, $\lambda_{w i}$, which is a correction for long-term leaching of deposited radionuclides out of the surface soil rooting and resuspension zones. The values for $\lambda_{w i}$ are calculated using the formula of Baes and Sharp (1981):

$$
\lambda_{w i}=\frac{P+I-E}{d\left(1+\rho / \theta k d_{i}\right)}
$$

where

$$
\begin{aligned}
P & =\text { total precipitation }(\mathrm{cm} / \mathrm{yr}) \\
I & =\text { total irrigation }(\mathrm{cm} / \mathrm{yr}) \\
E & =\text { total evapotranspiration }(\mathrm{cm} / \mathrm{yr}) \\
d & =\text { depth of the rooting zone }(\mathrm{cm}) \\
P & =\text { soil bulk density }\left(\mathrm{g} / \mathrm{cm}^{3}\right) \\
\theta & =\text { soil volumetric water content }\left(\mathrm{mL} / \mathrm{cm}^{3}\right) \\
{k d_{j}}_{\mathbf{j}} & =\text { distribution coefficient for isotope } i(\mathrm{~mL} / \mathrm{g}) .
\end{aligned}
$$

For simplicity, the term $P+E-I$ is approximated as an overwatering term, implying about $15 \mathrm{~cm} / \mathrm{yr}$ of percolation through the rooting zone and into deeper soil layers. The depth, $d$, is defined as $15 \mathrm{~cm}$ to be compatible with other portions of the code. Baes and Sharp (1981) show that the term $\rho / \theta$ averages about 3 . Thus the soil removal constant for percolation can be considered as inversely proportional to the soil distribution coefficient, $k_{i}$. The values used in GENII are based on the most conservative (i.e., largest) value of $\mathrm{kd}_{\mathbf{j}}$ identified in a wide range of literature.

Harvest removal is simulated as a discrete process at the end of each calculational year. A quantity of each radionuclide equal to the product of the calculated vegetation concentration due to root uptake times the harvested yield (an input) is subtracted from the soil compartments. The subtraction is normalized by the root penetration factor. 


\subsubsection{Biotic Transport}

Models are included to estimate the transport of radioactivity between soil layers by plants and animals. These models were developed to estimate potential radiation dose to man resulting from biotic transport of activity at low-level radioactive waste burial sites (Mckenzie et a1. 1986). Biotic pathways include translocation by plant root systems and by burrowing insects and small mammals. Examples of plants include grass, shrubs, and trees; examples of insects and mammals include pocket mice, badgers, harvester ants, and field mice.

Transport of activity by insects and mammals is described by the following simplified model:

$$
Q s_{n i}=\sum_{j=i}^{a} C s_{n i} M_{j n}
$$

where $Q_{s_{n i}}=$ the quantity of radionuclide $i$ moved to the surface per year from soil stratum $n\left(\mathrm{Ci} / \mathrm{m}^{2} \mathrm{y} r\right)$

$a=$ number of animal species considered

$\mathrm{Cs}_{n i}=$ concentration of radionuclicle $i$ in soil stratum $n\left(\mathrm{Ci} / \mathrm{m}^{3}\right)$

$M_{j n}=$ mass of soil moved from soil stratum $n$ to the surface by animal j ( $\mathrm{m}^{3} / \mathrm{ha} \mathrm{yr}$ ).

The burrowing activity is assumed to result in excavation of soil, all of which is deposited on the surface soil layer.

The transfer of activity by plants to the surface is estimated as follows:

$$
Q P_{n i}=\sum_{l=1}^{P} C s_{n i} B v_{i} R_{l n} B l / K
$$

where $Q P_{n i}=$ quantity of radionuclide $i$ moved from soil stratum $u$ to the surface per year $\left(\mathrm{Ci} / \mathrm{m}^{2} \mathrm{yr}\right)$

$P=$ number of $p l a n t$ species considered 


$$
\begin{aligned}
\mathrm{Bv}_{\mathbf{i}} & =\text { soil-to-plant transfer factor ( } \mathrm{Ci} / \mathrm{g} \text { plant per } \mathrm{Ci} / \mathrm{g} \text { soil) } \\
\mathrm{R}_{l n} & =\text { fraction of roots of plant } l \text { in soil stratum } n \text { (dimensionless) } \\
\mathrm{B}_{l} & =\text { total biomass production of } \mathrm{plant} l \text { for a year }\left(\mathrm{g} / \mathrm{m}^{2} \mathrm{yr}\right. \text { ) } \\
K & =\text { soil density }(\mathrm{Kg} / \mathrm{m} 3) .
\end{aligned}
$$

The quantity of activity brought to the surface is assumed to be uniformly distributed in the surface soil and available for entry into plant and animal product pathways in subsequent years. Other exposures include external dose from ground surface contamination and inhalation of suspended activity.

\subsubsection{Manual Redistribution}

Transport of material from the deep soil or contained waste compartments to the surface soils may occur via human disruption of a site. This is modeled simply using a manual redistribution factor. The manual redistribution factor relates the resultant surface soil concentration, in $\mathrm{Ci} / \mathrm{m}^{2}$, to the initial subsurface concentration, in $\mathrm{Ci} / \mathrm{m}^{3}$. Because the surface soil is modeled as a slab $15 \mathrm{~cm}$ thick, a scenario in which deep soils are brought up and overlay the previous surface would use a manual redistribution factor of 0.15 (e.g., $0.15 \mathrm{~m}^{3}$ of deep soil is required to provide a $15-\mathrm{cm}$-thick layer over one $\mathrm{m}^{2}$ ). Scenarios that result in proportionately less redistribution would be simple multiples of this value.

\subsection{TERRESTRIAL EXPOSURE PATHWAYS}

This section presents the environmental transport pathway models selected to estimate exposure of individuals or populations from releases of radionuclides to air or water. The primary exposure modes considered are inhalation, external exposure, and ingestion. The exposure analysis begins with environmental concentrations predicted by air and water transport models. Output from the exposure analysis is an estimate of the effective dose equivalent for either a maximally exposed individual or the collective dose to a defined population group. Optional input is that of radionuclide concentrations in various exposure pathway media derived from environmental monitoring data. 
The mathematical models for the environmental transport pathway analysis are described in this section in general terms with emphasis given to definition of parameter requirements. For readability, activity units are show in this section as curies, although the code actually functions in "units of activity" (UOA), which are converted to becquerels or curies as required. Models are included for the major routes of transport of radionuclides from the release point to the point of intake or exposure by humans. The models used are generally of the type called the "concentration factor method" by ICRP $29(1979 b)$. The relationships between the environmental concentrations and human exposures are illustrated in Figure 2.1 on page 2.4. The four "transport" boxes on the left of the figure indicate the input to the terrestrial exposure analysis. The exposure pathways represented in this figure include the following:

- inhalation - primary mode of exposure for the air transport pathway

- drinking water ingestion - from all three water transport pathways

- aquatic food ingestion - from fish and shellfish produced in water contaminated from overland or surface water transport pathways

- crop ingestion - from farmlands contaminated by air transport and deposition, or from irrigation using water contaminated from the water transport pathways

- animal product ingestion - from animals fed contaminated crops (a11 transport pathways) or contaminated water (water transport pathways)

- external exposure to radionuclides - from contaminated air or soil (air transport pathway) and from aquatic recreational activities (swimming, boating, and shoreline activities for surface water transport pathways).

In the following discussions, the concentration of a radionuclide in air or water is assumed to be the starting point in the environmental transport analysis. For air pathways, the concentration may be represented in three forms, as follows:

1. average concentration, Cai, for time period, $t$ 
2. long-term time-integrated air concentration, $\bar{X} / Q^{\prime} \times Q^{\prime}$

3. short-term time-integrated air concentration, $E / Q \times Q$.

Each of these representations has equivalent units of $\mathrm{Ci} \mathrm{sec} / \mathrm{m}^{3}$. These units are derived for the first representation by multiplying the air concentration by the exposure period, $\mathrm{Caj}_{j} \times \mathrm{t}$.

Chronic water pathways are represented by the average water concentration, $\mathrm{CW}_{\mathrm{i}}$, for radionuclide $\mathrm{i}$ existing over the exposure period. This concentration is supplied as input to the program or is estimated from a relatively simple relationship as indicated in Section 4.5. Acute releases are represented by the time-integrated water concentration, $\mathrm{EW}$, in $\mathrm{Ci-sec/L}$. No ground water or overland water transport models are included in this program.

Mathematical models and parameters required for each model are defined for each exposure pathway in the discussions below. The dose to an individual is the output of the exposure models. For simplicity, the dose parameters are referred to as "dose". Actually, the calculations are implemented to give dose as well as effective dose equivalent to selected organs. The dose conversion factors may be defined for whatever uptake and commitment period necessary, as specified by the user, for the particular case being analyzed. The representations in the equations, therefore, do not mention the uptake or commitment periods as such, but assume that appropriate factors will be used for the particular calculation being performed.

The formulations are complete, in that they all relate directly to dose. The coded equations are, in many cases, fragmented to allow more efficient use of intermediate parameters. Some equations given here have been simplified to eliminate unit conversion factors. The actual equations as coded are given in the hand-calculation worksheets supplied in the GENII Code Maintenance Manual, Volume 3 of this set of documentation.

\subsubsection{Inhalation}

Inhalation exposure may result from inhalations of the passing plume or from inhalation of resuspended activity. The dose to an individual from 
inhalation of contaminated air is calculated from the individual ventilation rate and the air concentration parameter.

$$
\begin{aligned}
D n_{i} & =3.15 \cdot 10^{7} \text { B Caj f Dhi } \\
D n_{i} & =3.15 \cdot 10^{7} \text { B Q' } \bar{\chi} / Q^{\prime} D h_{i} \\
\text { or } \quad D n_{i} & =B Q E / Q \text { fC Dh }
\end{aligned}
$$

where $D n_{j}=$ individual dose from inhalation ( $\mathrm{rem} / \mathrm{yr}$ )

$3.15 \cdot 107=$ the number of seconds in a year

$B=$ ventilation (breathing) rate for exposed individuals $\left(\mathrm{m}^{3} / \mathrm{sec}\right)$

$\mathrm{Ca}_{\mathbf{i}}=$ average air concentration of radionuclide $\mathrm{i}\left(\mathrm{Ci} / \mathrm{m}^{3}\right)$

$D h_{i}=$ inhalation dose conversion factor for radionuclide $i$ (rem per $\mathrm{Ci}$ inhaled)

$f=$ fraction of year exposed to inhalation (dimensionless)

$E / Q=$ short-term normalized time-integrated air concentration $\left(\mathrm{sec} / \mathrm{m}^{3}\right)$

$\bar{\chi} / Q^{\prime}=$ long-term normalized time-integrated air concentration $\left(\mathrm{sec} / \mathrm{m}^{3}\right)$

$Q=$ total release of radionuclide $i(C i)$

$Q^{\prime}=$ average release rate of radionuclide $i(\mathrm{Ci} / \mathrm{sec})$

$f c=$ fraction of acute plume passage time spent in cloud (dimensionless).

Inhalation of resuspended activity uses the first form of the above representation (Equation 4.7.1a) with the air concentration estimated from either a resuspension factor or mass loading considerations. These methods are based on the assumption that the particulate matter in the air has the same activity as the soil at the location. This is represented mathematically using a resuspension factor as follows:

$$
\mathrm{Ca}_{\mathbf{j}}=\mathrm{MCs} \mathbf{i}
$$

where $M$ is the resuspension factor $\left(\mathrm{m}^{-1}\right)$ and $\mathrm{Cs}_{j}$ is the soil concentration of radionuclide $i\left(\mathrm{Ci} / \mathrm{m}^{2}\right)$. 
The resuspension factor may be estimated in one of two ways - either by using a time-dependent function developed by Anspaugh et al. (1975) or by means of a mass loading factor. The Anspaugh resuspension factor is given as:

$$
M=10^{-4} \exp (-0.15 \sqrt{ } t)+10-9
$$

where

$$
\begin{aligned}
M= & \text { resuspension factor }(m-1) \\
10-4= & \text { resuspension factor at time } t=0\left(m^{-1}\right) \\
0.15= & \text { effective decay constant controlling the availability of } \\
& \text { material for resuspension (day } 1 / 2) \\
t= & \text { time after deposition (days) } \\
10-9= & \text { resuspension factor after } 17 \text { years }(m-1) .
\end{aligned}
$$

The second term in Equation (4.7.3a), 10-9, is added based on the assumption that no further measurable decrease in the resuspension factor process occurs after about 17 years, the longest period for which there are data available.

Alternatively, the resuspension factor may be calculated from the atmospheric mass loading as

$$
M=S / \rho
$$

where $S=$ mass loading of soil in air $\left(\mathrm{g} / \mathrm{m}^{3}\right)$

$\rho=$ soil surface density $\left(\mathrm{g} / \mathrm{m}^{2}\right)$. A standard value of $224,000 \mathrm{~g} / \mathrm{m}^{2}$ to a depth of $15 \mathrm{~cm}$ is used.

Evaluation of the soil concentration was described in Section 4.6.

\subsubsection{Drinking Water Ingestion}

Exposure to radionuclides by means of the drinking water ingestion pathway may result from the ground water or surface water transport pathways. The overland transport pathway may contribute to contamination in surface water systems and thus indirectly contribute to drinking water ingestion of radionuclides (as indicated in Figure 2.1). The dose from the ingestion of water is calculated from the water concentration, water ingestion rate, a water treatment factor, and a decay correction (for transport through the 
water distribution system). The dose to an individual using a contaminated domestic water supply is calculated as follows:

$$
\begin{aligned}
& D w_{i}=U w C W_{i} T f_{j} \exp \left(-\lambda_{j} t p\right) D g_{i} \\
& D w_{i}=U w E W_{i} T f_{j} \exp \left(-\lambda_{j} t p\right) D g_{i}
\end{aligned}
$$

where $\quad D w_{i}=$ individual dose from water ingestion for radionuclide $i$ (rem/yr)

$$
\begin{aligned}
& U_{W}=\text { water-intake rate for an individual (L/yr) } \\
& \mathrm{CW}_{\mathrm{j}}=\text { water concentration of radionuclide } i(\mathrm{Ci} / \mathrm{L}) \\
& D g_{i}=\text { ingestion dose conversion factor for radionuclide } i \text { (rem per } \mathrm{Ci} \\
& \text { ingested) } \\
& T f_{j}=\text { water treatment purification factor, fraction of contaminant } \\
& \text { remaining after treatment for radionuclide } i \text { (dimensionless) } \\
& \lambda_{i}=\text { radiological decay constant for radionuclide } i\left(\mathrm{sec}^{-1}\right) \\
& \text { tp = time of transit through the water distribution system ( } \mathrm{sec}) \\
& \mathrm{EW}_{\mathbf{i}}=\text { time integrated water concentration ( } \mathrm{C} \mathrm{sec} / \mathrm{L} \text { ). }
\end{aligned}
$$

The water concentration, $\mathrm{C}_{\mathrm{i}}$, represents the concentration at the usage location. This concentration can either be provided directly as input or estimated as shown in Section 4.5 .

The water treatment purification factor accounts for removal of radionuclides during treatment in municipal water supply facilities. If no water treatment is performed, then the purification factor is 1 . The daily water intake rate is dependent on the type of calculation being performed. For an individual at maximal exposure, a higher value is generally assumed than for an average individual or population analys is. Inadvertent ingestion of water during bathing could be represented by an increment to the daily intake rate. However, the amount is considered small $(0.01 \mathrm{~L} / \mathrm{d})$ compared to the daily average intake from drinking water $(2 \mathrm{~L} / \mathrm{d})$ and is not included in this analysis. 


\subsubsection{Aquatic Food Ingestion}

Ingestion of contaminated aquatic foods is an exposure pathway considered for the surface-water transport pathways. Four types of aquatic foods are considered: fish, mollusks, invertebrates, and water plants. Fish represent organisms exposed in free-flowing rivers, lakes, or sea coasts. Mollusks and invertebrates represent organisms that live in water and feed in sediments. The radionuclide concentration in these organisms is related to the contaminant water concentration through bioaccumulation factors. The individual dose from chronic ingestion of aquatic foods is calculated for the water concentration and uptake rates as follows:

$$
D a_{j}=\sum_{f=1}^{n} U_{f} C w_{j} B_{i f} \exp \left(-\lambda_{j} \text { tp }\right) D g_{j}
$$

where $D_{a j}=$ individual dose from aquatic food ingestion for radionuclide $i$ (rem)

$\mathrm{CW}_{\mathbf{j}}=$ water concentration $(\mathrm{Ci} / \mathrm{L})$

$B_{i f}=$ bioaccumulation factor for aquatic food $f(L / k g)$

$n=$ number of aquatic food types ingested

tp $=$ time for decay from food harvest to consumption (sec)

$U_{f}=$ consumption rate of aquatic food $f$, for individuals $(\mathrm{kg} / \mathrm{d})$.

For acute exposures, for a time-integrated water concentration of Ewi, the dose is given as

$$
D a_{i}=\sum_{f=1}^{n} U_{f} E w_{j} B_{i f} \exp \left(-\lambda_{i} \text { tp) } D g_{i}\right.
$$

\subsubsection{Crop Ingestion - Chronic Exposures}

Two models are used for calculating human exposures to contaminated foods, one for chronic releases and one for acute releases. The acute model is an extension of the chronic model and is described in Section 4.7.6.

Irrigating with contaminated water or direct deposition of airborne contaminants onto plants can contaminate agricultural crops. Four food products 
associated with contaminated crop production are considered: leafy vegetables, root vegetables, grains, and fruit:. The leafy vegetable category represents plants such as lettuce whose edible portions are above ground, exposed, and eaten directly with little processing. The root vegetable category represents all other vegetable crops where direct deposition has a much smaller chance of being incorporated directly into the edible portion of the plant. The model used to estimate radionuclide concentration in edible plant portions considers uptake from two pathways: direct deposition and absorption through roots from soil. The model is a variant of that prepared for the NRC for use in Regulatory Guide 1.109 (1977a). This model has been shown to be reasonable by direct comparison of modeled and measured values (Jaquish and Napier 1987). From direct deposition onto leaves, the radionuclide concentration in the plant at time of consumption is calculated as follows:

$$
C l_{i p}=D u_{i} \operatorname{Tv}_{p} r\left[1-\exp \left(-\lambda e_{j} t e_{p}\right)\right] \exp \left(-\lambda_{i} t_{p}\right) /\left(\lambda e_{j} Y_{p}\right)
$$

where $\mathrm{Cl}_{i p}=$ concentration of radionuclide $i$ in the vegetable for pathway $p$ (leafy, root, grain, or fruit) from deposition onto leaves $(\mathrm{Ci} / \mathrm{kg})$

$D u_{j}=$ deposition rate from air or water onto farmlands $\left(\mathrm{Ci} / \mathrm{m}^{2} / \mathrm{sec}\right)$

$T v_{p}=$ translocation factor from plant surfaces to edible parts of the plant (dimensionless), currently assumed at 1.0 for leafy vegetables and forage crops, and 0.1 for all other vegetation

$r=$ fraction of initial deposition retained on the plant (dimensionless), discussed below

$\lambda e_{j}=$ effective weathering and decay constant $\left(\sec ^{-1}\right)$

te $p=$ duration of the growing period for plant type $p$ (sec)

$t_{\mathrm{p}}=\mathrm{time}$ between harvest and consumption for the vegetable type (sec)

$$
Y_{p}=\text { yield of crop type } p\left(\mathrm{~kg} / \mathrm{m}^{2}\right) \text {. }
$$

From the root uptake pathway, radionuclide concentration in the plant is calculated as follows for air deposition pathways:

$$
C r_{i p}=\left[C d_{i} R_{p 2}+\left(C s_{j} / P+C b_{j}\right) R_{p 1}\right] B v_{i p} \exp \left(-\lambda_{i} t h_{p}\right)
$$


where $\mathrm{Cr}_{\mathrm{ip}}=\mathrm{plant}$ concentration from uptake through roots for radionuclide $I$ and plant type $\mathrm{p}(\mathrm{Ci} / \mathrm{kg})$

$B v i p=$ soil-to-plant transfer factor for radionuclide $i$

(dimensionless) and plant type $p$

$C b_{j}=$ residual soil concentration from previous years' deposition of radionuclide $i(\mathrm{Ci} / \mathrm{kg})$

$C s_{i}=$ area soil concentration from air deposition for radionuclide

- i $\left(\mathrm{Ci} / \mathrm{m}^{2}\right)$, discussed below

$\mathrm{Cd}_{\mathrm{i}}=$ deep soil concentration for radionuclide $\mathrm{i}, \mathrm{Ci} / \mathrm{kg}$, discussed below

$P=$ effective soil density in the plow layer $\left(\mathrm{kg} / \mathrm{m}^{2}\right)$

$R_{p 1}=$ root penetration factor for surface soil, (dimensionless)

$R_{p 2}=$ root penetration factor for deep soil, (dimensionless).

This root penetration factor is included for special cases where the contamination is not uniform with soil depth. See the soil model discussion in Section 4.6. The factor allows a simple correction based on the fraction of the plant room system that is in contaminated soil. For example, if the contaminated soil is buried a meter or so below the surface, only a portion of the root system would reach the contamination. Uptake of radioactivity for such cases would likely be less than if the entire root system were in contact with the contamination.

The total plant concentration at the time of consumption is then calculated as the sum of the contributions from direct deposition and soil uptake as follows:

$$
C_{i p}=C l_{i p}+C r_{i p}
$$

where $C_{i p}$ is the concentration of radionuclide $i$ in crop type $p$ at the time of consumption $(\mathrm{Ci} / \mathrm{kg})$.

The individual dose from ingestion of agricultural crops is estimated from the plant concentration in Equation (4.7.8) and the average consumption rate of vegetables as follows: 


$$
D v_{i}=\sum_{p=1}^{4} U_{p} C_{i p} D g_{i}
$$

where $D v_{i}$ is the dose from consumption rate of contaminated crops (rem/yr), . and $U_{p}$ is the consumption rate of crop type $p$ (leafy, root, grain, or fruit) in $\mathrm{kg} / \mathrm{yr}$. The summation is over the four vegetable types.

\section{Interception Fraction, $r$}

The interception fraction, $r$, for a given vegetation type is a factor that accounts for the fact that not all of the material deposited within a unit area will end up on vegetation surfaces. The fraction of the total deposition that initially resides on veget:ation is the interception fraction, $r$, such that $0<r<1.0$.

The interception of materials in irrigation water is not well studied. Thus, a default value of 0.25 is used for all materials deposited on all vegetation types by irrigation.

An empirical relationship between bicmass and interception fraction was originally suggested by Chamberlain (1967). This model has been expanded recently by Pinder, Ciravolo, and Bowling (1988) for grasses and other species. The following form of the equation is used for grasses, leafy vegetables, and grains:

$$
r=1.0-\exp \left(-2.9 Y_{p} f_{d}\right)
$$

A similar equation is used for fruits and other vegetables:

$$
r=1.0-\exp \left(-3.6 Y_{p} f_{d}\right)
$$

where $f_{d}$ is the dry-to-wet biomass ratio, and $Y_{p}$ is the standing biomass of the growing vegetation, $\mathrm{kg}(\mathrm{wet}) / \mathrm{m}^{2}$. The dry-to-wet ratio is required because the Pinder formulations are given in terms of dry biomass. This formulation results in the need to define the growing biomass, as well as the harvested yield (for use with the harvest removal term defined in Section 4.6). The values currently in the GENII default files are given in Table 4.25. 
The value formerly used at Hanford for the interception fraction was 0.25 for atmospheric deposition and for irrigation deposition. The newer formulations for atmospheric deposition tend to produce higher concentrations than did previous techniques.

\subsubsection{Animal Product Ingestion - Chronic Exposures}

Atmospheric deposition of contaminants onto feed crops and use of contaminated water to irrigate feed crops can result in the ingestion of contaminated crops by animals. In addition, contaminated water can be used for animal drinking water. Four animal products are considered: beef, poultry, cow milk, and eggs. In evaluating the radionuclide concentration in the beef, poultry, milk, and eggs, the animals are assumed to be feed crops containing radionuclide levels defined by Equation (4.7.8), without the decay correction between harvest and consumption [the exponential term with thp in Equations (4.7.6) and (4.7.7)]. The animal product concentration resulting from animal ingestion of contaminated feed is calculated as follows:

$$
C_{i m}=C_{i p} F_{m i} f Q f \exp \left(-\lambda_{j} t h_{m}\right)
$$

where $\mathrm{Cf}_{\mathrm{im}}=$ concentration of radionuclide $\mathrm{i}$ in animal product $\mathrm{m}$, from animal ingestion of contaminated feed ( $\mathrm{Ci} / \mathrm{L}$ for $\mathrm{milk}$ and $\mathrm{Ci} / \mathrm{kg}$ for meat)

$C_{i p}=$ concentration of radionuclide $i$ in feed crop $p$, used by the animal ( $\mathrm{Ci} / \mathrm{kg}$ )

$f=$ fraction of animal feed that is contaminated (dimensionless)

$\mathrm{F}_{\mathrm{mi}}=$ transfer coefficient that relates daily intake rate by an animal to the concentration in an edible animal product ( $\mathrm{Ci} / \mathrm{L}$ milk per $\mathrm{Ci} /$ day for milk and $\mathrm{Ci} / \mathrm{kg}$ meat per $\mathrm{Ci} /$ day for meat)

Qf = consumption rate of feed by the animal $(\mathrm{kg} / \mathrm{d})$

$t h_{m}=$ holdup time between harvest or slaughter and consumption for the animal product $(\mathrm{sec})$. 
TABLE 4.25. Harvested Yield, Standing Biomass, and Ratio of Dry to Wet Vegetation Used in Interception and Harvest Removal Models

\begin{tabular}{|c|c|c|c|}
\hline Category & $\begin{array}{r}\text { Harvested(a) } \\
\text { Yield (wet) } \\
\text { for Soil Removal } \\
\end{array}$ & $\begin{array}{c}\text { Standing (b) } \\
\text { Biomass (wet) } \\
\text { for Interception }\end{array}$ & $\begin{array}{l}\text { Dry-to-Wet (c) } \\
\text { Ratio for } \\
\text { Interception }\end{array}$ \\
\hline Leafy Vegetables & 2.0 & 2.0 & 0.10 \\
\hline Other Vegetables & 4.0 & 2.0 & 0.25 \\
\hline Grain & 0.6 & 0.8 & 0.18 \\
\hline Fruit & 2.0 & 3.0 & 0.18 \\
\hline Meat: Forage/Hay & $2.0(d)$ & 1.0 & 0.20 \\
\hline Meat: Grain & 0.6 & 0.8 & 0.18 \\
\hline $\begin{array}{l}\text { Poultry \& Eggs: } \\
\text { Grain }\end{array}$ & 0.6 & 0.8 & 0.18 \\
\hline Milk: Fresh Forage & $3.0(d)$ & 1.5 & 0.20 \\
\hline Milk: Hay & $2.0(d)$ & 1.0 & 0.20 \\
\hline
\end{tabular}

\footnotetext{
(a) Adapted from data in Shor, Baes, and Sharp (1982) for the Washington counties of Benton, Franklin, and Yakima.

(b) Derived from data in Shor, Baes, and Sharp (1982), Strenge, Bander, and Soldat (1987), or Napier et al. (1980).

(c) Adapted from Till and Meyer (1983), Section 5.

(d) Multiple cuttings.
}

In evaluating the feed concentration, $C_{i p}$, from Equation (4.7.8), parameters representative of animal feed production are used which differ from vegetable production parameters for human consumption. For example, the growing period is usually set to 30 days to represent animal grazing habits.

The contribution to animal product concentration from animal ingestion of contaminated water is calculated as follows:

$$
C_{W_{i m}}=C_{W_{i}} F_{m i} f_{W} Q_{w} \exp \left(-\lambda_{i} t_{m}\right)
$$

where $\mathrm{C}_{\mathrm{im}}=$ concentration of radionuclide $i$ in an animal product $m$, from animal ingestion of water ( $\mathrm{C} / \mathrm{kg}$ for meat and $\mathrm{Ci} / \mathrm{L}$ for $\mathrm{milk}$ ) $\mathrm{fw}_{\mathrm{w}}=$ fraction of animal water that: is contaminated (dimensionless) $Q w=$ consumption rate of water by the animal (L/d). 
Default values of the animal consumption rates used in the GENII code are given in Table 4.26 .

The total concentration in the animal product is the sum of the contributions from feed and water intake:

$$
c_{i m}=C_{i m}+C_{i m}
$$

where $\mathrm{C}_{\mathrm{im}}$ is the concentration of radionuclide $\mathrm{i}$ in animal product $\mathrm{m}(\mathrm{Ci} / \mathrm{kg})$ for beef and eggs, and $\mathrm{Ci} / \mathrm{L}$ for $\mathrm{milk}$ ).

The individual dose is calculated from the animal product concentration and the consumption rate of the products as follows:

$$
D_{m i}=\sum_{m=1}^{4} U_{m} C_{i m} D g_{i}
$$

where $D_{m i}$ is the individual dose for radionuclide $i$ from ingestion of animal product $m(r e m)$, and $U_{m}$ is the average daily consumption rate of animal product $\mathrm{m}$ ( $\mathrm{L} / \mathrm{d}$ for $\mathrm{milk}$ and $\mathrm{kg} / \mathrm{d}$ for meat). The summation is over the four animal product types - meat, milk, poultry, and eggs.

TABLE 4.26. Default Consumption Rates of Feed

\begin{tabular}{|c|c|c|}
\hline Animal & $\begin{array}{c}\text { Feed or Forage } \\
(\mathrm{kg} / \text { day })\end{array}$ & $\begin{array}{l}\text { Water } \\
\text { (L/day) }\end{array}$ \\
\hline Milk Cow & 55 & 60 \\
\hline Beef Cattle & 68 & 50 \\
\hline Poultry & 0.12 (dry feed) & 0.3 \\
\hline
\end{tabular}
and Water by Farm Animals

Adapted from Baker, Hoenes, and Soldat (1976).

\subsubsection{Terrestrial Food Pathways Models - Acute Releases}

Radionuclides deposited on farmland can result in human radiation exposure through farm product pathways. These pathways include food crops 
and animal products. Under accidental release conditions, the amount of activity ingested through these pathways is difficult to assess. The annual average parameter values and equilibrium bioaccumulation factors used in the chronic pathway analysis models are not directly applicable in describing radionuclide behavior during the short tine periods considered in accidental release situations, but they may be more useful for estimating timeintegrated dose. The GENII formulation for acute releases takes advantage of this. Information is not provided on close rates, but estimates are given for total integrated dose for specific set:s of assumptions. The GENII formulation is similar to, but an extension of, that used in the Belgian mode1 DOSDIM (Govaerts, Martens, and Kretzichmer 1983). The GENII formulation is also conceptually similar to that used in the model PATHWAY (Whicker and Kirchner 1987), but it uses fewer input parameters.

The GENII formulation for acute releases is intended to be used in prospective assessments, for which the exact timing and conditions of an accident cannot be predicted. For conditions following an accident, the GENII models using known input concentrations should instead be used.

The basic input to the following derivation is season of year, which is actually a surrogate for a more complex set of assumptions concerning crop status and time until harvest. In brief:

- Winter accidents result in soil contamination which becomes a source of contamination via root uptake for all plant pathways for the remainder of the year.

- Spring accidents result in soil contamination for root vegetables, grains, and fruit (because of little plant development and long weathering times). They also result in deposition on leafy vegetables and pasture grass (thus causing milk and beef contamination). Human exposures to ingested contaminants via the leafy vegetable and milk pathways are moderated by the weathering of the initial contamination, using the model developed for the PABLM code by Napier (Till and Meyer 1983). A weathering time of up to three months is assumed. 
- Summer accidents result in both soil and leaf contamination of all vegetable products and of forage for animals. A weathering time of one month is assumed for root vegetables, grains, and fruit; immediate harvest and use are assumed for leafy vegetables and forage using the PABLM continuous weathering model.

- Autumn accidents are assumed to occur immediately before harvest, resulting in no soil uptake for crops for that year.

The initiating parameter in all of the following equations is the initial deposition on the ground and plant surfaces. This may be defined from the integrated air concentration or the integrated water concentration as

$$
\begin{aligned}
& C s(t=0)_{a}=E / Q Q V_{d} \\
& C s(t=0)_{W}=E_{W} I
\end{aligned}
$$

where $\operatorname{cs}(t=0) a=$ the initial soil surface concentration (i.e., at time $t=0$ ) from atmospheric deposition, also interpreted as the total downward flux of material per unit area $\left(\mathrm{Ci} / \mathrm{m}^{2}\right)$

$C s(t=0)_{W}=$ the initial soil surface concentration from irrigation deposition $\left(\mathrm{Ci} / \mathrm{m}^{2}\right)$

and other terms are as defined in prior sections.

The basic formulation for human intake of contaminated crops is dependent on the times of deposition, harvest, and consumption. The initial concentration on the plant can be expressed as

$$
C_{p}(t=0)=r C_{s}(t=0) T v_{p} / Y
$$

The initial plant concentration due to the initial deposition decreases over time by means of radiological decay and weathering. These processes are assumed to occur continuously from deposition to harvest. The plant concentration at harvest is calculated as

$$
C_{p}\left(t=T_{h}\right)=C_{p}(t=0) e^{-\lambda e} T h
$$


where $\lambda e=$ an effective removal constant

$\lambda e=\lambda r+\lambda w$

$\lambda r=$ radiological decay constant

$\lambda w=$ weathering removal rate, based on a half time of 14 days.

After harvest, a delay period usually occurs before consumption of the food product begins. During this delay, additional radiological decay may occur. For some crops, the consumption period may last for weeks or months. To estimate the uptake during this period "consumption is assumed to occur at a uniform rate. The total intake is then given by a time integral over the consumption period

$$
I=\int_{0}^{T} C_{p}\left(t=T_{h}\right) e^{-\lambda r t} U d t
$$

or

$$
I=U C_{p}\left(t=T_{h}\right)\left[1-e^{-\lambda r} T_{f}\right] / \lambda r
$$

where $\quad I=$ the total activity of a radicnuclide ingested over a consumption period Tf ( $\mathrm{C} i$ )

$U=$ the average daily ingestion rate of the crop over the ingestion period ( $\mathrm{kg} /$ day)

$T_{f}=$ the length of the uptake period (day).

The radiation dose received by a person ingesting this amount of activity can be determined using appropriate ingestion dose conversion factors.

This model may be extended for use with animal products. The animal is assumed to eat contaminated crops or drink contaminated water and produce contaminated products continuously over the consumption period defined for humans. The animal product concentration at the time of production is then given as

$$
C_{a}(t)=C_{p}(t) F_{m i} f Q f
$$

This animal concentration is not strictly appropriate, because the equilibrium constant does not directly apply to the transient case; but the integral 
of the concentration is appropriate. The result for the animal product concentration is then used in Equation (4.7.19b) to estimate the total radionuclide intake by an individual consuming the particular animal product.

This model is most appropriate for animal products in which the radionuclide concentration comes to a rapid equilibrium.

Application of the general equations is illustrated in Tables 4.27 through 4.30. These tables present the equations used for leafy vegetables, other vegetables, milk and meat, and other animal products for the four seasons. The equations yield the total time-integrated concentration realized by the consumer of the food product, in $\mathrm{Ci}-\mathrm{yr} / \mathrm{kg}$, which when multi$\mathrm{plied}$ by the consumption rate in $\mathrm{kg} / \mathrm{yr}$ gives in $\mathrm{Ci}$ the total intake of radionuclides resulting from the initial release.

For each of the equations in Tables 4.27 through 4.30 , terms are included for uptake to the crop by means of root and leaf pathways. For winter and some spring pathways, the leaf route is set to zero. The plant is assumed dead above ground during winter. For autumn accidents, the root pathways are set to zero because the harvest is assumed to occur soon enough after the deposition that the plant has not come to an equilibrium with the soil. Most equations also explicitly contain the exponential term of Equation (4.7.19b). Those that do not, contain the term $[1 /(\lambda r+\lambda w)]$. This is the exponential integrated to infinity, because the weathering term is so fast that the remainder of the integral term is negligible.

\subsubsection{External Exposure}

Land and surface-water transport pathways are involved in external radiation exposure. Individuals may be exposed to contaminated air and ground. They may also be exposed through aquatic recreational activities, such as boating, swimming, and shoreline water use. The radiation dose is calculated as follows from the water concentration or soil concentration, depending on which transport pathway is being studied. 


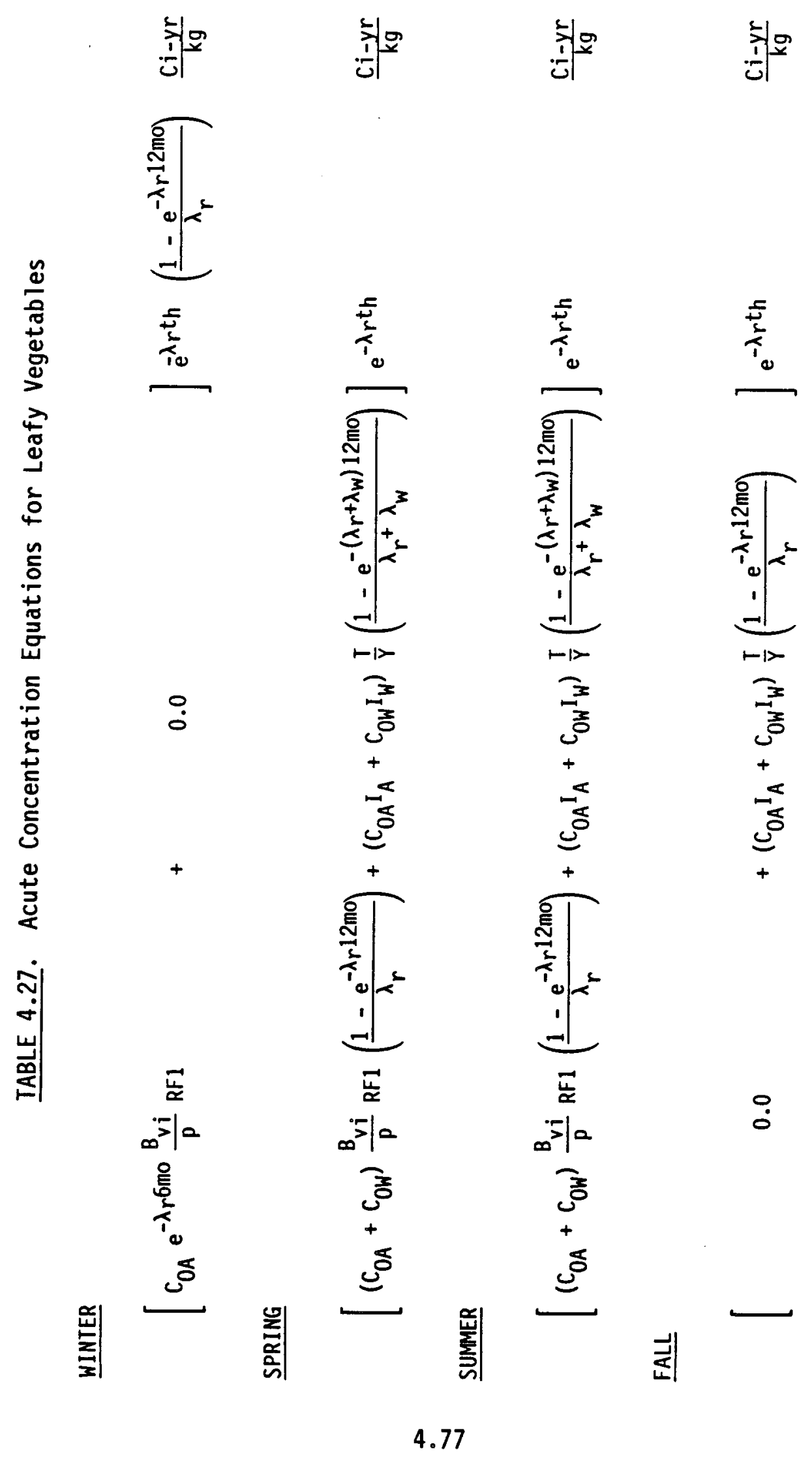




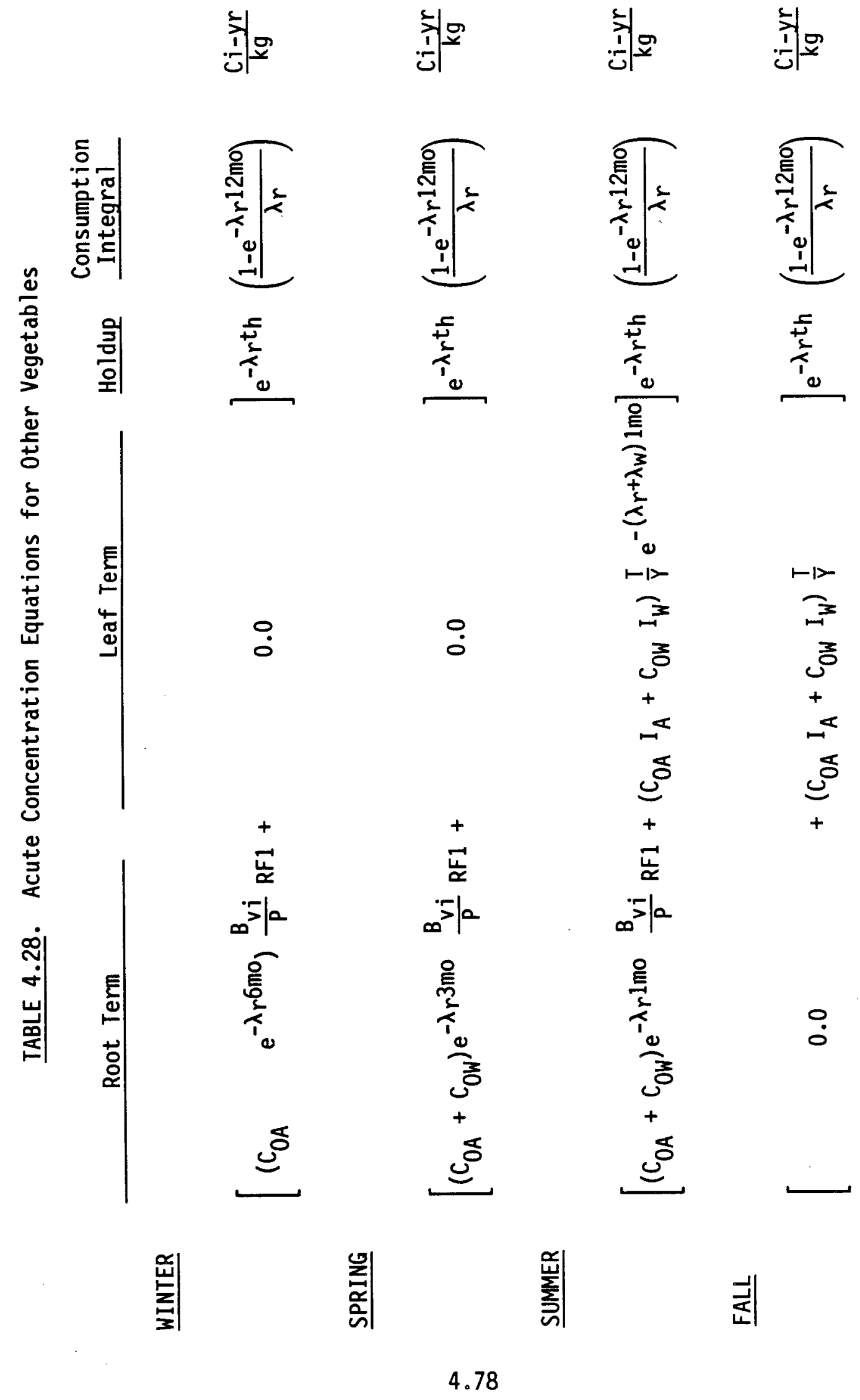


TABLE 4.29. Acute Deposition Integrated Concentration Equations for Milk and Beef

WINTER

Forage Equation:

Root

Leaf

$\left(\left(C_{0 A} e^{-\lambda r 6 m o} \frac{B V i}{p} R F 1\right) F R+0.0=A\right.$

Stored Feed:

Root Leaf

$\therefore \quad\left(\left(C_{O A} e^{-\lambda r 6 m o} \frac{B V i}{p} R F 1\right)(1-F R)+0.0=B\right.$

Hater:

$Q_{w} E_{w} T_{f}=C$

Final Equation:

$\left\{(A+B)\left(\frac{1-e^{-\lambda r 12 m o}}{\lambda_{r}}\right) Q f+C\right\} F_{m i} e^{-\lambda_{r} t h}$
SPRING

Forage Equation:

Root

Leaf

$\left[\left(C_{O A}+C_{O W}\right) \frac{B V i}{P} \operatorname{RF} 1\left(\frac{1-e^{-\lambda_{r} 12 m g}}{\lambda_{r}}\right)+\left(C_{O A} I_{A}+C_{O W} I_{W}\right) \frac{T}{Y}\left(\frac{1-e^{-\left(\lambda_{r}+\lambda_{W}\right) 12 m 0}}{\left(\lambda_{r}+\lambda_{W}\right)}\right)\right] F R=A$

Stored Feed:

Root

Leaf

$\left[\left(C_{O A}+C_{O W}\right) e^{-\lambda r^{3 m o}} \frac{B V i}{p} R F 1+0.0\right](1-F R)\left(\frac{1-e^{-\lambda_{r} 12 m g}}{\lambda_{r}}\right)=B$

Hater:

$Q_{w} E_{w} T_{f}=C$

Final Equation:

$\{(A+B) a f+C\} F_{m i} e^{-\lambda_{r} t_{h}}$ 
TABLE 4.29. (contd)

SUMMER

Forage Equation:

Root

leaf

$\left[\left(C_{O A}+C_{O W}\right) \frac{B V I}{p} R F I\left(\frac{1-e^{-\lambda_{r} 12 m o}}{\lambda_{r}}\right)+\left(C_{O A} I_{A}+C_{O W} I_{W}\right) \frac{T}{Y}\left(\frac{1-e^{-\left(\lambda_{r}+\lambda_{W}\right) 12 m o}}{\left(\lambda_{r}+\lambda_{W}\right)}\right)\right] F R=A$

Stored Feed:

$\underline{\text { Root }}$

Leaf

$\stackrel{\infty}{O} \quad\left[\left(C_{O A}+C_{O W}\right) e^{-\lambda r 1 m 0} \frac{B V i}{P} R F 1+\left(C_{O A} I_{A}+C_{O W} I_{W}\right) \frac{T}{Y} e^{-\left(\lambda_{r}+\lambda_{W}\right) 1 m O}\left(\frac{1-e^{-\lambda_{r} 12 m O}}{\lambda_{r}}\right)(1-F R)\right]=B$

Water:

$Q_{w} E_{w} T_{f}=C$

Final Equation:

$\{(A+B) Q f+C\} F_{m i} e^{-\lambda_{r} t_{h}}$
FALL

Forage Equation:

Root Leaf

$\left[0.0+\left(C_{O A} I_{A}+C_{O H} I_{W}\right) \frac{T}{Y}\left(\frac{1-e^{-\lambda_{r} 12 m g}}{\lambda_{r}}\right)\right] F R=A$

Stored Feed Equation:

$\left[0.0+\left(C_{O A} I_{A}+C_{O W} I_{W}\right) \frac{T}{Y}\left(\frac{1-e^{-\lambda_{r} 12 m o}}{\lambda_{r}}\right)\right](1-F R)=B$

Hater:

$Q_{w} E_{w} T_{f}=c$

Final Equation:

$\{(A+B) Q f+C\} F_{m i} e^{-\lambda r t h}$ 
TABLE 4.30. Acute Deposition Integrated Concentration Equations for Other Animals

Grain Roots $\quad$ Grain Leaves

Consumption

Integral

Water Term

Holdup

WINTER

$$
\left\{\left[\left(C_{O A} \quad e^{-\lambda r 6 m o} \frac{B_{v i}}{P} R F 1+\quad 0.0 \quad\right] Q_{f}\left(\frac{1-e^{-\lambda_{r} 12 m o}}{\lambda_{r}}\right)+Q_{W} E_{W} T_{f}\right\}_{F I} e^{-\lambda_{r} \text { th }}\right.
$$

SPRING

$\stackrel{\leftrightarrow}{\dot{\infty}} \quad\left\{\left[\left(C_{O A}+C_{O W}\right) e^{-\lambda r 3 m o} \frac{B_{v i}}{P} R F 1+\quad 0.0 \quad\right] Q_{f}\left(\frac{1-e^{-\lambda_{r} 12 m o}}{\lambda_{r}}\right)+Q_{W} E_{W} T_{f} P A\right\} F M I e^{-\lambda_{r} t h}$

SUMMER

$$
\left\{\left[\left(C_{O A}+C_{O W}\right) e^{-\lambda_{r} 1 m o} \frac{B_{V i}}{P} R F 1+\left(C_{O A} I_{A}+C_{O W} I_{W}\right) \frac{T}{\gamma} e^{-\left(\lambda_{r}+\lambda W\right) 1 m o}\right] Q_{f}\left(\frac{1-e^{-\lambda_{r} 12 m o}}{\lambda_{r}}\right)+Q_{W} E_{W} T_{f} \quad F_{M I} e^{-\lambda_{r} t h}\right.
$$

FALL

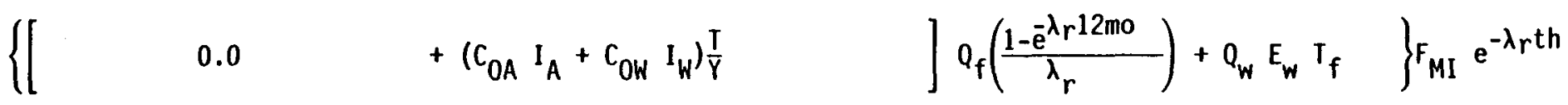


Aquatic Recreational Activities

For water pathways, the recreational dose is

$$
D e_{j}=(0.5 t b+t s) C W_{j} D b_{j}+t f C_{j} W D s i
$$

where $\mathrm{De}_{j}=$ dose to an individual from external exposure for radionuclide $i$, from aquatic recreational activities (rem)

$C d_{j}=$ sediment concentration of radionuclide $i$, deposited on shoreline from contaminated water $\left(\mathrm{Ci} / \mathrm{m}^{2}\right)$

tb = time spent by an individual in boating ( $h$ )

$t f=$ time spent by an individual fishing or in shoreline activities (h)

ts = time spent by an individual swimming $(h)$

$\mathrm{Db}_{j}=$ external dose conversion factor for radionuclide $i$, for immersion in water ( $\mathrm{rem} / \mathrm{h}$ per $\mathrm{Ci} / \mathrm{L}$ )

Ds $j$ = external dose conversion factor for exposure to a contaminated plane of radionuclide $i$ (rem/h per $\mathrm{Ci} / \mathrm{m}^{2}$ )

$W=$ shore width factor to correct for finite size of shoreline (dimensionless).

The average sediment concentration is estimated from a model developed by Soldat, Robinson, and Baker (1974) relating water concentration to sediment concentration following a long period of deposition. The sediment concentration is given by the following expression:

$$
C_{d}=100 T_{i} C_{w i}\left[1-\exp \left(-\lambda_{i} T_{b}\right)\right]
$$

where $T_{b}=$ length of time the sediment is exposed to the contaminated water (d)

$T_{i}=$ physical half-life of the radionuclide (d)

$100=$ transfer constant from water to sediment $\left(L / \mathrm{m}^{2} / \mathrm{d}\right)$.

The value of the transfer constant was derived for several radionuclides by using data obtained from analyzing water and sediment samples taken from the Columbia River between Richland, Washington, and the river mouth, and from Tillamook Bay, Oregon, $75 \mathrm{~km}$ south of the river mouth (Nelson 1965; 
Toombs and Cutler 1968). The equation estimates an effective surface contamination for calculating gamma exposure rates to persons standing on sediment.

The shore-width factor, $W$, represents the fraction of dose from an infinite plane source an individual could receive from a given shoreline situation that may not be well described as an infinite plane (for which the dose factors are defined). The shore-width factor is essentially a geometric correction. Suggested values for $W$ are derived from experimental data (Dunster 1971) and are presented in Table 4.31.

TABLE 4.31. Suggested Values; for Shore-Width Factor

Shoreline Type

River Shoreline

Lake Shore

Nominal Ocean Site

Tidal Basin
Shore-Width Factor

0.2

0.3

0.5

1.0

For acute releases, the time-integrated water concentration, $E_{W}$, is used in place of the integral portion of Equation (4.7.22a) as

$$
C_{d i}=100 T_{j} E_{W}
$$

Exposures from sediment are then calculated as given in Equation (4.7.21). For swimming and boating, certain assumptions have been incorporated. It is assumed that maximum individuals are exposed to the entire passage of the contaminated water. Populations, conversely, are assumed to completely avoid contamination. These assumptions are only valid if the acute release and transport times are short. Otherwise, the individual dose could be substantially over estimated. With these assumptions, Equation (4.7.21) can be rewritten as

$$
D e_{i}=1.5 E w_{i} D b_{i}+t f C_{j} W D s_{i}
$$




\section{Contaminated Land}

Generally, exposure to contaminated ground is important only for the air transport pathway because water transport pathways will not result in widespread contamination. Airborne deposition is assumed to cover the entire region of the defined population group, and all individuals are potentially exposed. However, in evaluating the maximally exposed individual dose, soil contamination from irrigation is also included as an external exposure route. The radiation dose is calculated from the soil concentration as follows:

$$
D x_{i}=t_{s} C_{i} D s_{i}
$$

where $D i$ is dose to an individual from exposure to contaminated ground for radionuclide $i$ (rem), and ts is the time of exposure to contaminated ground (h).

\section{Inadvertent Ingestion of Soil and Water}

Uptake of radionuclides may result from inadvertent ingestion of soil with foods or of water during recreational swimming. Soil ingestion is estimated conservatively using soil ingestion amounts of Kimbrough et al. (1983) presented in Table 4.32. Using the values in this table, an estimate of average soil ingestion over the lifetime of an individual can be obtained. This average value is $410 \mathrm{mg} / \mathrm{d}$. The average individual dose from inadvertent ingestion of soil is then estimated as follows:

TABLE 4.32. Soil Ingestion by Age(a)

Age Group Soil Ingested (mg/d)

$\begin{array}{cr}0-9 \mathrm{mo} & 0 \\ 9-18 \mathrm{mo} & 1,000 \\ 1.5-3.5 \mathrm{yr} & 10,000 \\ 3.5-5 \mathrm{yr} & 1,000 \\ >5 \mathrm{yr} & 100\end{array}$

(a) Derived from Kimbrough et al. (1983). 


$$
D d_{j}=410 \operatorname{td~Csi~} D g_{i} / 2.24 \cdot 108
$$

where $D d_{i}=$ dose to an individual from inadvertent ingestion of soil (rem) $t d=$ period of exposure to ingestion of soil (d) $410=$ daily average soil ingestion rate (mg soil/d) $2.24 \cdot 10^{2}$ = areal soil density (mg soil/ $\left./ \mathrm{m}^{2}\right)$.

Inadvertent ingestion of water may also occur during recreational swimming. The amount ingested is assumed to be $10 \mathrm{~mL}$ per hour of swimming. The time of swimming (ts) as defined above for Equation (4.7.21) is used to estimate the average daily exposure as follows:

$$
D r_{j}=0.01 C_{j} \text { ts } D_{j}
$$

where $D r_{i}$ is the dose to an individual from ingestion of water during recreational swimming (rem), and 0.01 is the inadvertent water ingestion rate during recreational swimming (L/hr).

\subsubsection{Population Dose Calculations}

The above models have described calculation of dose to individuals. Population doses can be estimated from the individual dose in two ways, depending on the basis for determining the exposure of the population group. If the number of people exposed by a given pathway is known, then the population dose for that pathway is estimated as the product of individual dose and population exposed as

$$
D_{p i}=P_{p} D_{p i}
$$

where $P_{p i}=$ population dose for pathway $p$ and radionuclide $i$ (person-rem) $D_{p i}=$ dose to an individual from pathway $p$ and radionuclide $i$ (rem)

$P_{p}=$ number of people exposed by pathway $p$ (persons).

External exposure pathways and inhalation exposure use Equation (4.7.27). The ingestion pathway may also use Equation (4.7.27) if the amount of food produced is known to be sufficient to feed the given population. Or alternatively, base the number of people exposed on the amount of 
food produced. This is accomplished by considering the number of people that can be fed by the production using average ingestion rates.

$$
P_{p}=K_{p} /\left(U_{p} t_{p}\right)
$$

where $P_{p}=$ population served by production of food type $p$ (persons)

$K_{p}=$ production of food type $p$ during period of exposure $(\mathrm{kg}$ or $\mathrm{L})$

$U_{p}=$ consumption rate of food type $p$ during the period of exposure $(\mathrm{kg} / \mathrm{d}$ or $\mathrm{Ld})$

$t_{p}=$ period of exposure (d).

The population served as calculated by Equation (4.2.28) is then used in Equation $(4.7 .27)$ to estimate the population dose.

\subsubsection{Special Models for $3 \mathrm{H}$ and $14 \mathrm{C}$}

The behavior of the radionuclides tritium and carbon-14 in exposure pathways is handled in a special manner. The concentrations of tritium or carbon-14 in environmental media (soil, plants, and animal products) are assumed to have the same specific activity (curies of radionuclide per kilogram of soluble element) as the contaminating medium (air or water). The fractional content of hydrogen or carbon in a plant or animal product is then used to compute the concentration of tritium or carbon-14 in the food product under consideration. The hydrogen content in both the water and the nonwater (dry) portion of the food product are used when calculating the tritium concentration. For airborne releases, it is assumed that plants obtain all their carbon from airborne carbon dioxide and that animals obtain all their carbon through ingestion of plants.

The transfer of $14 \mathrm{C}$ from water to plants is difficult to model because plants acquire most of their carbon from the air. Currently available models for carbon-14 uptake by plants from water use specific-activity models relating the activity in the plants directly to the activity in irrigation water. This is extremely conservative in that it assumes that plants receive all of their carbon from water. An interim model is described here based on the ratio of grams of carbon-14 to grams of total carbon in soil and a correction for the amount of carbon plants obtain from soil. 
The special models recommended for $3 / H$ are described first followed by the ${ }^{14} \mathrm{C}$ models. The concentration of tritium in vegetation is calculated as

$$
C_{H p}=9 C_{W H} F_{H p}
$$

where $C_{H p}=$ the concentration of $3 \mathrm{H}$ in plant type $\mathrm{p}(\mathrm{Ci} / \mathrm{kg})$

$\mathrm{CWH}_{\mathrm{WH}}=$ the concentration of tritium in the environmental water $(\mathrm{Ci} / \mathrm{L})$

$\mathrm{FHp}_{\mathrm{Hp}}=$ the fraction of hydrogen in total vegetation.

The coefficient 9 converts tritium concentration in environmental water to concentration in hydrogen. The fractions of hydrogen in various food types are given in Table 4.33 .

For airborne release, the concentration of $3 \mathrm{H}$ in environmental water is calculated from the air concentration and the absolute humidity as follows:

$$
\mathrm{CWH}_{\mathrm{H}}=\mathrm{CaH} / \mathrm{H}
$$

where $\mathrm{CaH}_{\mathrm{H}}$ is the average air concentration of $3 \mathrm{H}\left(\mathrm{Ci} / \mathrm{m}^{3}\right)$, and $\mathrm{H}$ is the absolute humidity $\left(\mathrm{L} / \mathrm{m}^{3}\right)$, taken to be $0.008 \mathrm{~L} / \mathrm{m}^{3}$ for Hanford.

The concentration of tritium in the animal product is

$$
C_{H m}=\frac{C_{H p} Q f+C_{H} Q w}{F_{H f} Q f+Q w / 9} F_{H m}
$$

where $\mathrm{CHm}_{\mathrm{m}}=$ concentration of $3 \mathrm{H}$ in animal product $\mathrm{m}(\mathrm{Ci} / \mathrm{kg}$ or $\mathrm{Ci} / \mathrm{L})$

$\mathrm{CHp}_{\mathrm{Hp}}=$ concentration of $3 \mathrm{H}$ in animal product feed $\operatorname{crop} \mathrm{p}(\mathrm{Ci} / \mathrm{kg})$

$\mathrm{CWH}_{\mathrm{H}}=$ concentration of $3 \mathrm{H}$ in animal drinking water $(\mathrm{Ci} / \mathrm{L})$

$F_{H f}=$ fraction of hydrogen in animal feed (dimensionless)

$\mathrm{F}_{\mathrm{Hm}}=$ fraction of hydrogen in animal product $m$ (dimensionless) and other terms are as previously defined.

The models for ${ }^{14} \mathrm{C}$ are similar to those for $3 \mathrm{H}$. The concentration of carbon-14 in vegetation from irrigation is 
TABLE 4.33. Fractions of Hydrogen and Carbon in Environmental

Media, Vegetation, and Animal Products

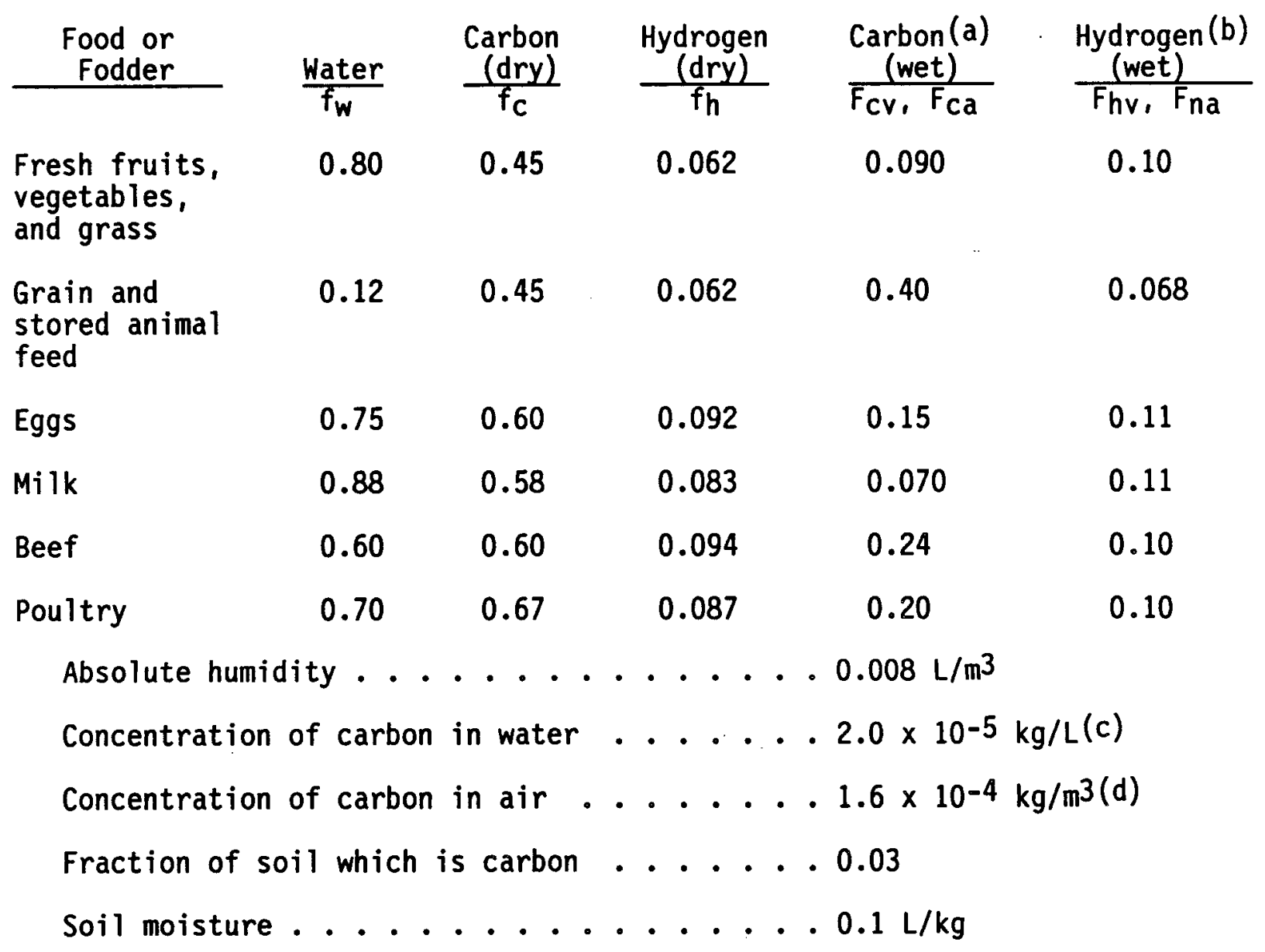
(a) $F_{C V}$ or $F_{c a}=f_{c}\left(1-f_{w}\right)$
(b) $F_{h v}$ or $F_{h a}=f_{w} / 9+f_{h}\left(1-f_{w}\right)$
(c) Assumes a typical bicarbonate concentration of $100 \mathrm{mg} / \mathrm{L}$
(d) Assumes a typical atmospheric $\mathrm{CO}_{2}$ concentration of $320 \mathrm{ppm}_{\mathrm{v}}$ 


$$
c_{C p}=C_{w C} I \text { tep } \frac{0.1}{0.01 P \lambda s C}\left[1-\exp \left(-\lambda s c t e_{p}\right)\right]
$$

where $C_{C p}=$ concentration of $14 C$ in plant type $p(C i / k g)$

$C_{W C}=$ concentration of $14 \mathrm{C}$ in irrigation water

$\lambda s C=$ effective removal rate constant for $13 C$ in soil (sec -1$)$

$0.1=$ the assumed uptake of $10 \%$ of plant carbon from soil

$0.01=$ the average fraction of soil that is carbon

and other terms are as previously defined. The concentration of $14 \mathrm{C}$ in crops from atmospheric contamination is calculated as

$$
C_{C p}=C_{a C} F_{C p} / P_{C}
$$

where $\mathrm{CaC}=$ concentration of $14 \mathrm{C}$ in air $\left(\mathrm{Ci} / \mathrm{m}^{3}\right)$

$F_{C p}=$ fraction of carbon in the $p l$ ant (dimensionless)

$P_{C}=$ concentration of carbon in $a i r\left(\mathrm{~kg} / \mathrm{m}^{3}\right)$.

The concentration of $14 \mathrm{C}$ in animal product:s is calculated as

$$
C_{C m}=\frac{C_{C p Q f}+C_{w C Q w}}{F_{C f Q f}+F_{C w Q w}} F_{C m}
$$

where $\mathrm{C}_{\mathrm{Cm}}=$ concentration of $14 \mathrm{C}$ in animal product $\mathrm{p}(\mathrm{Ci} / \mathrm{kg}$ or $\mathrm{Ci} / \mathrm{L})$

$C_{C p}=$ concentration of $14 \mathrm{C}$ in crop used for animal feed $(\mathrm{Ci} / \mathrm{kg})$

$\mathrm{CWC}_{\mathrm{C}}=$ concentration of $14 \mathrm{C}$ in animal drinking water $(\mathrm{Ci} / \mathrm{L})$

$F C f=$ fraction of carbon in animal feed (dimensionless)

$F_{C W}=$ fraction of carbon in animal drinking water (dimensionless)

$\mathrm{FCm}_{\mathrm{m}}=$ fraction of carbon in animal product $\mathrm{m}$ (dimensionless)

and other terms are as previously defined. This expression can be simplified for airborne releases by noting that the water concentration $\left(\mathrm{CWC}_{\mathrm{C}}\right.$ ) is zero, and the carbon content in plants is much higher than in water $(F C F \gg F C W$ ). The animal product concentration then becomes:

$$
C_{C m}=C_{C p} \frac{F_{C m}}{F_{C f}}
$$




\subsection{LONG-TERM CALCULATIONS}

This section describes the mathematical models and algorithms used in the DITTY program. The discussion is divided into five parts as follows:

- source terms - specification and use

- airborne release - modes for estimating average air concentrations downwind of the release, and definition of the exposed population

- waterborne release - model for definition of exposed populations

- environmental concentrations - models to estimate important environmental pathway concentrations

- pathway dose calculations - models to determine total population doses from important pathways.

Most of the descriptions parallel those in the previous five sections, 4.3 to 4.7 , modified for the 10,000 -year integral formulation.

\subsubsection{Incorporation of Long-Term Sources}

The environmental source terms represent the rate at which radionuclides enter the environment through airborne or waterborne routes. The generation of source terms is normally performed by sophisticated computer programs for ground water transport. To interface easily with these programs, a general method for source-term specification was chosen: all radionuclide releaserate data are provided as time/rate data pairs. Each data pair gives a time (years after a reference time) and a release rate in curies per year for a given radionuclide. The data for each radionuclide are provided in a set of data pairs with up to 450 time points. Such a set is provided for each radionuclide of interest. Using this procedure, a different set of time points can be used for each radionuclide. Because of the great variation in transport properties among the radionuclides of interest in radioactive waste, it is important to be able to specify releases over a range of time periods.

To use the release rate data within the 70 -year increment calculational scheme, the release rate data are interpolated and integrated to give the total activity released in each 70 -year increment. 
A special option is included to describe an acute release to the environment at the beginning of the time period. This acute release can be selected for either airborne or waterborne pathways. No more than one acute release per computer simulation is allowed.

\subsubsection{Long-Term Airborne Releases}

To estimate the radiation dose received from releases to the atmosphere, it is necessary to consider atmospheric transport of radionuclides. The atmospheric processes transport the radionuclides throughout the region surrounding the release point. The resulting distribution of material is important in determining the radiation exposure received by members of the regional population through potential exposure pathways. This section describes the methods available for specifying and estimating atmospheric dispersion.

Calculating population exposure is belsed on a spatial grid as described in Section 4.4.3. The population data set is specified as the number of people living within each area element of the grid at a given time. Sixteen directions and up to 10 distance intervals are used. The atmospheric dispersion calculation is based on joint frequency of occurrence data for wind speed, wind direction, and atmospheric stability for the site. One set of joint frequency data is given to be used with all population data. The downwind normalized air concentration is estimated for each area element using the long-term average equation provided in Section 4.3. If values of normalized air concentrations are already known, they may be supplied as input rather than calculations. The dispersion factors are used with the population distribution data to provide a population-weighted dispersion factor. The factor represents a population-weighted estimate of the average normalized air concentration for the region. The factor is calculated as follows:

$$
P M(t)=\sum_{i=1}^{\text {directions }} \sum_{j=1}^{\text {distances }} P_{i j}(t)\left(\bar{\chi} / Q^{\prime}\right)_{i j}
$$


where $P M(t)$ is the population-exposure factor at time $t$ (person-sec/m 3 ), and $P_{i j}(t)$ is the number of people living in the area interval in direction $j$ at distance $i$ at time $t$ (persons). As the equation indicates, the population exposure factor is a function of time. Changes in population over the 10,000-year period will be the primary cause of change in the populationexposure factor. While climate changes may also affect the factor, such changes are difficult to predict and are not considered.

Two options that determine population-exposure factors are available in DITTY for normalized air concentration values. When estimates of the total population change are known, the population exposure factor for the initial time may be calculated, and values for remaining time increments will then be calculated by ratio to population changes with time. If population distribution data are known as a function of time, they may be used to calculate population-exposure factors at each of the specified times, which are, in turn, interpolated for each 70-year increment.

Specifying population exposure factors can occur by direct input of factor at defined time points or by calculation from defined meteorological data and population data. When population exposure values are input, interpolations are made to determine the values at the midpoint of each 70 -year time increment.

\subsubsection{Long-Term Waterborne Releases}

Pathways associated with waterborne releases include external exposure to contaminated water and sediment; ingestion exposure from drinking water, farm products (via irrigation), and aquatic foods; and inhalation of resuspended material after irrigation. The release of activity to water is described by a release rate in curies per year defined at specific times. The activity released is assumed to result in exposure of a regional population. Specification of the number of people exposed to waterborne pathways is performed similarly to definition of population for airborne pathways. The major difference is that only the total population is specified (spatial distribution is not needed).

Two methods are available for defining population data for waterborne release. The first method is to define the population exposed during each of 
the 143 70-year periods. The second method is to give the population present at specified times for interpolation at the midpoint of each 70-year period.

\subsubsection{Long-Term Environmental Concentrations}

In estimating exposure of the regional population from airborne as well as waterborne releases, it is necessary to determine radionuclide concentrations in several environmental media and exposure-pathway media. This section describes models used to estimate air, water, soil, sediment, and food concentrations for the important pathways. A11 radionuclide concentrations are-expressed as time integrals over a 70-year period. This is done to facilitate dose calculations that use the concentration data.

Input to the pathway analysis is the total release of curies in each 70-year time period. The total release is combined with population and dispersion parameters to estimate an environmental parameter for the exposure analysis. For airborne releases, the value is calculated as:

$$
A_{c}(i, t)=P M(t) \frac{3.16 \cdot 10^{-8}}{\lambda_{r i}} \int_{t_{0}}^{t_{0}+70} a_{i}(t) d t
$$

where $A_{C}(i, t)=$ population-weighted airborne release for radionuclide $i$ over 70 years (person-Ci-yr2/m3)

$\operatorname{PM}(t)=$ population exposure factor for time period $t$ (person-sec/m3)

$\lambda_{r i}=$ radiological decay constant for radionuclide $i(y r-1)$

$a_{j}(t)=$ release rate of radionuclide $i$ at time $t$ ( $\mathrm{C} / \mathrm{yr}$ )

$3.169 \cdot 10-8=$ conversion factor $(\mathrm{yr} / \mathrm{sec})$.

For waterborne releases, the water concentration parameter is calculated as:

$$
W_{c}(i, t)=\frac{P(t) N M 1.119 \cdot 10^{-9}}{F_{r} \lambda_{r i}} \int_{t_{0}}^{t_{0}+70} c(t) d t
$$

where $W_{C}(i, t)=$ population-weighted water concentration for radionuclide $i$ and time period $t$ (person-ci-yr2/L) $P(t)=$ population exposed to water for time period $t$ (persons) 


$$
\begin{aligned}
N & =\text { reconcentration factor (dimensionless) } \\
M & =\text { mixing ratio (dimensionless) } \\
F_{r} & =\text { flow rate of receiving water }\left(\mathrm{ft}^{3} / \mathrm{sec}\right) \\
C(t) & =\text { release rate to the receiving water }(\mathrm{Ci} / \mathrm{yr}, \text { at time } \mathrm{t} \text { ) } \\
1.119 \cdot 10^{-9} & =\text { conversion factor, } \frac{1}{28.31 \frac{\mathrm{L}}{\mathrm{ft}^{3}} 3.156 \cdot 10^{7} \mathrm{yr} \frac{\mathrm{sec}}{\mathrm{yr}}}\left(\frac{\mathrm{ft}^{3} \mathrm{yr}}{\mathrm{L} \mathrm{sec}}\right) .
\end{aligned}
$$

The airborne pathway analysis used the air concentration parameter, $A_{C}(i, t)$, to determine several environmental concentrations:

- $S_{c a}(i, t)$, soil concentration parameter for radionuclide $i$, person-Ci-yr $2 / k g$

- $L_{c a}(i, p, t)$, time integral of leaf concentration for radionuclide $i$ and pathway $p$, from air deposition and resuspension over the current period $t$, person- $\mathrm{C} i-\mathrm{yr}^{2} / \mathrm{kg}$

- $P_{c a}(i, p, t)$, time integral of plant concentration for radionuclide $i$ and pathway $p$, from air-deposited contaminants on plants and root uptake over the current period $t$, person-Ci-yr2/kg .

The soil concentration parameter is calculated from the air concentration parameter assuming deposition to be at a uniform rate over the 70-year period:

$$
S_{c a}(i, t)=\frac{A_{c}(i, t) V_{d i}}{7.069 \cdot 10^{9}}\left(\frac{1-e^{-\lambda_{b} t}}{\lambda_{b}}\right)
$$

where $\quad V_{d i}=$ deposition velocity for radionuclide $i(\mathrm{~m} / \mathrm{sec})$

$$
\lambda_{b}=\text { environmental decay constant }(y r-1)
$$

$7.069 \cdot 10^{9}=$ constant $\left(224 \mathrm{~kg} / \mathrm{m}^{2} \cdot 3.156 \cdot 107 \mathrm{sec} / \mathrm{yr}\right)$.

The environmental decay constant, $\lambda_{b}$, is calculated as the sum of a radiological decay constant, $\lambda_{r i}$, and a soil removal constant for weathering $\lambda_{w i}$. The soil removal constant is a correction for long-term leaching of deposited radionuclides out of the soil rooting and resuspension zones. The values given for $\lambda_{w i}$ are calculated using the formula of Baes and Sharp (1981) as given in Equation (4.6.3). 
The time integral of leaf concentration is calculated from the air and soil concentration parameters assuming a constant soil concentration equal to the value at the end of the 70-year period. This is a conservative assumption. The equation is:

$$
\begin{aligned}
L_{c a}(i, p, t)= & \frac{r V_{d i}}{Y_{p}}\left[A_{c}(i, t)+1.49 \cdot 10^{-8} S_{c a}(i, t)\right] \\
& \left(\frac{1-e^{-\lambda} i^{\top} / 365}{\lambda_{e i}}\right) 3.156 \cdot 10^{7}
\end{aligned}
$$

where $\quad Y_{p}=$ crop yield for food pathway $p\left(\mathrm{~kg} / \mathrm{m}^{2}\right)$

$\lambda_{e i}=$ effective retention rate constant for radionuclide $i(y r-1)$

$r=$ interception fraction, discussed in Section 4.7.4

$\lambda_{e j}=\lambda_{r i}+18.0838(y r-1)$

18.0838 = rate constant for a 14 -day half time

$T_{p}=$ growing period for food pathway, $p$ (days)

0.25 = interception fraction (dimensionless)

$1.49 \cdot 10-8=$ conversion factor, $1 \cdot 10-9(\mathrm{~m}-1) \cdot 224 / 15\left(\mathrm{~kg} / \mathrm{m}^{2}\right)$

$365=$ days per year

$3.156 \cdot 107=$ seconds per year.

The factor of $1 \cdot 10^{-9}\left(\mathrm{~m}^{-1}\right)$ represents a resuspension factor assumed constant, characteristic of aged deposition material (Anspaugh et al. 1975). It is assumed that the resuspended activity deposits on the plants near the soil from which it was suspended. Downwind transport of resuspended activity is not considered. The factor of 224 is the soil area density to a depth of $15 \mathrm{~cm}$ of $224 \mathrm{~kg} / \mathrm{m}^{2}$, and $15 \mathrm{~cm}$ is the plow depth through which the contamination is distributed. This value is included so that only the top centimeter of material (1/15 of the total) is considered available for resuspension. The leaf concentration as calculated above represents the time integral over a 70-year period.

The radionuclide concentration in edible parts of the plant includes material from direct deposition plus material from root uptake: 


$$
P_{c a}(i, p, t)=L_{c a}(i, p, t) T_{v p}+S_{c a}(i, t) B_{i v}
$$

where $P_{c a}(i, p, t)=$ time integral of plant concentrations for plant type $p$ for radionuclide $i$ and time period $t$ (person- $\mathrm{C}-\mathrm{yr} \mathbf{2} / \mathrm{kg}$ )

$T_{v p}=$ translocation factor of externally deposited radionuclides to edible parts of the plant (dimensionless)

$B_{i v}=$ concentration ratio for plant uptake of radionuclide $i$, $\mathrm{Ci} / \mathrm{kg}$ (wet weight plant) per $\mathrm{Ci} / \mathrm{kg}$ (dry weight soil)

and other terms are as previously defined.

The concentration used for calculating uptake by population is the plant concentration for plant pathways. For animal pathways, the uptake is calculated as:

$$
A_{c a}(i, p, t)=P_{c a}(i, p, t) \text { Sip } Q_{p}
$$

where $A_{C a}(i, p, t)=$ time integral of animal product concentration for radionuclide $i$, animal product $p$, and time period $t$, period-Ci-yr2/kg (person-Ci/yr $2 / L$ for milk)

$S_{i p}=$ transfer coefficient of radionuclide $i$ from daily intake by animal to edible portion of animal product, $\mathrm{Ci} / \mathrm{L}$ (milk) per $\mathrm{Ci} /$ day or $\mathrm{Ci} / \mathrm{kg}$ (animal product) per Ci/day

$Q_{p}=$ the consumption rate of contaminated feed or forage by the animal for animal product $\mathrm{p}$ ( $\mathrm{kg} / \mathrm{day})$.

The waterborne pathway analysis uses the water concentration $W_{C}(i, t)$ to determine the following environmental concentrations:

- $S_{C W}(i, t)$, soil concentration for radionuclide $i$ for the current period $t$ for irrigation deposition, person-Ci-yr $2 / \mathrm{kg}$

- $S_{d w}(i, t)$, sediment deposition concentration for radionuclide $i$ for current period $t$ for shoreline of contaminated water body, person-Ci-yr $2 / \mathrm{m}^{2}$ 
- $L_{c w}(i, p, t)$, leaf concentrations for radionuclide $i$, plant type $p$, and time period $t$ for irrigation deposition and resuspension, person-Ci-yr $2 / \mathrm{kg}$

- $P_{c w}(i, p, t), p l a n t$ concentration of radionuclide $i$ and pathway $p$, from irrigation deposition onto plant and root uptake through soil, person-Ci-yr $2 / \mathrm{kg}$.

The soil concentration at the end of the period is calculated assuming uniform deposition over the period.

$$
S_{c W}(i, t)=\frac{W_{c}(i, t) I T_{i}}{224}\left(\frac{1-e^{-\lambda_{b} T}}{\lambda_{b}}\right)
$$

where $\quad I=$ irrigation rate $\left(L / \mathrm{m}^{2} \mathrm{mo}\right)$

$$
\begin{aligned}
T_{j} & =\text { irrigation period }(\mathrm{mo} / \mathrm{yr}) \\
224 & =\text { soil area density }\left(\mathrm{kg} / \mathrm{m}^{2}\right) .
\end{aligned}
$$

The exponential term represents the integral over the 70 -year period.

The concentration in the sediment is calculated similarly as:

$$
S_{d w}(i, t)=W_{c}(i, t) 25300
$$

where 25300 is the constant to represent deposition to sediment, $L / \mathrm{m}^{2} / \mathrm{yr}$. The value of the sediment deposition constant (25300) is the same as that described in Section 4.7.7 with the units converted.

The concentration on leaves is calculated for contributions from direct irrigation deposition plus resuspension from soil:

$$
\begin{gathered}
L_{c W}(i, p, t)=\frac{0.25}{Y_{p}}\left[W_{c}(i, t) \cdot I \cdot 12+s_{c W}(i, t) \cdot 0.47 V_{d i}\right] \\
\left(\frac{1-e^{-\lambda} e^{T} T_{p}^{/ 36 i 5}}{\lambda_{e i}}\right)
\end{gathered}
$$


where $0.25=$ plant interception fraction for irrigation (dimensionless)

$Y_{p}=p l a n t$ yield for $p l a n t$ type $p\left(\mathrm{~kg} / \mathrm{m}^{2}\right)$

$12=$ unit conversion constant (mo/yr)

$0.47=$ conversion factor for resuspension

$0.47=\left(10^{-9} \mathrm{~m}^{-1}\right)\left(3.156 \bullet 10^{7} \mathrm{sec} / \mathrm{yr}\right)\left(224 \mathrm{~kg} / \mathrm{m}^{2}\right)(1 / 15)$

$V_{d i}=$ deposition velocity of resuspended activity $(\mathrm{m} / \mathrm{sec})$

$\lambda_{e i}=$ environmental weathering constant for 14-day half time, $y r-1$

$\lambda_{e i}=\lambda_{r i}+18.0838$.

The radionuclide concentration in edible parts of the plant is calculated for root uptake plus direct deposition as

$$
P_{c w}(i, p, t)=L_{c w}(i, p, t) T_{v p}+S_{c w}(i, t) B_{i v}
$$

where $T_{v p}=$ translocation factor of externally deposited radionuclides to edible parts of plant (dimensionless)

$B_{i v}=$ concentration ratio for plant uptake of radionuclide $\mathrm{i}, \mathrm{Ci} / \mathrm{kg}$ (wet weight plant) per $\mathrm{Ci} / \mathrm{kg}$ (dry weight soil).

The total uptake of plants during the 70 -year period is given by the above plant concentration parameter. For animal products, uptake is calculated for animal consumption of plants plus animal consumption of water as

$$
A_{c w}(i, p, t)=S_{i p}\left[P_{c w}(i, p, t) Q_{p}+W_{c}(i, t) Q_{p w}\right]
$$

where $A_{C W}(i, p, t)=$ time integral of animal product $p$, concentration in time $t$, for radionuclide $i$ from waterborne pathways, person$\mathrm{Ci}-\mathrm{yr} \mathrm{r}^{2} / \mathrm{kg}$ (person-Ci-yr $2 / \mathrm{L}$ for milk)

$S_{i p}=$ transfer coefficient of radionuclide $i$ from daily intake by animal to edible portion of animal product $\mathrm{p}, \mathrm{Ci} / \mathrm{L}$ (milk) per Ci/day or $\mathrm{Ci} / \mathrm{kg}$ (animal product) per $\mathrm{Ci} /$ day

$Q_{p}=$ the consumption rate of contaminated feed or forage by animal for animal type $p(\mathrm{~kg} /$ day)

$Q_{p w}=$ the consumption rate of water by animal for animal type $p(L / d)$. 
The time-integrated concentration in aquatic foods is calculated from the water concentrations as

$$
A_{f w}(i, p, t)=W_{c}(i, t) B_{i p}
$$

where $A_{f w}(i, p, t)=$ time-integrated concentration of radionuclide $i$ in aquatic food $p$ (person-Ci-yr $2 / \mathrm{kg}$ )

$B_{i p}=$ bioaccumulation factor for radionuclide $i$ and aquatic food $\mathrm{p}(\mathrm{Ci} / \mathrm{kg}$ per $\mathrm{Ci} / \mathrm{L})$.

The time-integrated water concentration for the drinking water pathway is calculated as

$$
A_{d w}(i, t)=W_{C}(i, t) C_{i}
$$

where $A_{d w}(i, t)=$ time-integrated concentration in drinking water for radionuclide $i$ and time period $t$ (person-Ci-yr2/L) $C_{j}=$ water purification plant cleanup factor (dimensionless).

The radionuclides tritium and carbon-14 are handled in the special manner described in Section 4.7.9.

\subsubsection{Long-Term Pathway Dose Calculations}

The total dose received by the regional population is estimated as the sum of contributions from all pathways. The doses are calculated as the population dose received in each 70 -year time period from material released to the environment during that period plus all previous periods. Precalculated dose conversion factors are used to estimate dose from uptake and environmental concentration. The exposure pathways are described in the following sections.

\section{Air Submersion}

Contributions for external exposure from air submersion are included for 1) submersion in the release plume, 2) submersion in resuspended activity from an initial airborne release, and 3) submersion in suspended activity from an initial irrigation water deposition. The dose is calculated as 


$$
\begin{aligned}
D_{o e i}= & D_{e i} \lambda_{r i} 3.156 \cdot 10^{7}\left[A_{c}(i, t)+1.49 \cdot 10^{-8}\right. \\
& \left.\cdot\left(S_{c a}(i, t)+S_{c w}(i, t)\right)\right]
\end{aligned}
$$

where $D_{o e i}=$ population dose from air submersion to organ 0 from radionuclide $i$ (person-rem)

Dei = external exposure dose conversion factor for air submersion (rem per $\mathrm{Ci}-\mathrm{sec} / \mathrm{m}^{3}$ )

$\lambda_{r i}=$ radiological decay constant for radionuclide $i(y r-1)$

$3.156 \cdot 107=$ conversion factor $(\mathrm{sec} / \mathrm{yr})$

$A_{C}(i, t)=$ time-integrated air concentration of radionuclide $i$ in time period $t$ (person-Ci-yr $2 / \mathrm{m}^{3}$ )

$1.49 \cdot 10-8=$ resuspension factor constant $\left(\mathrm{kg} / \mathrm{m}^{3}\right)$

$=\left(1 \cdot 10^{-9} \mathrm{~m}^{-1}\right)\left(224 \mathrm{~kg}\right.$ soi $\left.1 / \mathrm{m}^{2}\right)(1 / 15)$

$S_{c a}(i, t)=$ soil concentration parameter from airborne release of radionuclide $i$ for time period $t$ (person- $\mathrm{C} i-\mathrm{yr}^{2} / \mathrm{kg}$ )

$S_{\mathrm{CW}}(i, t)=$ soil concentration parameter from waterborne release of radionuclide $i$ for time period $t$ (person- $\mathrm{C} i-\mathrm{yr}^{2} / \mathrm{kg}$ ).

The contribution from deposited material is based on the concentration at the end of the current period. This concentration is assumed to occur throughout the period, which is a conservative assumption by no more than a factor of two. Resuspension is based on a constant resuspension factor of $10^{-9} \mathrm{~m}^{-1}$, representing resuspension of aged deposited material (Anspaugh et al. 1975). The resuspended activity is assumed to expose individuals in the vicinity of the soil from which it was suspended. Downwind transport of resuspended activity is not considered. The decay constant, $\lambda r_{j}$, is included to convert the units of radionuclide concentration from mass to activity. The calculations are performed in units proportional to mass to meet requirements of the chain decay processor, BCHAIN.

\section{Inhalation}

Inhalation exposure includes contributions from the released airborne activity plus the resuspended activity from airborne and irrigation water deposition. The dose is calculated as: 


$$
\begin{aligned}
D_{\text {ohi }}= & D_{\text {hio }} 3.156 \cdot 10^{7} \lambda_{\text {ri }}\left[A_{c}(i, t)+1.49 \cdot 10^{-8}\right. \\
& \left.\left(S_{c a}(i, t)+S_{c W}(i, t)\right)\right]
\end{aligned}
$$

where $D_{\text {ohi }}=$ dose from inhalation exposure from organ 0 from radionuclide $i$ (person-rem)

Dhio = inhalation dose conversion factor for radionuclide $i$ and organ o (rem per $\mathrm{Ci}-\mathrm{sec} / \mathrm{m}^{3}$ )

and other terms are as previously defined.

Terrestrial Ingestion Pathways

Terrestrial pathways include ingestion of crops and animal products. The seven terrestrial ingestion pathways available are vegetable, grain, eggs, milk, beef, pork, and poultry. The close for each pathway is calculated from the time-integrated food-product concentration. For plants, the dose is calculated as

$$
D_{o t i}=D_{g i o} \lambda_{r i} U_{p}\left[p_{c a}(i, p, t)+P_{c w}(i, p, t)\right]
$$

and for animal products, the dose is calculated as

$$
D_{\text {oti }}=D_{\text {gio }} \lambda_{r i} U_{p}\left[A_{c a}(i, p, t)+A_{c w}(i, p, t)\right]
$$

where $D_{o t} i=$ dose from terrestrial ingestion pathways for organ 0 (person-rem)

Dgio $=$ ingestion dose conversion factor for radionuclide $\mathfrak{i}$ and organ o (rem/Ci)

$U_{p}=$ the usage rate by humans of food product $p(\mathrm{~kg} / \mathrm{yr}$ or $\mathrm{L} / \mathrm{yr}$ for milk)

$P_{c a}(i, p, t)=$ time integral of plant concentration from airborne pathways for radionuclide $i$, plant type $p$, and time period $t$ (person-Ci-yr $2 / \mathrm{kg}$ ) 
$P_{C W}(i, p, t)=$ time integral of plant concentration from waterborne pathways for radionuclide $i$, plant type $p$, and time period $t$ (person-Ci-yr $2 / \mathrm{kg}$ )

$A_{c a}(i, p, t)=$ time integral of animal product concentration from airborne pathways for radionuclide $i$, animal product $p$, and time period $t$ (person-Ci-yr $2 / \mathrm{kg}$ or person-Ci-yr $2 / \mathrm{L}$ for $\mathrm{milk}$ )

$A_{C W}(i, p, t)=$ time integral of animal product concentration from waterborne pathways for radionuclide $i$, animal product $p$, and time period $t$ (person-Ci-yr $2 / \mathrm{kg}$ or person-Ci-yr $2 / L$ for milk).

The total dose from terrestrial ingestion pathways is calculated by summing contributions from all plant and animal product food types.

\section{Aquatic Ingestion Pathways}

Ingestion pathways resulting from release of radionuclide to surface or ground water include ingestion of drinking water and aquatic foods. The five pathways available are fish, crustacea, molluscs, water plants, and drinking. water. The dose for each pathway is calculated from the time-integrated aquatic media concentration as follows for aquatic foods:

$$
D_{\text {oai }}=D_{g i o} \lambda_{r i} U_{a} A_{f w}(i, p, t)
$$

and for drinking water:

$$
D_{\text {oai }}=D_{g i o} \lambda_{r i} U_{a} A_{d w}(i, t)
$$

where $D_{o a i}=$ dose from ingestion of aquatic food or water for organ 0 (person-rem)

$U_{a}=$ the usage rate by humans of aquatic-food pathway a $(\mathrm{kg} / \mathrm{yr}$ or L/yr for drinking water)

$A_{f w}(i, p, t)=$ time integral of aquatic food $p$, concentration for radionuclide $i$, in time period $t$ (person-Ci-yr $2 / \mathrm{kg}$ )

$A_{d w}(i, t)=$ time integral of drinking water concentration for radionuclide $i$ in time period $t$ (person-Ci-yr2/L). 


\section{External Exposures}

External exposures result from proximity to contaminated ground, shoreline, and water. Swimming and shoreline doses are calculated from the timeintegrated sediment concentration and water concentration as

$$
D_{\text {oew }}=\lambda_{r i}\left[S_{d w}(i, t) D_{o s} U_{s h} w+w_{c}(i, t) D_{o w} U_{s w}\right]
$$

where $D_{\text {oew }}=$ dose from external exposure to shoreline and water for organ o (person-rem)

$D_{O S}=$ external dose factor for organ o for exposure to contaminated soil or shoreline (rem/h per $\mathrm{Ci} / \mathrm{m}_{2}$ )

$D_{\text {ow }}=$ external dose factor for organ o for submersion in contaminated water ( $\mathrm{rem} / \mathrm{h}$ per $\mathrm{Ci} / \mathrm{L}$ )

$U_{s h}=$ time of exposure to contaminated shoreline ( $\left.h / y r\right)$

$U_{S W}=$ time of exposure to contaminated water $(h / y r)$

$S_{d w}(i, t)=$ sediment concentration parameter for radionuclide $i$ for the current time period $t$ (person-Ci-yr/m²)

$W_{C}(i, t)=$ time integral of water concentration for radionuclide $i$ and time period $t$ (person-Ci-yr2/L)

$W=$ shore-width factor for shoreline exposure (dimensionless).

The shore-width factor is an approximate correction to the infinite-plane geometry of the external exposure factors. To correct for the actual geometry of a river bank or beach, a shore-width factor is applied corresponding to the particular exposure situation. Suggested shore-width factors are given in Table 4.31. Contaminated soil call result from deposition of airborne material or from irrigation with contaminated water. The dose from external exposure to contaminated soil is calculated as

$$
D_{\text {oes }}=\lambda_{r i} E_{t} D_{\text {ow }} 224\left[S_{c a}(i, t)+S_{c w}(i, t)\right]
$$

where $D_{\text {oes }}=$ dose from external exposure t:o soil for organ o (person-rem)

$E_{t}=$ time of exposure to contaminated ground $(h / y r)$

$S_{c a}(i, t)=$ soil concentration due to airborne deposition of radionuclide $i$ at the end of time period $t$ (person-Ci-yr/ $\mathrm{kg}$ ). 


$$
S_{\mathrm{CW}}(i, t)=\text { soil concentration due to waterborne deposition of radionuclide }
$$
$i$ at the end of time period $t$ (person-Ci-yr/kg)

$$
224=\text { soil areal density }\left(\mathrm{kg} / \mathrm{m}_{2}\right) \text {. }
$$

and other terms are as previously defined. Note that the external exposure is based on the integrated soil concentration for the current 70 -year period. 


\subsection{SOLUTION TECHNIQUES}

The GENII package of computer codes is written in standard FORTRAN 77 using a Leahey compiler. Most routines are simple algebraic solutions, and no special logic is employed. The exception is the INTDF code, which calculates internal dose factors and uses a general purpose solver for ordinary differential equations. The solver used is from the ODEPACK collection developed at Lawrence Livermore National Laboratory (Hindmarsh 1983). The specific portion if ODEPACK used is called LSODES.

The LSODES package solves explicit systems of stiff differential equations. Stiffness is related to the rate constants of the equations and may require large numbers of iterations for some solution techniques. LSODES uses a backwards differentiation formulation, based on the multistep methods first implemented by C. W. Gear (1971). The following description was adapted from a publication by Hindmarsh (1.983):

When solving a stiff system of size $N$, of the general form

$$
\dot{y} \equiv d y / d t=f(t, y)
$$

the LSODES package makes use of the Jlacobian matrix of partial derivates

$$
J=\partial f / \partial y
$$

For stiff problems, LSODES uses the Backward Difference Formula (BDF)

$$
\begin{aligned}
y n & =\sum_{i=1}^{q} a_{i} y_{n-1}+h \beta_{0} \dot{y}_{n} \\
& =a_{n}+h \beta_{0} f\left(t_{n}, y_{n}\right)
\end{aligned}
$$

where again $q$ is the order (here $1 \leq q \leq 5$ ), and $\beta_{0}>0$. Stiffness makes functional iteration fail to converge for the step sizes of 
interest, because of strong dependencies in $f$ upon $y$. Therefore, LSODES uses a modified Newton iteration

$$
-P\left[y_{n}(m+1)-y_{n}(m)\right]=y_{n}(m)-a_{n}-h \beta_{0} f\left(t_{n}, y_{n}(m)\right)
$$

where $P$ is an NxN matrix approximating the Jacobian of the algebraic system to be solved:

$$
P \simeq I-h \beta_{0} J, J=\partial f / \partial y
$$

(Here I denotes the $N \times N$ identity matrix.) Again a prediction $y_{n}(0)$ is formed from an analogous explicit formula. This iteration differs from a true Newton method in that $J$ is only evaluated periodically. In fact, $\mathrm{J}$ is evaluated only at predicted values $y_{n}(0)$ and only on those steps where a new value appears necessary, on the basis of a convergence failure or other indication. The same value of $P$ (or its LU decomposition, if used) is used over all iterations in any one step, and typically also over several time steps, until a reevaluation of $J$ and $P$ is called for. (In the case of the LSODES solver, $P$ is sometimes updated and LU-decomposed without a reevaluation of $\mathrm{J}$. ) Again, $h$ and $q$ are both varied to meet local error tolerance requirements.

In applying the BDF method to large stiff problems, it is important to note that a numerical solution of the linear system

$$
\begin{aligned}
& P x=r \\
& (x=\text { correction vector, } r=\text { residual vector })
\end{aligned}
$$

can very often easily take advantage of a sparse structure in $P$. This is accomplished either through suitable structured LU decompositions, or through iterative linear system methods that use a given matrix structure. The use of structure is especially important in solving ODE systems that come from time-dependent partial differential equation (PDE) systems by the method of lines, whereby spatial variables are discretized, leaving ODE's in time.

The LSODES package solves explicit systems $\dot{y}=f$, but treats the Jacobian matrix $J$ as a general sparse matrix in the stiff case. In LSODES, linear systems are solved using parts of the Yale Sparse Matrix Package (YSMP) (Eisenstat et al. 1977a and 1977b). Recall that the systems to be solved have the form

$$
P x=r, P=I-h \beta_{0} J
$$

where $x$ is a correction vector, $h$ is the step size, and $\beta_{0}$ is a scalar depending on the current method order. The solution of these systems involves several phases:

(a) Determination of sparsity structure. This is inferred from calls to the $f$ routine. 
(b) Determination of pivot order. Diagonal pivot locations are chosen, and the choice is based on maintaining maximum sparsity. This is done by YSMP (ODRV module) (Eisenstat et al. 1977a). The ordering algorithm (minimum degree algorithm) operates only on a symmetric sparsity structure, and in LSODES the structure used for this is that of $\mathrm{J}+\mathrm{JT}$.

(c) Symbolic LU factorization of the matrix P. This is based only on sparsity and the pivot order, and uses the module in YSMP designed for nonsymmetric matrices with compressed pointer storage (CDRV module) (Eisenstat et al. 1977b).

(d) Construction of $\mathrm{J}$. This is done internally by difference quotients. The number of $f$ evaluations needed is kept to a minimum by a column grouping technique due to Curtis, Powell, and Reid (1974). J is stored internally in an appropriate packed form. Evaluations of $\mathrm{J}$ are done only occasionally, as explained below.

(e) Construction of $P=I-h \beta_{0} J$. LSODES does not force a reevaluation of $J$ whenever the existing $P$ is deemed unsuitable for the corrector iterations. Instead, when the value of $\mathrm{J}$ contained in the stored value of $P$ is likely to be usable (and $P$ is not, only because $h \beta_{0}$ has changed significantly), then a new matrix $P$ is constructed from the old one, with careful attention to roundoff error. This cuts down greatly on the total number of $\mathrm{J}$ evaluations.

(f) Numerical LU factorization of $P$. This is done by YSMP (CDRV module) in sparse form, and the array containing $P$ is saved in the process. Because of the absence of partial pivoting for numerical stability, this operation can conceivably fail. However, this has only rarely been observed in practice, and if it does occur (with a current value of $J$ ), the step size $h$ gets reduced and the difficulty disappears.

(g) Solution of $P x=r$. This is done by YSMP (CDRV module) using the existing sparse factorization of $P$. Because a modified Newton iteration is used, many values of $r$ (i.e., many linear systems) can arise for the same $P$, and the separation of the various phases takes advantage of that: fact.

The first three phases and part of the fourth (column grouping for difference quotients) are normally done only at the start of the problem. However, the user can specify that the sparsity structure is to be redetermined in the middle of the problem, and then these operations are repeated. 



\subsection{REFERENCES}

American Society of Mechanical Engineers (ASME). 1986. Quality Assurance Program Requirements for Nuclear Facilities. ANSI/ASME NQA-1, American National Standards Institute, New York, New York.

Anspaugh, L. R., J. H. Shinn, P. L. Phelps, and N. C. Kennedy. 1975. "Resuspension and Redistribution of Plutonium in Soils." Health Phys. $29: 571-582$.

Baes, C. F., and R. D. Sharp. 1981. Predicting Radionuclide Leaching From Root Zone Soil From Assessment Applications. CONF-81606, Oak Ridge National Laboratory, Oak Ridge, Tennessee.

Baker, D. A., G. R. Hoenes, and J. K. Soldat. 1976. F00D - An Interactive Code to Calculate Internal Radiation Doses from Contaminated Food Products. BNWL-SA-5523, Pacific Northwest Laboratory, Richland, Washington.

Bateman, H. 1910. "Solution of a System of Differential Equations Occurring in the Theory of Radio-active Transformations." Proc. Cambridge Phil. Soc. $15: 423-427$.

Blizard, E. P., ed. 1962. "Shielding." Reactor Handbook, Vol. III. Interscience Publishers, New York, New York.

Briggs, G. A. 1984. "Plume Rise and Buoyancy Effects." Atmospheric Science and Power Production, pp. 327-366. DE84005177 (DOE/TIC-27601), U.S. Department of Energy, Washington, D.C.

Brown, K. M. 1973. "Computer Oriented Algorithms for Solving Systems of Simultaneous Nonlinear Algebraic Equations." In Numerical Solution of Systems of Nonlinear Algebraic Equations. Academic Press, Inc., New York, New York.

Brown, K. M., and S. Lin. 1973. A FORTRAN Package for Multiple Linear Regression. University of Minnesota, Minneapolis, Minnesota.

Chamberlain, A. C. 1967. "Transport of Lycopodium Spores and 0ther Small Particles to Rough Surfaces." Proc. Roy. Soc. London. A296:45-70.

Codell, R. B., K. T. Key, and G. Whelan. 1982. A Collection of Mathematical Models for Dispersion in Surface Water and Ground Water. NUREG-0868, U.S. Nuclear Regulatory Commission, Washington, D.C.

Csanady, G. T. 1973. Turbulent Diffusion in the Environment. D. Reidel, Boston, Massachusetts.

Curtis, A. R., M. J. D. Powell, and J. K. Reid. 1974. "On the Estimation of Sparse Jacobian Matrices." J. Inst. Math. Applic. 13:117-119. 
Dillman, L. T. 1974. "Absorbed Gamma Dose Rate for Immersion in a SemiInfinite Radioactive Cloud." Health Phys. 27:571.

Dunster, H. J., Chaiman. 1971. Handbook of Radiological Protection Part 1: Data. SNB 11-360079-8. Radioactivity Advisory Committee, Department of Employment, Department of Health and Social Security, Ministry of Health and Social Services, Northern Ireland. Her Majesty's Stationary Office, London, England.

Eisenstat, S. C., M. C. Gursky, M. H. Schultz, and A. H. Sherman. 1977a. Yale Sparse Matrix Package: I. The Symmetric Codes. Research Report No. 112, Department of Computer Sciences, Yale University, New Haven, Connecticut.

Eisenstat, S. C., M. C. Gursky, M. H. Schultz, and A. H. Sherman. 1977b. Yale Sparse Matrix Package: II. The Nonsymmetric Codes. Research Report No. 114, Department of Computer Sciences, Yale University, New Haven, Connecticut.

Engel, R. L., J. Greenborg, and M. M. Hendrickson. 1966. ISOSHLD - A Computer Code for General Purpose Isotope Shielding Analysis. BNWL-236, Pacific Northwest Laboratory, Richland, Washington.

Etherington, H., ed. 1958. Nuclear Engineering Handbook, 1st ed. McGrawHill Book Company, New York, New York.

Eve, I. S. 1966. "A Review of the Physiology of the Gastro-Intestinal Tract in Relation to Radiation Doses from Radioactive Materials." Health Phys. $12: 131$.

Gear, C. W. 1971. Numerical Initial Value Problems in Ordinary Differential Equations, pp. 158-166. Prentice-Hall, Englewood Cliffs, New Jersey.

Gifford, F. A. 1961. "Use of Routine Meteorological Observations for Estimating Atmospheric Dispersion." Nucl. Safety. 2:47-51.

Gifford, F. A. 1968. "An Outline of Theories of Diffusion in the Lower Layers of the Atmosphere." Meteorological and Atomic Energy - 1968, pp. 65-116, TID-24190, U.S. Atomic Energy Commission, National Technical Information Service, Springfield, Virginia.

Gifford, F. A. 1976. "Turbulent Diffusing Typing Schemes - A Review." Nucl. Safety. 17:68-86.

Golder, D. 1972. "Relations Among Stability Parameters in the Surface Layer." Boundary Layer Meteorol. 3:47-58.

Goldstein, H. 1959. Fundamental Aspects of Reactor Shielding. AddisonWesley Publishing Co., Inc., Reading, Massachusetts. 
Govaerts, P., I. Mertens, and J. G. Kretz:schmer. 1983. Application of the DOSDIM-Model to Assess Doses Due to Deposited Materials Subsequent to an Accidental Atmospheric Release. Studiec:entrum voor Kernenergie, Mol, Belgium.

Hindmarsh, A. C. 1983. "Toward A Systemized Collection of ODE Solvers." Scientific Computing, R. S. Stepleman, ed. 10th IMACS World Congress on Systems Simulation and Scientific Computation, North-Holland Publishing Company, Amsterdam, The Netherlands.

Hosker, R. P. 1984. "Flow and Diffusion Near Obstacles." Atmospheric Science and Power Production, pp. 241-326. DE84005277 (D0E/TIC-27601), U.S. Department of Energy, Washington, [).C.

Houston, J. R., D. L. Strenge, and E. C. Watson. 1974. DACRIN: A Computer Program for Calculating Organ Dose from Acute or Chronic Radionuclide Inhalation. BNWL-B-389, Pacific Northwe:st Laboratory, Richland, Washington.

International Commission on Radiological Protection (ICRP). 1966. "Deposition and Retention Models for Internal Dosimetry of the Human Respiratory Tract." Health Phys. 12:173.

International Commission on Radiological Frotection (ICRP). 1972. Metabolism of Compounds of Plutonium and Other Actinides. ICRP Publication 19, Pergamon Press, New York, New York.

International Commission on Radiological F'rotection (ICRP). 1973. Alkaline Earth Metabolism in Adult Man. ICRP Publication 20, Pergamon Press, New York, New York.

International Commission on Radiological Frotection (ICRP). 1975. Report of the Task Group on Reference Man. ICRP Fublication 23, Pergamon Press, oxford, Great Britain.

International Commission on Radiological Frotection (ICRP). 1977. Recommendations of the International Commission on Radiological Protection. ICRP Publication 26, Pergamon Press, New York, New York.

International Commission on Radiological Protection (ICRP). 1979-1982. Limits for Intakes of Radionuclides by Workers. ICRP Publication 30, Pergamon Press, New York, New York: Part 1 - Annals of the ICRP Vol. 2 No. 3/4 (1979a)

Supplement to Part 1 - Annals of the ICRP Vol. 3, No. 1-4 (1979)

Part 2 - Annals of the ICRP Vol. 4 No. 3/4 (1980)

Supplement to Part 2 - Annals of the ICRP Vol. 5 (1981)

Part 3 Including Addendum to Parts 1 and 2 - Annals of the ICRP Vol. 6 No. $2 / 3(1981)$

Supplement A to Part 3 - Annals of the ICRP Vol. 7 No. 1-3 (1982)

Supplement B to Part 3 Including Addendum to the Supplements of Parts 1 and 2 - Annals of the ICRP Vol. 8, No. 1-3 (1982)

Index to ICRP Pubilcation No. 30 - Annals of the ICRP Vol. 8, No. 4 (1982) 
International Commission on Radiological Protection (ICRP). 1979b. Radionuclide Release into the Environment Assessment of Doses to Man. ICRP Publication 29, Pergamon Press, New York, New York.

International Commission on Radiological Protection (ICRP). 1986. The Metabolism of Plutonium and Related Elements. ICRP Publication 48, Pergamon Press, New York, New York.

Jaquish, R. E. 1987. Environmental Monitoring at Hanford for 1986. PNL-6120, Pacific Northwest Laboratory, Richland, Washington.

Jaquish, R. E., and B. A. Napier. 1987. A Comparison of Environmental Radionuclide Concentrations Calculated by a Mathematical Model with Measured Concentrations. PNL-SA-14720, Pacific Northwest Laboratory, Richland, Washington.

Johnson, J. R. 1982. "Fetal Thyroid Dose from Intakes of Radioiodine by the Mother." Health Phys. 43(4):573-582.

Johnson, J. R., and M. B. Carver. 1981. "A General Model for Use in Internal Dosimetry." Health Phys. 41:341-348.

Johnson, J. R., and R. C. Myers. 1981. "Alkaline Earth Metabolism: A Model: Useful in Calculating Organ Burdens, Excretion Rates and Committed Effective Dose Equivalent Conversion Factors." Radiation Protection Dosimetry. $1(2): 87-95$.

Johnson, W. B., E. Shelar, R. E. Ruff, H. B. Singh, and L. Salas. 1975. Gas Tracer Study of Roof-Vent Effluent Diffusion at Millstone Nuclear Power Station. AIF/NESP-007b, Atomic Industrial Forum, Washington, D.C.

Kao, S. K. 1984. "Theories of Atmospheric Transport and Diffusion." Atmospheric Science and Power Production, pp. 109-239. DE84005177 (D0E/TIC-27601), U.S. Department of Energy, Washington, D.C.

Kennedy, W. E., Jr., R. A. Peloquin, B. A. Napier, and S. M. Neuder. 1986. Intruder Dose Pathway Analysis for the Onsite Disposal of Radioactive Wastes: the ONSITE/MAXII Computer Program. NUREG/CR-3620, Supplement No. 1, U. S. Nuclear Regulatory Commission, Washington, D. C.

Kennedy, W. E., Jr., R. A. Peloquin, B. A. Napier, and S. M. Neuder. 1987. Intruder Dose Pathway Analysis for the Onsite Disposal of Radioactive Wastes: the ONSITE/MAXI I Computer Program. NUREG/CR-3620, Supplement No. 2, U. S. Nuclear Regulatory Commission, Washington, D. C.

Kimbrough, R. D., H. Falk, P. Stehr, and G. Fries. 1983. "Health Implications of $2,3,7,8,-$ Tetrachlorodibenzodioxin (TDCC) Contamination of Residential Soil." In Proceedings of Public Health Risks of the Dioxins, October 19-20, 1983. William Kaufmann, Los Angeles, California. 
Kocher, D. C. 1979. Dose-Rate Conversion Factors for External Exposure to Photon and Electron Radiation from Radionuclides Occurring in Routine Releases from Nuclear Fuel Cycle Facilities. NUREG/CR-0494, U. S. Nuclear Regulatory Commission, Washington, D.C.

Kocher, D. C. 1981a. Dose-Rate Conversion Factors for External Exposure to Photons and Electrons. NUREG/CR-1918, U. S. Nuclear Regulatory Commission, Washington, D.C.

Kocher, D. C. 1981b. Radioactive Decay Data Tables: A Handbook of Decay Data for Application to Radiation Dosimetry and Radiological Assessments. DOE/TIC-11026, U. S. Department of Energy, Washington, D.C.

Mansius, C. A. 1969. A Revised Photon Probability Library For Use With ISOSHLD-III. BNWL-236 SUP 2, Pacific Northwest Laboratory, Richland, Washington.

Martin, D. 0., and J. A. Tikvart. 1968. "A General Atmospheric Diffusion Model for Estimating the Effects on Air Quality of One or More Source." Presented at 61st Annual Meeting of the Air Pollution Control Association (APCA), APCA, St. Paul, Minnesota.

McKenzie, D. H., L. L. Cadwe11, K. A. Gano, W. E. Kennedy, Jr., B. A. Napier, R. A. Peloquin, L. A. Prohammer, and M. A. Simmons. 1986. Estimation of Radiation Dose to Man Resulting from Biotic Transport: The Bioport/MAXI1 Software Package. NUREG/CR-2675 Vol. 5, U.S. Nuclear Regulatory Commission, Washington, D.C.

Murty, R. C. 1965. "Effective Atomic Numbers of Heterogeneous Materials." Nature. 207:398-399.

Napier, B. A., R. L. Roswell, W. E. Kennedy, Jr., and D. L. Strenge. 1980. ARRRG and FOOD - Computer Programs for Calculating Radiation Dose to Man from Radionuclides in the Environment. PNL-3180, Pacific Northwest Laboratory, Richland, Washington.

Napier, B. A., W. E. Kennedy, Jr., and J. K. Soldat. 1980. PABLM - A Computer Program to Calculate Accumulated Radiation Dose from Radionuclides in the Environment. PNL-3209, Pacific Nortliwest Laboratory, Richland, Washington.

Napier, B. A., R. A. Peloquin, W. E. Kennedy, Jr., and S. M. Neuder. 1984. Intruder Dose Pathway Analys is for the Onsite Disposal of Radioactive Wastes: The ONSITE/MAXI Computer Prograrn. NUREG/CR-3620, U. S. NuCTear Regulatory Commission, Washington, D.C.

Napier, B. A., R. A. Peloquin, and D. L. Strenge. 1986. DITTY - A Computer Program for Calculating Population Dose Integrated Over Ten Thousand Years. PNL-4456, Pacific Northwest Laboratory, Richland, Washington. 
Nelson, J. L. 1965. "Distribution of Sediments and Associated Radionuclides in the Columbia River Below Hanford." In Hanford Radiological Sciences Research and Development Report for 1964, ed. D. W. Reece and J. K. Green, p. 3.80. USAEC Report BNWL-36, Pacific Northwest Laboratory, Richland, Washington.

O'Brien, K., and R. Sanna. 1976. "The Distribution of Absorbed Dose-Rates in Humans from Exposure to Environmental Gamma Rays." Health Phys. 30:71.

Panofsky, H. A., and J. A. Dutton. 1984. Atmospheric Turbulence. WileyInterscience, New York, New York.

Pinder III, J. E., T. G. Ciravolo, and J. W. Bowling. 1988. "The Interrelationships Among Plant Biomass, Plant Surface Area, and the Interception of Particulate Deposition by Grasses." Health Phys. 55:51-58.

Ramsde11, J. V. 1988. Atmospheric Diffusion for Control Room Habitability Assessments. NUREG/CR-5055, U.S. Nuclear Regulatory Commission, Washington, D.C.

Rockwell III, T., ed. 1956. Reactor Shielding Design Manual. D. Van Nostrand Company, Inc., New York, New York.

Sagendorf, J. F., J. T. Goll, and W. F. Sandusky. 1982. X0QD0Q: Computer Program for the Meteorological Evaluation of Routine Effiuent Releases at Nuclear Power Stations. NUREG/CR-4380, U.S. Nuclear Regulatory Commission, Washington, D.C.

Sehme1, G. A. 1980. "Particle and Gas Deposition: A Review." Atmos. Environment. 14:983-1011.

Schme1, G. A. 1984. "Deposition and Resuspension." Atmospheric Science and Power Production. DOE/TIC-27601, Chapter 12, U.S. Department of Energy, Washington, D.C.

Shor, R. W., C. F. Baes III, and R. D. Sharp. 1982. Agricultural Production in the United States by County: A Compilation of Information from the 1974 Census of Agriculture for Use in Terrestrial Food Chain Transport and Assessment Models. ORNL-5768. Oak Ridge National Laboratory, Oak Ridge, Tennessee.

Simmons, G. L., J. J. Regimbal, J. Greenborg, E. L. Kelley, Jr., H. H. Van Tuy1. 1967. ISOSHLD-II: Code Revision to Include Calculation of Dose Rate from Shielded Bremsstrahlung Sources. BNWL-236 SUP 1, Pacific Northwest Laboratory, Richland, Washington.

Soldat, J. K., N. M. Robinson and D. A. Baker. 1974. Models and Computer Codes for Evaluating Environmental Radiation Doses. BNWL-1754. Battelle, Pacific Northwest Laboratory, Richland, Washington. 
Strenge, D. L. 1975. DACRIN - Modification of Gastrointestinal Tract Dose. BNWL-B-399 SUPP, Pacific Northwest Laboratory, Richland, Washington.

Strenge, D. L., and E. C. Watson. 1973. KRONIC - A Computer Program for Calculating Annual Average External Doses from Chronic Atmospheric Releases of Radionuclides. BNWL-B-264, Pacific Northwest Laboratory, Richland, Washington.

Strenge, D. L., E. C. Watson, and J. R. Houston. 1975. SUBDOSA - A Computer Program for Calculating External Doses from Accidental Atmospheric Releases of Radionuclides. BNWL-B-351, Pacific Northwest Laboratory, Richland, Washington.

Strenge, D. L., R. A. Peloquin, and G. Whelan. 1986. LADTAP II - Technical Reference and User Guide. NUREG/CR-4013, U. S. Nuclear Regulatory Commission, Washington, D.C.

Strenge, D. L., T. J. Bander, and J. K. Soldat. 1987. GASPAR II - Technical Reference and User Guide. NUREG/CR-4653, U.S. Nuclear Regulatory Commission, Washington, D.C.

Tadmor, J., and Y. Gur. 1969. "Analytical Expressions for Vertical and Lateral Dispersion Coefficients in Atmospheric Diffusion." Atmos. Environment. 3:688-689.

Ti11, J. E., and H. R. Meyer. 1983. Radiological Assessment - A Textbook on Environmental Dose Analysis. NUREG/CR-3332, U.S Nuclear Regulatory Commission, Washington, D.C.

Toombs, G. L., and P. B. Cutler, eds. 1963. Comprehensive Final Report for the Lower Columbia River Environmental Survey in Oregon, June 5, 1961 July 31, 1967. Oregon State Board of Health, Division of Sanitation and Engineering, Salem, Oregon.

U.S. Nuclear Regulatory Commission (NRC). 1977a. Calculation of Annual Doses to Man from Routine Releases of Reactor Effluents for the Purpose of Evaluating Compliance with 10 CFR Part 50, Appendix I. Regulatory Guide 1.109 Revision 1, U.S. Nuclear Regulatory Commission, Washington, D.C.

U.S. Nuclear Regulatory Commission (NRC). 1977b. Estimating Aquatic Dispersion of Effluents from Accidental and Routine Reactor Releases for the Purpose of Implementing Appendix I. Regulatory Guide 1.113 Revision 1, U.S. Nuclear Regulatory Commission, Washington, D.C.

U.S. Nuclear Regulatory Commission (NRC). 1977c. Methods for Estimating Atomspheric Transport and Dispersion of Gaseous Effluents in Routine Releases from Light-Water-Cooled Reactors. Regulatory Guide 1.111 Revision 1, U.S. Nuclear Regulatory Commission, Washington, D.C. 
Whicker, F. W., and T. B. Kirchner. 1987. "PATHWAY: A Dynamic Foodchain Model to Predict Radionuclide Ingestion After Fallout Deposition." Health Phys. 52:717-738. 
APPENDIIX

HEDUP SYSTEM DESIGN REQUIREMENTS 

Hanford Environmental Dosimetry Upgrade Project (HEDUP)

TASK 02 -- SYSTEM DESIGN REQUIREMENTS

\section{INTRODUCTION}

Computer codes will be developed on this project for calculating radiation doses to humans from radionuclides released into the environment from Hanford Operations. The computer codes will be used by a number of different groups at Hanford for both retrospective calculations of the radiological impacts of routine Hanford emissions and also for prospective dose calculations, as required for various scenarios. The users would include, among others:

* PNL -- Environmental Evaluations Section, for calculating offsite radiation doses to the general public and the maximally exposed hypothetical individual, as required for preparation of the annual Hanford Environmental Monitoring Report,

-- Environmental and Risk Assessment Section, for use in developing environmental impact statements, safety analysis reports, radiological impacts studies, and environmental assessment work.

* RHO -- Nuclear and Safety Analysis Group, for accidental release analyses and routine release consequence analyses,

-- Regulatory and Safety Review Group, for evaluating the doses resulting from annual average stack releases, reviewing Clean Air Act compliance, and for performance assessment:s,

* HEDL -- Safety Analysis Group, for safety analyses, accident and chronic 
release consequence studies, and environmental assessments,

-- Environmental and Radiological Engineering Group, for safety

analyses, accident and chronic release consequence studies, and environmental assessments,

* UNC -- Radioactive Effluent Control Subsection, for estimating offsite impacts of radioactive releases for environmental assessments, decommissioning activities, and routine and accidental releases.

The computer code developed on this project must therefore be designed to match the needs of multiple users. A committee of representative potential user groups was formed under the coordination of the Hanford Dose Overview Committee to develop recommendations for computer code system design specifications to match the needs of each operating contractor.

This document identifies the system design requirements needed to make the computer codes user-friendly, compatible with current computer facilities, and well-suited to the calculational requirements of individual users.

System design requirements are grouped into the following categories:

1. General computational requirements

2. Computational facilities, hardware, and databases.

3. Code language.

4. Coding Standard and coding standard tools.

5. Input parameters and format:

Release category and source term. Scenarios. 
Meteorology.

Environmental transport.

Exposure pathways.

6. Dosimetry specifications.

7. Risk assessment calculations.

8. Integration of separate codes.

9. Customized pathway requirements.

10. Specialized scenario requirements.

11. Output format.

12. Graphics.

13. Documentation and instructions.

14. Error messages.

15. Updates and revisions.

16. Security.

17. Quality assurance.

18. Training.

These items are discussed in the following sections.

GENERAL COMPUTATIONAL REQUIREMENTS

The capabilities of the computer code shall include, as a minimum, the following:

1. Radiation doses for:

(a) Acute exposure to radionuclides, with option for annual dose, 50-year committed dose, and 70-year committed dose, i.e. $1 / 1,1 / 50,1 / 70$.

(b) Chronic exposure to radionuclides, with option for annual dose, 50-year committed dose, 70-year committed dose, and the 50-year 
cumulative dose and 70-year cumulative dose for a user-specified number of years of exposure, i.e. $1 / 1,1 / 50,1 / 70, x / 50$, and $x / 70$.

2. Exposure pathways to include:

(a) Direct exposure via (1) water (swimming, boating and fishing), (2) soil (surface and buried depositions), (3) air (semi-infinite cloud and the finite plume), and (4) special geometries.

(b) Inhalation pathways, in units of $\mathrm{rem} / \mathrm{Ci}$ and $\mathrm{Sv} / \mathrm{Bq}$ inhaled.

(c) Ingestion pathways, in units of $\mathrm{rem} / \mathrm{Ci}$ and $\mathrm{SV} / \mathrm{Bq}$ ingested.

3. Scenario designs to include:

(a) Acute releases by (1) air at ground level or elevated levels, or by (2) water releases.

(b) Chronic releases by (1) air at ground level or elevated levels, or by (2) water releases.

(c) Initial contamination of (1) soil, (2) buildings and other surfaces, and (3) water.

4. Source term variations to include:

(a) Decay of radionuclides to start of scenario.

(b) Input of total radionuclide inventory or specified fraction.

(c) Input of radionuclide concentrations $\left(\mathrm{Ci} / \mathrm{g}, \mathrm{Ci} / \mathrm{m}^{3}\right.$, etc.)

(d) Release rate of radionuclides to (1) air and (2) water.

5. Interface considerations:

(a) Atmospheric dispersion.

(b) Geohydrology.

(c) Biotic transport.

(d) Surface water transport.

(e) Special shielding calculations.

6. Target population variations by distance and sector: 

(a) Offsite individuals.
(b) Offsite populations.
(c) Onsite individuals.
(d) Intruders.

COMPUTATIONAL FACILITIES, HARDWARE AND DATABASES

The computer code shall be designed for implementation on the UNIVAC-1100 to provide optimum access to the code by users at Hanford contractor facilities. The code shall secondarily be designed for in-house VAX and other minicomputer systems. Design of the code for future application to IBM-PC/AT/XT (or compatible) systems should be considered for the future.

If necessary to improve data processing speeds, provision should be made for the code could to be run on the NAS-9060 LSIS (large scale information system) computer.

The code shall be available to authorized users "on demand." A convenient facility/user interface is desirable for on-line access on a priority basis for general purpose needs, and batch mode for routine work and preparation of the Hanford environmental monitoring report.

\section{CODE LANGUAGE}

The computer code shall be written in ANSI FORTRAN-77 standard language. This choice will facilitate conversion to other computing systems, modifications of the code, and necessary updating. 
CODING STANDARD AND CODING STANDARD TOOLS

Programming shall follow the guidelines of the PNL Statistical Computing Unit FORTRAN coding standard. The coding standard is intended to provide a neat, uniform, and complete form of documentation and quality control, to streamline review efforts by standardizing the format of the code, and to make software maintenance a more simple task.

All FORTRAN routines shall consist of three blocks:

1. Definition block (routine name, limitations and prerequisites, arguments, logical names and files used, routines used),

2. Implementation block (development information, modification history, algorithms, constants, parameter statements, common blocks, and variables), and

3. Code block (data statements and code).

Common blocks, often used constants (parameter statements) and often used variable names shall be handled as stated in their respective sections.

Where applicable, the programmer should localize system-dependent calls, avoid dependency on internal word size (i.e., set up a constant word size), place constants in an up-front parameter file, put variables in Common block, use device names in front of file names, and hold to a limit of one routine per file.

INPUT PARAMETERS AND FORMAT

A user-friendly, menu-driven program will facilitate use of the code by many different Hanford users. An input screen would streamline initial 
data entry and specification of the type of calculation desired.

The option should be added for using a 'runstream' in place of the menu. Runstreams can be quickly copied or altered and used inplace of the menu to initialize a program run.

The code shall organize and display-each input item to allow a user re-check of the data entry.

A namelist input, or the equivalent in standard FORTRAN language should be provided and standardized. The current DACRIN source term input system is much easier to use than the SUBDOSA system. The new code shall allow the user the flexibility of specifying any distance parameters desired.

The maximally exposed individual shall be determined from realistic assumptions concerning location of residence, dietary practices, and living conditions. Unlikely combinations of parameters should be avoided.

Release Category:

Both acute (accidental or one-time) and clironic releases shall be available as options in the calculation. The code shall allow 1) evaluation of acute releases via air submersion, ingestion, and inhalation pathways, and 2) evaluation of chronic releases via air submersion, inhalation, ingestion, and direct exposure (ground contamination, water immersion, etc.) pathways.

Scenarios: 
By allowing the time parameters to be variable, such scenarios as the case of transient residents and intruders could be modeled by the user. The default condition, however, should be for the permanent resident.

\section{Meteorology:}

Three atmospheric dispersion models are needed in the code: 1) the straight-line gaussian model, 2) input tables of $X / Q$, and 3) 95 percentile $X / Q$. These models need to be as complete as possible to keep the results reproducible, standardized, and documented. The code should be able to handle specified distances of from $0.1 \mathrm{~km}$ to $72 \mathrm{~km}$ from the source. The release durations should be variable from as short a time as an instantaneous release, to times as long as one year.

The computer code shall allow simple incorporation of site-specific meteorology. The user shall be allowed to input $X / Q$ values as a number, and the allowed range of $X / Q$ values shall cover the range typical for Hanford conditions. An option shall be provided for ground-level radionuclide releases, and releases from any specified height above ground level. The straight-line model may be appropriate for most applications.

A user-friendly option shall be provided for the finite cloud model for both elevated releases and ground-level releases.

It would be useful if the code also took into account plume depletion and deposition from the plume to ground.

The PNL Atmospheric group should be involved in the preparation of the 
meteorological aspects of the code.

Environmental Transport:

The code shall take into account the effect of long-term weathering of radionuclides in soil, i.e. dispersion irito the below-surface soil matrix.

\section{Exposure Pathways:}

Deposition on soil shall be considered for both atmospheric deposition and irrigation using contaminated water.

The uptake of radionuclides in the soil by plants shall be accounted for using current methods. The typical user will not have input data for this pathway, and must rely on the best available models, concentration ratios, or measured concentrations as currently recommended.

The uptake of radionuclides by farm animals shall take into account the ingestion of crops and the consumption of contaminated water.

Commonly used or typical exposure pathway parameters shall be established in the code as default values.

\section{DOSIMETRY SPECIFICATIONS}

GENMOD shall replace DACRIN; however, some of the valuable input/output features of DACRIN shall be retained. Internal dosimetry calculation shall be based on ICRP-30 models and biokinetic values for radionuclide residency and transport in the body. 
GENMOD incorporates additional models other than those of the ICRP. These shall be available at the option of the user. These models include the alkaline earth model, the MIRD iron model, the ${ }^{14} \mathrm{C}$ model, etc.

Provision shall be made for revising biokinetic models and input parameters as updated values become available.

The code should permit the user the option of inserting desired input parameters (e.g. breathing rate, quantity of water or foodstuffs consumed), although such usage should be considered nonstandard and so identified to the user. Standard default values should be easily recognized.

The code shall calculate the effective dose equivalent for the average member of the general public in the sector and distance of interest, as well as for the maximally exposed individual.

The code shall provide integration of doses for any time period of choice following radionuclide release. This choice should be an input parameter.

The user shall be able to set up the dose calculation to provide the annual (one year) dose and dose equivalent, the 50-year committed dose equivalent, and cumulative doses expected from both acute and chronic exposures from ground level and elevated releases, as well as liquid releases to river and drinking water pathways.

The calculation shall account for radionuclide decay "in transit, " i.e., the diminishing activity of radionuclides between time of release and time 
of exposure of the target individual.

DACRIN currently has the problem that erroneous results appear when dosimetry is requested for more than seven organs. The new code should permit the user to obtain dose data for all organs, including the skin.

Standard default values shall be given for breathing rates assumed for the exposed population, with the user option to change the values. Onsite occupancy factors shall be considered for the calculation of dose to the maximally exposed onsite individual.

The code shall allow the user to specify the activity median aerodynamic diameter of the inhaled aerosol for cases involving inhalation of radionuclides. The code shall calculate deposition fractions in the three major lung regions for the particle size selected, instead of prompting the user to supply those deposition fractions.

GENMOD currently provides output for Class $D, W$, or $Y$ materials. Often, however, the radioactive aerosol may contain a mixture of Class $D, W$, and $Y$ materials. GENMOD shall be modifted to consider mixtures or combinations of different solubility classes, and produce output for that mixture.

In the foodchain pathway, an option shall be provided to specify the total crop production versus the number of people consuming those crops.

The population dose may be estimated from the average dose to the average resident times the number of persons involved. Doses shall therefore be 
calculated for individuals and then multiplied by the number of persons.

Age considerations should not be necessary at this time, but provision should be made for future incorporation of age-dependent models.

\section{RISK ASSESSMENT CALCULATIONS}

The code shall calculate the 50-year and 70-year committed effective dose equivalent for individuals, and the 50-year and 70-year effective dose equivalent commitment (i.e. the collective effective dose equivalent commitment) for populations.

\section{INTEGRATION OF SEPARATE CODES}

The final product will be a single code developed from several existing codes. This code shall carefully integrate all components to facilitate ease of program execution.

\section{CUSTOMIZED PATHWAY REQUIREMENTS}

None are identified at this time. Should the need arise, they could be added to the code, and provision should be made accordingly.

\section{SPECIALIZED SCENARIO REQUIREMENTS}

The option to allow input of externally-calculated meteorology data would cover any specialized scenarios, including release of radionuclides during postulated tornado conditions.

\section{OUTPUT FORMAT}

At the beginning of the report output, the input variables shall be repeated 
back for user verification.

The output shall be either in terms of (1) traditional quantities and units, such as rem, millirem, curies, etc., or (2) S.I. units, such as sievert, millisievert, becquerel, etc., at the option of the user.

The output format shall include consequences at any given input distance rather than requiring interpolation within a set group of distances (as currently the case with certain modes in SUBDOSA). The output shall match the desired input.

A summary of the doses calculated shall be provided for each contributing pathway and organ. The summary shall also include a listing of the major contributing radionuclides, and the percent of the total dose for each pathway contributed by each radionuclide.

Literature references to the published sources on which the computer code is based shall be incorporated into the program and should be callable, with printout capability, at the option of the user.

\section{GRAPHICS}

There are no graphics needs currently identified. However, it is expected that there will be a future need to graphically display numbers calculated by the computer code. An example might be a plot of $X / Q$ versus distance from the point of release. A mechanism shall be designed therefore, to dump output into files for later graphics applications. 
DOCUMENTATION AND INSTRUCTIONS

The user's manual shall be a living document that is updated whenever a change is made. User's manuals shall be maintained in three-ring binders that are easily updated. Numbered copies shall be distributed to individuals on a list of users.

\title{
ERROR MESSAGES
}

Error messages shall assist the user when improper input parameters or formats are used. Error messages should not occur when the data is properly entered.

\begin{abstract}
All input errors shall be identified by the input routines prior to program execution.
\end{abstract}

UPDATES AND REVISIONS

Notices of code updates, and revised instructions shall be provided to potential users. A list of potential users shall be maintained so that revisions and notices may be distributed, as appropriate.

\section{SECURITY}

The computer code and associated databases are to be considered sensitive. Security measures shall be implemented to protect the code from access by unathorized users.

Configuration control shall be a feature of the software to protect the basic code from unauthorized changes. A control mechanism with sign-off procedures shall be implemented to protect the software from unauthorized 
modifications. Needed changes shall be validated before modification are permitted (see Quality Assurance).

\section{QUALITY ASSURANCE}

PNL's Procedures for License-related Programs for quality assurance shall be followed. Software control procedures are given in SCP-301 through SCP 312, and include:

-- magnetic media protection and control,

-- software development specification preparation and approval,

-- computer code verification and/or validation,

-- computer code configuration control,

-- computer code acceptance testing,

-- computer code and documentation change control,

-- computer code applịcation control,

-- final internal development review of computer code and documentation,

-- computer software transfer,

-- utility code documentation, control, testing and use,

-- documenting and reporting discrepancies found during computer code control, testing and use, and

-- determination of software classification.

\section{TRAINING}

A short training program shall be developed at the completion of the code to instruct potential users on the execution of the code. A detailed stepwise instruction manual shall also be prepared. Training should consist of class sessions and hand-out instructions, with copportunity for hands-on testing of the code. 
